Syracuse University

SURFACE at Syracuse University

\title{
Asymptotic Properties of Estimators for the Linear Panel Regression Model with Individual Effects and Serially Correlated Errors: The Case of Stationary and Non-Stationary Regressors and Residuals
}

\author{
Badi H. Baltagi \\ Syracuse University, bbaltagi@maxwell.syr.edu \\ Chihwa Kao \\ Syracuse University, cdkao@maxwell.syr.edu \\ Long Liu \\ Syracuse University
}

Follow this and additional works at: https://surface.syr.edu/cpr

Part of the Mathematics Commons

\section{Recommended Citation}

Baltagi, Badi H.; Kao, Chihwa; and Liu, Long, "Asymptotic Properties of Estimators for the Linear Panel Regression Model with Individual Effects and Serially Correlated Errors: The Case of Stationary and NonStationary Regressors and Residuals" (2007). Center for Policy Research. 72.

https://surface.syr.edu/cpr/72

This Working Paper is brought to you for free and open access by the Maxwell School of Citizenship and Public Affairs at SURFACE at Syracuse University. It has been accepted for inclusion in Center for Policy Research by an authorized administrator of SURFACE at Syracuse University. For more information, please contact surface@syr.edu. 


\title{
Center for Policy Research Working Paper No. 93
}

\author{
ASYMPTOTIC PROPERTIES OF ESTIMATORS FOR \\ THE LINEAR PANEL REGRESSION MODEL WITH \\ INDIVIDUAL EFFECTS AND SERIALLY CORRELATED \\ ERRORS: THE CASE OF STATIONARY AND \\ NON-STATIONARY REGRESSORS AND RESIDUALS \\ Badi H. Baltagi, Chihwa Kao, and Long Liu
}

\author{
Center for Policy Research \\ Maxwell School of Citizenship and Public Affairs \\ Syracuse University \\ 426 Eggers Hall \\ Syracuse, New York 13244-1020 \\ (315) 443-3114 | Fax (315) 443-1081 \\ e-mail: ctrpol@syr.edu
}

April 2007

$\$ 5.00$

Up-to-date information about CPR's research projects and other activities is available from our World Wide Web site at www-cpr.maxwell.syr.edu. All recent working papers and Policy Briefs can be read and/or printed from there as well. 


\title{
CENTER FOR POLICY RESEARCH - Spring 2007
}

\author{
Timothy Smeeding, Director \\ Professor of Economics \& Public Administration
}

\section{Associate Directors}

\author{
Margaret Austin \\ Associate Director \\ Budget and Administration
}

\author{
Douglas Wolf \\ Professor of Public Administration \\ Associate Director, Aging Studies Program
}

\begin{abstract}
John Yinger
Professor of Economics and Public Administration Associate Director, Metropolitan Studies Program
\end{abstract}

\section{SENIOR RESEARCH ASSOCIATES}

Badi Baltagi

Pablo Beramendi......

Dan Black.

William Duncombe

Gary Engelhardt

Deborah Freund

Madonna Harrington Meyer

Christine Himes

William C. Horrace

Duke Kao

Eric Kingson

Thomas Kniesner

Jeffrey Kubik

Andrew London
Economics

Political Science

.. Economics

Public Administration Economics

Public Administration Sociology Sociology

Economics

Economics

Social Work

Economics

Economics Sociology
Len Lopoo

Jerry Miner

Jan Ondrich

John Palmer

Lori Ploutz-Snyder.

David Popp.

Grant Reeher

Christopher Rohlfs

Stuart Rosenthal

Ross Rubenstein

Margaret Usdansky

Michael Wasylenko

Janet Wilmoth
Public Administration Economics Economics Public Administration Exercise Science

Public Administration Political Science ..Economics Economics Public Administration ..Sociology Economics Sociology

\section{GRADUATE ASSOCIATES}

Javier Baez

Sonali Ballal Jesse Bricker

Maria Brown

Mike Eriksen

Qu Feng

Katie Fitzpatrick

Alexandre Genest

Julie Anna Golebiewski

Nadia Greenhalgh-Stanley

Tamara Hafner

Yue $\mathrm{Hu}$
Economics

Public Administration

Economics

Social Science

Economics

Economics

Economics

Public Administration

Economics

Economics

Public Administration

Economics
Sung Hyo Hong

Joseph Marchand....

Neelakshi Medhi

Larry Miller

Wendy Parker

Emily Pas ....

Shawn Rohlin

Cynthia Searcy

Jeff Thompson

Coady Wing

Daniel Yanulavich

Ryan Yeung
Economics

Economics

Social Science

Public Administration Sociology

Economics Economics

Public Administration Economics

Public Administration

Public Administration

Public Administration

\section{STAFF}

Kelly Bogart Administrative Secretary Martha Bonney... ...Publications/Events Coordinator Karen Cimilluca...............Administrative Secretary Kitty Nasto.
Candi Patterson. Computer Consultant Mary Santy..... Administrative Secretary

Tammy Tanner Librarian/Office Coordinator 


\section{Abstract}

This paper studies the asymptotic properties of standard panel data estimators in a simple panel regression model with error component disturbances. Both the regressor and the remainder disturbance term are assumed to be autoregressive and possibly non-stationary. Asymptotic distributions are derived for the standard panel data estimators including ordinary least squares, fixed effects, first-difference, and generalized least squares (GLS) estimators when both $\mathrm{T}$ and $\mathrm{n}$ are large. We show that all the estimators have asymptotic normal distributions and have different convergence rates dependent on the non-stationarity of the regressors and the remainder disturbances. We show using Monte Carlo experiments that the loss in efficiency of the OLS, FE and FD estimators relative to true GLS can be substantial.

Key Words: Panel Data, OLS, Fixed-Effects, First-Difference, GLS

JEL classification: C33 


\title{
Asymptotic Properties of Estimators for the Linear Panel Regression Model with Individual effects and Serially Correlated Errors: The Case of Stationary and Non-Stationary Regressors and Residuals
}

\author{
Badi H. Baltagi*, Chihwa Kao†, Long Liu \\ Syracuse University
}

April 22, 2007

\begin{abstract}
This paper studies the asymptotic properties of standard panel data estimators in a simple panel regression model with error component disturbances. Both the regressor and the remainder disturbance term are assumed to be autoregressive and possibly non-stationary. Asymptotic distributions are derived for the standard panel data estimators including ordinary least squares, fixed effects, first-difference, and generalized least squares (GLS) estimators when both $T$ and $n$ are large. We show that all the estimators have asymptotic normal distributions and have different convergence rates dependent on the non-stationarity of the regressors and the remainder disturbances. We show using Monte Carlo experiments that the loss in efficiency of the OLS, FE and FD estimators relative to true GLS can be substantial.
\end{abstract}

Key Words: Panel Data, OLS, Fixed-Effects, First-Difference, GLS.

\section{Introduction}

Econometricians have long been concerned with conditions under which the ordinary least squares (OLS) estimator is asymptotically efficient. The standard textbook result is that, under a general variance-covariance

*Address correspondence to: Badi H. Baltagi, Center for Policy Research, 426 Eggers Hall, Syracuse University, Syracuse, NY 13244-1020; e-mail: bbaltagi@maxwell.syr.edu.

${ }^{\dagger}$ Chihwa Kao, Center for Policy Research, 426 Eggers Hall, Syracuse University, Syracuse, NY 13244-1020; e-mail: cdkao@maxwell.syr.edu.

$\ddagger$ Long Liu, Economics Department, 110 Eggers Hall, Syracuse University, Syracuse, NY 13244-1020; e-mail: loliu@maxwell.syr.edu. 
structure on the disturbances, the OLS estimator is less efficient than generalized least squares (GLS). This is well documented for the case of stationary autoregressive disturbances and stationary regressors. However, Phillips and Park (1988) showed that in a regression with integrated regressors, OLS and GLS are asymptotically equivalent.

Recently, Choi (1999) studied the limiting distributions of the fixed effects (FE), GLS, and within-GLS estimators for a panel data regression model with autoregressive disturbances, while Choi (2002) extended this work to instrumental variables (IV) estimation. Phillips and Moon (1999) presented a fundamental framework for studying sequential and joint limit theories in nonstationary panel data analysis, while Kao (1999) studied the asymptotic properties of the FE estimator of a spurious regression and proposed residualbased tests for panel cointegration. See Baltagi and Kao (2000), Choi (2006) and Breitung and Pesaran (2006) for recent surveys of this rapidly growing subject. In an early finding, Baltagi and Krämer (1997) showed the equivalence of the GLS and FE estimators in a simple panel data regression with time trend as a regressor. Kao and Emerson (2004a, 2004b) extended Baltagi and Krämer to a model with serially correlated remainder errors. Kao and Emerson showed that the FE estimator is asymptotically equivalent to GLS when the error term is $I(0)$; but that GLS is more efficient than FE when the error term is $I(1)$. It is known that the panel time trend can be seen as a special case of the panel regression with a non-zero drift $I(1)$ regressor.

This paper extends the literature by studying the asymptotic properties of OLS, FE, first difference (FD) and GLS in the panel regression with an autocorrelated regressor and an autocorrelated remainder error (both of which can be stationary or nonstationary). We show that when the error term is $I(0)$ and the regressor is $I(1)$, the FE estimator is asymptotically equivalent to the GLS estimator and OLS is less efficient than GLS (due to a slower convergence speed). However, when the error term and the regressor are $I(1)$, GLS is more efficient than the FE estimator since GLS is $\sqrt{n T}$ consistent, while FE is $\sqrt{n}$ consistent. This implies that GLS is the preferred estimator under both cases (i.e., regression error is either $I(0)$ or $I(1))$.

All asymptotic results in this paper assume that $T \rightarrow \infty$ followed by $n \rightarrow \infty$. We use $(n, T) \stackrel{\text { seq }}{\rightarrow} \infty$ to denote this sequential limit. We write the integral $\int_{0}^{1} W(s) d s$ as $\int W$ and $\tilde{W}$ as $W-\int W$ when there is no ambiguity over limits. $\Rightarrow$ to denote weak convergence, $\equiv$ to denote equivalence in distribution, $\stackrel{p}{\rightarrow}$ to denote convergence in probability, $[x]$ to denote the largest integer $\leq x, I(0)$ and $I(1)$ to signify a time series that is integrated of order zero and one, respectively, and $B M(\Omega)$ to denote Brownian motion with covariance matrix $\Omega$. All proofs are collected in an appendix available upon request from the authors. 


\section{The Model and Assumptions}

Consider the following panel regression:

$$
y_{i t}=\alpha+x_{i t} \beta+u_{i t}, \quad i=1, \ldots, n, \quad t=1, \ldots, T
$$

where $u_{i t}=\mu_{i}+\nu_{i t}$, and $\alpha$ and $\beta$ are scalars. For simplicity, we consider the case of one regressor, but our results can be extended to the multiple regressor case. We assume that $\mu_{i} \sim i i d\left(0, \sigma_{\mu}^{2}\right)$ and $\left\{\nu_{i t}\right\}$ is $\operatorname{AR}(1)$

$$
\nu_{i t}=\rho \nu_{i t-1}+e_{i t}, \quad|\rho| \leq 1
$$

where $e_{i t}$ is a white noise process with variance $\varpi_{e}^{2}$.

Let $x_{i t}$ be also an $\mathrm{AR}(1)$ such that

$$
x_{i t}=\lambda x_{i t-1}+\varepsilon_{i t}, \quad|\lambda| \leq 1
$$

where $\varepsilon_{i t}$ is a white noise process with variance $\varpi_{\varepsilon}^{2}$. In this paper, we assume that

$$
E\left(\mu_{i} \mid x_{i t}\right)=0 .
$$

The initialization of this system is $y_{i 1}=x_{i 1}=O_{p}(1)$ for all $i$. Our interest is in the estimation of the common slope $\beta$. This paper shows that the asymptotic properties of OLS, FE, FD, and GLS estimators depend crucially on the serial correlation properties of $x_{i t}$ and $v_{i t}$. When $y_{i t}$ and $x_{i t}$ are both $I(1)$ but $v_{i t}$ is $I(0)$, equation (1) is a panel cointegrated model. On the other hand, when $v_{i t}$ is $I(1)$ and $y_{i t}$ and $x_{i t}$ are both $I(1)$, equation (1) is a panel spurious model. FE estimators for panel cointegrated and panel spurious models have been discussed in Phillips and Moon (1999) and Kao (1999). The case of a panel time trend model, $x_{i t}=t$, has been studied by Baltagi and Krämer (1997) and Kao and Emerson (2004a, 2004b).

Next, we characterize the innovation vector $\mathbf{w}_{i t}=\left(e_{i t}, \varepsilon_{i t}\right)^{\prime}$. We assume that $\mathbf{w}_{i t}$ is a linear process that satisfies the following assumption:

Assumption 1 For each $i$, we assume:

1. $\mathbf{w}_{i t}=\Pi(L) \boldsymbol{\eta}_{i t}=\sum_{j=0}^{\infty} \Pi_{j} \boldsymbol{\eta}_{i t-j}, \sum_{j=0}^{\infty} j^{a}\left\|\Pi_{j}\right\|<\infty,|\Pi(1)| \neq 0$ for some $a>1$.

2. For a given $i, \boldsymbol{\eta}_{i t}$ is i.i.d. with zero mean and variance-covariance matrix $\Xi$, and finite fourth order cumulants.

Assumption 2 We assume $\boldsymbol{\eta}_{i t}$ and $\boldsymbol{\eta}_{j t}$ are independent for $i \neq j$. That is we assume cross-sectional independence for our model. 
Assumption 1 implies that the partial sum process $\frac{1}{\sqrt{T}} \sum_{t=1}^{[T r]} \mathbf{w}_{i t}$ satisfies the following multivariate invariance principle:

$$
\frac{1}{\sqrt{T}} \sum_{t=1}^{[T r]} \mathbf{w}_{i t} \Rightarrow \mathbf{B}_{i}(r)=\mathbf{B M}_{i}(\Omega) \text { as } T \rightarrow \infty \text { for all } i
$$

where

$$
\mathbf{B}_{i}=\left[\begin{array}{c}
B_{e i} \\
B_{\varepsilon i}
\end{array}\right]
$$

The long-run $2 \times 2$ covariance matrix of $\left\{\mathbf{w}_{i t}\right\}$ is given by

$$
\begin{aligned}
\Omega & =\sum_{j=-\infty}^{\infty} E\left(\mathbf{w}_{i j} \mathbf{w}_{i 0}^{\prime}\right) \\
& =\Pi(1) \Xi \Pi(1)^{\prime} \\
& =\left[\begin{array}{cc}
\varpi_{e}^{2} & \varpi_{e \varepsilon} \\
\varpi_{e \varepsilon} & \varpi_{\varepsilon}^{2}
\end{array}\right] .
\end{aligned}
$$

The long-run covariance matrix can be decomposed into $\Omega=\Sigma+2 \Gamma$, where

$$
\Gamma=\sum_{j=1}^{\infty} E\left(\mathbf{w}_{i j} \mathbf{w}_{i 0}^{\prime}\right)=\left[\begin{array}{cc}
\gamma_{e}^{2} & \gamma_{e \varepsilon} \\
\gamma_{e \varepsilon} & \gamma_{\varepsilon}^{2}
\end{array}\right]
$$

and

$$
\Sigma=E\left(\mathbf{w}_{i 0} \mathbf{w}_{i 0}^{\prime}\right)=\left[\begin{array}{cc}
\sigma_{e}^{2} & \sigma_{e \varepsilon} \\
\sigma_{e \varepsilon} & \sigma_{\varepsilon}^{2}
\end{array}\right] .
$$

Assuming $\varpi_{\varepsilon}^{2}$ is non-zero, we define

$$
\varpi_{e . \varepsilon}=\varpi_{e}^{2}-\frac{\varpi_{e \varepsilon}^{2}}{\varpi_{\varepsilon}^{2}}
$$

Then, $B_{i}$ can be rewritten as

$$
\mathbf{B}_{i}=\left[\begin{array}{c}
B_{e i} \\
B_{\varepsilon i}
\end{array}\right]=\left[\begin{array}{cc}
\varpi_{e . \varepsilon} & \varpi_{e \varepsilon} / \varpi_{\varepsilon} \\
0 & \varpi_{\varepsilon}
\end{array}\right]\left[\begin{array}{c}
V_{i} \\
W_{i}
\end{array}\right]
$$

where $\left[\begin{array}{c}V_{i} \\ W_{i}\end{array}\right]=\mathbf{B M}(I)$ is a standardized Brownian motion. Define the one-sided long-run covariance

$$
\begin{aligned}
\Delta & =\Sigma+\Gamma \\
& =\sum_{j=0}^{\infty} E\left(\mathbf{w}_{i j} \mathbf{w}_{i 0}^{\prime}\right)
\end{aligned}
$$

with

$$
\Delta=\left[\begin{array}{ll}
\delta_{e}^{2} & \delta_{e \varepsilon} \\
\delta_{e \varepsilon} & \delta_{\varepsilon}^{2}
\end{array}\right]
$$


The assumption of constant variances/covariances across $i$, such as in $\Omega, \Sigma$, and $\Gamma$ is used to simplify the notation. It can be extended into the case where different variances are allowed for different $i$ at the expense of more complicated notation.

\section{OLS Estimator}

The OLS estimator of $\beta$ is given by

$$
\widehat{\beta}_{O L S}=\frac{\sum_{i=1}^{n} \sum_{t=1}^{T}\left(x_{i t}-\overline{\bar{x}}\right)\left(y_{i t}-\overline{\bar{y}}\right)}{\sum_{i=1}^{n} \sum_{t=1}^{T}\left(x_{i t}-\overline{\bar{x}}\right)^{2}}
$$

where $\overline{\bar{x}}=\frac{1}{n T} \sum_{i=1}^{n} \sum_{t=1}^{T} x_{i t}$ and $\overline{\bar{y}}=\frac{1}{n T} \sum_{i=1}^{n} \sum_{t=1}^{T} y_{i t}$.

Theorem 1 Under Assumptions 1 - 2, we obtain the following results:

1. If $|\rho|<1$ and $|\lambda|<1$,

(a) $\widehat{\beta}_{O L S}-\beta \stackrel{p}{\rightarrow} \frac{1-\lambda^{2}}{\sigma_{\varepsilon}^{2}}\left[\lim \frac{1}{n T} \sum_{i=1}^{n} \sum_{t=1}^{T} E\left(x_{i t} \nu_{i t}\right)\right]$,

(b) $\sqrt{n T}\left(\widehat{\beta}_{O L S}-\beta-\tau_{1 N T}^{O L S}\right) \Rightarrow N\left(0, \kappa_{1}^{O L S}\right)$,

where $\tau_{1 N T}^{O L S}=\frac{\lim \frac{1}{n T} \sum_{i=1}^{n} \sum_{t=1}^{T} E\left(x_{i t} \nu_{i t}\right)}{\frac{1}{n T} \sum_{i=1}^{n} \sum_{t=1}^{T}\left(x_{i t}-\overline{\bar{x}}\right)^{2}}, \kappa_{1}^{O L S}=\frac{1}{\sigma_{\varepsilon}^{4}}\left[\sigma_{\mu}^{2} \varpi_{\varepsilon}^{2}+\frac{\left(1-\lambda^{2}\right)^{2}}{(1-\rho \lambda)^{2}}\left[\psi_{00}+\sum_{r=1}^{\infty} \lambda^{2 r} \psi_{0 r}+\sum_{r=1}^{\infty} \rho^{2 r} \psi_{r 0}\right]\right]$, $\psi_{0 r}=E\left(\varepsilon_{i(t-r)}^{2} e_{i t}^{2}\right), \psi_{r 0}=E\left(\varepsilon_{i t}^{2} e_{i(t-r)}^{2}\right)$, and $\psi_{00}=E\left(\varepsilon_{i t}^{2} e_{i t}^{2}\right)$.

2. If $\rho=1$ and $|\lambda|<1$,

(a) $\widehat{\beta}_{O L S}-\beta \stackrel{p}{\rightarrow} \frac{(1+\lambda)\left(-\frac{1}{2} \varpi_{e \varepsilon}+\delta_{e \varepsilon}\right)}{\sigma_{\varepsilon}^{2}}$

(b) $\sqrt{n}\left(\widehat{\beta}_{O L S}-\beta-\tau_{2 n T}^{O L S}\right) \Rightarrow N\left(0, \kappa_{2}^{O L S}\right)$,

where $\tau_{2 n T}^{O L S}=\frac{\left(\frac{1}{n} \sum_{i=1}^{n} \frac{1}{T} \sum_{t=1}^{T} \nu_{i(t-1)} e_{i t}\right) \frac{\varpi_{e \varepsilon}}{\varpi^{2}}+\delta_{e \varepsilon}}{(1-\lambda) \frac{1}{n T} \sum_{i=1}^{n} \sum_{t=1}^{T}\left(x_{i t}-\overline{\bar{x}}\right)^{2}}, \kappa_{2}^{O L S}=\frac{(1+\lambda)^{2} \varpi_{\varepsilon . e} \varpi_{e}^{2}}{2 \sigma_{\varepsilon}^{4}}$.

3. If $|\rho|<1$ and $\lambda=1$,

(a) $\sqrt{T}\left(\widehat{\beta}_{O L S}-\beta\right) \stackrel{p}{\rightarrow} 0$,

(b) $\sqrt{n T}\left(\widehat{\beta}_{O L S}-\beta\right) \Rightarrow N\left(0, \kappa_{3}^{O L S}\right)$,

where $\kappa_{3}^{O L S}=\frac{4 \sigma_{\mu}^{2}}{3 \varpi_{\varepsilon}^{2}}$.

4. If $\rho=1$ and $\lambda=1$, 
(a) $\widehat{\beta}_{O L S}-\beta \stackrel{p}{\rightarrow} \frac{2 \delta_{\varepsilon e}}{\varpi_{\varepsilon}^{2}}$,

(b) $\sqrt{n}\left(\widehat{\beta}_{O L S}-\beta-\tau_{4 N T}^{O L S}\right) \Rightarrow N\left(0, \kappa_{4}^{O L S}\right)$,

where $\tau_{4 n T}^{O L S}=\frac{\left(\frac{1}{n} \sum_{i=1}^{n} \frac{1}{T} \sum_{t=1}^{T} x_{i(t-1)} \varepsilon_{i t}\right) \frac{\varpi_{\varepsilon e}}{\varpi^{2}}+\delta_{\varepsilon e}}{\frac{1}{n} \sum_{i=1}^{n} \frac{1}{T^{2}} \sum_{t=1}^{T}\left(x_{i t}-\bar{x}_{i}\right)^{2}}, \kappa_{4}^{O L S}=\frac{2 \varpi_{e \cdot \varepsilon}}{3 \varpi_{\varepsilon}^{2}}$.

It is important to note that $\varpi_{e . \varepsilon} / \varpi_{\varepsilon}^{2}$ can be seen as the long-run signal-to-noise ratio. The OLS estimator ignores the individual effects in the disturbance term. Thus, the variance of $\mu_{i}$, i.e., $\sigma_{\mu}^{2}$ might appear in the variance-covariance matrix of $\widehat{\beta}_{O L S}$ depending on the case considered. In case 1 , both $\mu_{i}$ and $\nu_{i t}$ affect the variance of $\widehat{\beta}_{O L S}$. In cases 2 and $4, \nu_{i t}$ dominates $\mu_{i}$. In case $3, \mu_{i}$ dominates $\nu_{i t}$ and hence the convergence speed is $\sqrt{n T}$, which differs from the $T$-asymptotics in the panel cointegration literature. Also the asymptotic normality of the OLS estimator comes naturally. When summing across $i$, the nonstandard asymptotic distribution due to unit root in the time dimension, such as for cases $2-4$, is smoothed out.

Corollary 1 When $E\left(e_{i t} \varepsilon_{i(t+k)}\right)=0$ for all $i$ and $k$, under the assumptions in Theorem 1, then

1. If $|\rho|<1$ and $|\lambda|<1$,

$$
\sqrt{n T}\left(\widehat{\beta}_{O L S}-\beta\right) \Rightarrow N\left(0, \frac{\sigma_{\mu}^{2}}{\sigma_{\varepsilon}^{2}}+\frac{\left(1-\lambda^{2}\right)^{2}\left[\psi_{00}+\sum_{r=1}^{\infty} \lambda^{2 r} \psi_{0 r}+\sum_{r=1}^{\infty} \rho^{2 r} \psi_{r 0}\right]}{(1-\rho \lambda)^{2} \sigma_{\varepsilon}^{4}}\right) .
$$

When $\varepsilon_{i t}$ and $e_{i t}$ are independent, $\sqrt{n T}\left(\widehat{\beta}_{O L S}-\beta\right) \Rightarrow N\left(0, \frac{\sigma_{\mu}^{2}}{\sigma_{\varepsilon}^{2}}+\frac{(1+\rho \lambda)\left(1-\lambda^{2}\right) \sigma_{e}^{2}}{(1-\rho \lambda)\left(1-\rho^{2}\right) \sigma_{\varepsilon}^{2}}\right)$.

2. If $\rho=1$ and $|\lambda|<1$,

$$
\sqrt{n}\left(\widehat{\beta}_{O L S}-\beta\right) \Rightarrow N\left(0, \frac{(1-\lambda)^{2} \sigma_{e}^{2}}{2 \sigma_{\varepsilon}^{2}}\right)
$$

3. If $|\rho|<1$ and $\lambda=1$,

$$
\sqrt{n T}\left(\widehat{\beta}_{O L S}-\beta\right) \Rightarrow N\left(0, \frac{4 \sigma_{\mu}^{2}}{3 \sigma_{\varepsilon}^{2}}\right)
$$

4. If $\rho=1$ and $\lambda=1$,

$$
\sqrt{n}\left(\widehat{\beta}_{O L S}-\beta\right) \Rightarrow N\left(0, \frac{2 \sigma_{e}^{2}}{3 \sigma_{\varepsilon}^{2}}\right) .
$$

Corollary 1 follows directly from Theorem 1 .

\section{FE Estimator}

The Fixed-Effects estimator of $\beta$ is given by

$$
\widehat{\beta}_{F E}=\frac{\sum_{i=1}^{n} \sum_{t=1}^{T}\left(x_{i t}-\bar{x}_{i}\right)\left(y_{i t}-\bar{y}_{i}\right)}{\sum_{i=1}^{n} \sum_{t=1}^{T}\left(x_{i t}-\bar{x}_{i}\right)^{2}},
$$

where $\bar{x}_{i}=\frac{1}{T} \sum_{t=1}^{T} x_{i t}$ and $\bar{y}_{i}=\frac{1}{T} \sum_{t=1}^{T} y_{i t}$. 
Theorem 2 Under Assumptions 1 - 2, we have the following results:

1. If $|\rho|<1$ and $|\lambda|<1$,

(a) $\widehat{\beta}_{F E}-\beta \stackrel{p}{\rightarrow} \frac{1-\lambda^{2}}{\sigma_{\varepsilon}^{2}}\left[\lim \frac{1}{n T} \sum_{i=1}^{n} \sum_{t=1}^{T} E\left(x_{i t} \nu_{i t}\right)\right]$,

(b) $\sqrt{n T}\left(\widehat{\beta}_{F E}-\beta-\tau_{1 n T}^{F E}\right) \Rightarrow N\left(0, \kappa_{1}^{F E}\right)$,

where $\tau_{1 n T}^{F E}=\frac{\lim \frac{1}{n T} \sum_{i=1}^{n} \sum_{t=1}^{T} E\left(x_{i t} \nu_{i t}\right)}{\frac{1}{n} \sum_{i=1}^{n} \frac{1}{T} \sum_{t=1}^{T}\left(x_{i t}-\bar{x}_{i}\right)^{2}}, \kappa_{1}^{F E}=\frac{\left(1-\lambda^{2}\right)^{2}\left[\psi_{00}+\sum_{r=1}^{\infty} \lambda^{2 r} \psi_{0 r}+\sum_{r=1}^{\infty} \rho^{2 r} \psi_{r 0}\right]}{(1-\rho \lambda)^{2} \sigma_{\varepsilon}^{4}}, \psi_{0 r}=E\left(\varepsilon_{i(t-r)}^{2} e_{i t}^{2}\right)$, $\psi_{r 0}=E\left(\varepsilon_{i t}^{2} e_{i(t-r)}^{2}\right)$, and $\psi_{00}=E\left(\varepsilon_{i t}^{2} e_{i t}^{2}\right)$.

2. If $\rho=1$ and $|\lambda|<1$,

(a) $\widehat{\beta}_{F E}-\beta \stackrel{p}{\rightarrow} \frac{(1+\lambda)\left(-\frac{1}{2} \varpi_{e \varepsilon}+\delta_{e \varepsilon}\right)}{\sigma_{\varepsilon}^{2}}$,

(b) $\sqrt{n}\left(\widehat{\beta}_{F E}-\beta-\tau_{2 n T}^{F E}\right) \Rightarrow N\left(0, \kappa_{2}^{F E}\right)$,

where $\tau_{2 n T}^{F E}=\frac{\left(\frac{1}{n} \sum_{i=1}^{n} \frac{1}{T} \sum_{t=1}^{T}\left(\nu_{i t}-\bar{\nu}_{i}\right) e_{i t}\right) \frac{\varpi_{e \varepsilon}}{\varpi_{e}^{2}}+\delta_{e \varepsilon}}{(1-\lambda) \frac{1}{n} \sum_{i=1}^{n} \frac{1}{T^{2}} \sum_{t=1}^{T}\left(x_{i t}-\bar{x}_{i}\right)^{2}}$ and $\kappa_{2}^{F E}=\frac{(1-\lambda)^{2} \varpi_{\varepsilon} \varpi_{e}^{2}}{6 \sigma_{\varepsilon}^{4}}$.

3. If $|\rho|<1$ and $\lambda=1$,

(a) $T\left(\widehat{\beta}_{F E}-\beta\right) \stackrel{p}{\rightarrow} \frac{-3 \varpi_{\varepsilon e}+6 \delta_{\varepsilon e}}{(1-\rho) \varpi_{\varepsilon}^{2}}$,

(b) $\sqrt{n} T\left(\widehat{\beta}_{F E}-\beta\right)-\sqrt{n} \tau_{3 n T}^{F E} \Rightarrow N\left(0, \kappa_{3}^{F E}\right)$,

where $\tau_{3 n T}^{F E}=\frac{\left(\frac{1}{n} \sum_{i=1}^{n} \frac{1}{T} \sum_{t=1}^{T}\left(x_{i t}-\bar{x}_{i}\right) \varepsilon_{i t}\right) \frac{\varpi_{\varepsilon e}}{\varpi_{\varepsilon}^{2}}+\delta_{\varepsilon e}}{(1-\rho) \frac{1}{n} \sum_{i=1}^{n} \frac{1}{T^{2}} \sum_{t=1}^{T}\left(x_{i t}-\bar{x}_{i}\right)^{2}}$ and $\kappa_{3}^{F E}=\frac{6 \varpi_{e \varepsilon}}{(1-\rho)^{2} \varpi_{\varepsilon}^{2}}$.

4. If $\rho=1$ and $\lambda=1$,

(a) $\widehat{\beta}_{F E}-\beta \stackrel{p}{\rightarrow} \frac{\varpi_{\varepsilon e}+6 \delta_{\varepsilon e}}{\varpi_{\varepsilon}^{2}}$,

(b) $\sqrt{n}\left(\widehat{\beta}_{F E}-\beta-\tau_{4 n T}^{F E}\right) \Rightarrow N\left(0, \kappa_{4}^{F E}\right)$,

where $\tau_{4 n T}^{F E}=\frac{\left(\frac{1}{n} \sum_{i=1}^{n} \frac{1}{T^{2}} \sum_{t=1}^{T}\left(x_{i t}-\bar{x}_{i}\right)^{2}\right) \frac{\varpi_{\varepsilon e}}{\varpi^{2}}+\delta_{\varepsilon e}}{\frac{1}{n} \sum_{i=1}^{n} \frac{1}{T^{2}} \sum_{t=1}^{T}\left(x_{i t}-\bar{x}_{i}\right)^{2}}$ and $\kappa_{4}^{F E}=\frac{2 \varpi_{e \cdot \varepsilon}}{5 \varpi_{\varepsilon}^{2}}$.

Note $\varpi_{\varepsilon e}$ is due to the endogeneity of the regressor $x_{i t}$, and $\delta_{\varepsilon e}$ is due to serial correlation. Because $u_{i t}-\bar{u}_{i}=\nu_{i t}-\bar{\nu}_{i}$, the individual effect $\mu_{i}$ is eliminated for each individual.

Corollary 2 When $E\left(e_{i t} \varepsilon_{i(t+k)}\right)=0$ for all $i$ and $k$, under the same conditions as for Theorem 2 , then

1. If $|\rho|<1$ and $|\lambda|<1$,

$\sqrt{n T}\left(\widehat{\beta}_{F E}-\beta\right) \Rightarrow N\left(0, \frac{\left(1-\lambda^{2}\right)^{2}\left[\psi_{00}+\sum_{r=1}^{\infty} \lambda^{2 r} \psi_{0 r}+\sum_{r=1}^{\infty} \rho^{2 r} \psi_{r 0}\right]}{(1-\rho \lambda)^{2} \sigma_{\varepsilon}^{4}}\right)$.

If $\varepsilon_{i t}$ and $e_{i t}$ are independent, $\sqrt{n T}\left(\widehat{\beta}_{F E}-\beta\right) \Rightarrow N\left(0, \frac{(1+\rho \lambda)\left(1-\lambda^{2}\right) \sigma_{e}^{2}}{(1-\rho \lambda)\left(1-\rho^{2}\right) \sigma_{\varepsilon}^{2}}\right)$. 
2. If $\rho=1$ and $|\lambda|<1$,

$$
\sqrt{n}\left(\widehat{\beta}_{F E}-\beta\right) \Rightarrow N\left(0, \frac{(1-\lambda)^{2} \sigma_{e}^{2}}{6 \sigma_{\varepsilon}^{2}}\right) .
$$

3. If $|\rho|<1$ and $\lambda=1$,

$$
\sqrt{n} T\left(\widehat{\beta}_{F E}-\beta\right) \Rightarrow N\left(0, \frac{6 \sigma_{e}^{2}}{(1-\rho)^{2} \sigma_{\varepsilon}^{2}}\right) \text {. }
$$

4. If $\rho=1$ and $\lambda=1$,

$$
\sqrt{n}\left(\widehat{\beta}_{F E}-\beta\right) \Rightarrow N\left(0, \frac{2 \sigma_{e}^{2}}{5 \sigma_{\varepsilon}^{2}}\right) \text {. }
$$

Corollary 2 follows directly from Theorem 2 . Note that case 1 is the textbook result under the assumptions of stationarity of the regressor and the disturbance term. Case 2 is new. Case 3 is discussed by Phillips and Moon (1999) and Kao and Chiang (2000). Case 4 is discussed in Kao (1999).

\section{$5 \quad$ FD Estimator}

The First-difference estimator of $\beta$ is given by

$$
\widehat{\beta}_{F D}=\frac{\sum_{i=1}^{n} \sum_{t=1}^{T}\left(x_{i t}-x_{i t-1}\right)\left(y_{i t}-y_{i t-1}\right)}{\sum_{i=1}^{n} \sum_{t=1}^{T}\left(x_{i t}-x_{i t-1}\right)^{2}} .
$$

Theorem 3 Under Assumptions 1 - 2, we obtain the following results:

1. If $|\rho|<1$ and $|\lambda|<1$,

(a) $\widehat{\beta}_{F D}-\beta \stackrel{p}{\rightarrow} \frac{\lim \frac{1}{n T} \sum_{i=1}^{n} \sum_{t=1}^{T} E\left[\left(x_{i t}-x_{i t-1}\right)\left(\nu_{i t}-\nu_{i t-1}\right)\right]}{\frac{2 \sigma_{\varepsilon}^{2}}{1+\lambda}+\frac{2\left(-2 \lambda^{2}+2 \lambda-1\right) \gamma_{\varepsilon}^{2}}{1-\lambda}}$,

(b) $\sqrt{n T}\left(\widehat{\beta}_{F D}-\beta-\tau_{1 n T}^{F D}\right) \Rightarrow N\left(0, \kappa_{1}^{F D}\right)$, where

$$
\tau_{1 n T}^{F D}=\frac{\lim \frac{1}{n T} \sum_{i=1}^{n} \sum_{t=1}^{T} E\left[\left(x_{i t}-x_{i t-1}\right)\left(\nu_{i t}-\nu_{i t-1}\right)\right]}{\frac{1}{n T} \sum_{i=1}^{n} \sum_{t=1}^{T}\left(x_{i t}-x_{i t-1}\right)^{2}},
$$

and

$$
\kappa_{1}^{F D}=\frac{(2-\lambda-\rho)^{2} \psi_{00}+\sum_{r=1}^{\infty}\left(-\rho^{r-1}+2 \rho^{r}-\rho^{r+1}\right)^{2} \psi_{0 r}+\sum_{r=1}^{\infty}\left(-\lambda^{r-1}+2 \lambda^{r}-\lambda^{r+1}\right)^{2} \psi_{r 0}}{(1-\rho \lambda)^{2}\left(\frac{2 \sigma_{\varepsilon}^{2}}{1+\lambda}+\frac{2\left(-2 \lambda^{2}+2 \lambda-1\right) \gamma_{\varepsilon}^{2}}{1-\lambda}\right)^{2}} .
$$

2. If $\rho=1$ and $|\lambda|<1$,

(a) $\widehat{\beta}_{F D}-\beta \stackrel{p}{\rightarrow} \frac{\lim \frac{1}{n T} \sum_{i=1}^{n} \sum_{t=1}^{T} E\left[\left(\varepsilon_{i t}+(\lambda-1) x_{i t-1}\right) e_{i t}\right]}{\frac{2 \sigma_{\varepsilon}^{2}}{1+\lambda}+\frac{2\left(-2 \lambda^{2}+2 \lambda-1\right) \gamma_{\varepsilon}^{2}}{1-\lambda}}$, 
(b) $\sqrt{n T}\left(\widehat{\beta}_{F E}-\beta-\tau_{2 n T}^{F D}\right) \Rightarrow N\left(0, \kappa_{2}^{F D}\right)$, where

$$
\tau_{2 n T}^{F D}=\frac{\lim \frac{1}{n T} \sum_{i=1}^{n} \sum_{t=1}^{T} E\left[\left(\varepsilon_{i t}+(\lambda-1) x_{i t-1}\right) e_{i t}\right]}{\frac{1}{n T} \sum_{i=1}^{n} \sum_{t=1}^{T}\left(x_{i t}-x_{i t-1}\right)^{2}},
$$

and

$$
\kappa_{2}^{F D}=\frac{2 \psi_{00}}{(1+\lambda)\left(\frac{2 \sigma_{\varepsilon}^{2}}{1+\lambda}+\frac{2\left(-2 \lambda^{2}+2 \lambda-1\right) \gamma_{\varepsilon}^{2}}{1-\lambda}\right)^{2}}
$$

3. If $|\rho|<1$ and $\lambda=1$,

(a) $\widehat{\beta}_{F D}-\beta \stackrel{p}{\rightarrow} \frac{1}{\sigma_{\varepsilon}^{2}} \lim \frac{1}{n T} \sum_{i=1}^{n} \sum_{t=1}^{T} E\left[\varepsilon_{i t}\left(\nu_{i t}-\nu_{i t-1}\right)\right]$,

(b) $\sqrt{n T}\left(\widehat{\beta}_{F D}-\beta-\tau_{3 n T}^{F D}\right) \Rightarrow N\left(0, \Pi_{3}^{F D}\right)$,

where $\tau_{3 n T}^{F D}=\frac{\lim \frac{1}{n T} \sum_{i=1}^{n} \sum_{t=1}^{T} E\left[\varepsilon_{i t}\left(\nu_{i t}-\nu_{i t-1}\right)\right]}{\frac{1}{n T} \sum_{i=1}^{n} \sum_{t=1}^{T}\left(x_{i t}-x_{i t-1}\right)^{2}}$ and $\kappa_{3}^{F D}=\frac{2 \psi_{00}}{(1+\rho) \sigma_{\varepsilon}^{4}}$.

4. If $\rho=1$ and $\lambda=1$,

(a) $\widehat{\beta}_{F D}-\beta \stackrel{p}{\rightarrow} \frac{\sigma_{\varepsilon e}}{\sigma_{\varepsilon}^{2}}$,

(b) $\sqrt{n T}\left(\widehat{\beta}_{F D}-\beta-\tau_{4 n T}^{F D}\right) \Rightarrow N\left(0, \kappa_{4}^{F D}\right)$, where $\tau_{4 n T}^{F D}=\frac{\sigma_{\varepsilon e}}{\frac{1}{n T} \sum_{i=1}^{n} \sum_{t=1}^{T}\left(x_{i t}-x_{i t-1}\right)^{2}}$ and $\kappa_{4}^{F D}=\frac{\varpi_{e . \varepsilon} \varpi_{\varepsilon}^{2}}{\sigma_{\varepsilon}^{4}}$.

Similar to the FE estimator, the individual effect $\mu_{i}$ is also eliminated by the FD estimator because $u_{i t}-u_{i t-1}=\nu_{i t}-\nu_{i t-1}$. In cases 2 and $4, \rho=1$, and the FD estimator is asymptotically equivalent to the GLS estimator because both methods transform the disturbance from $I(1)$ into $I(0)$. Actually, the FD estimator is mathematically the same as the GLS estimator except for the omission of the first observation for each individual.

Corollary 3 When $E\left(e_{i t} \varepsilon_{i(t+k)}\right)=0$ for all $i$ and $k$, under the same conditions as for Theorem 3, then

1. If $|\rho|<1$ and $|\lambda|<1$,

$\sqrt{n T}\left(\widehat{\beta}_{F D}-\beta\right) \Rightarrow N\left(0, \frac{(1+\lambda)^{2}\left[(2-\lambda-\rho)^{2} \psi_{00}+\sum_{r=1}^{\infty}\left(-\rho^{r-1}+2 \rho^{r}-\rho^{r+1}\right)^{2} \psi_{0 r}+\sum_{r=1}^{\infty}\left(-\lambda^{r-1}+2 \lambda^{r}-\lambda^{r+1}\right)^{2} \psi_{r 0}\right]}{4(1-\rho \lambda)^{2} \sigma_{\varepsilon}^{4}}\right)$.

If $\varepsilon_{i t}$ and $e_{i t}$ are independent, $\sqrt{n T}\left(\widehat{\beta}_{F D}-\beta\right) \Rightarrow N\left(0, \frac{(1+\lambda)^{2}\left[(2-\rho-\lambda)^{2}+\frac{(1-\rho)^{3}}{1+\rho}+\frac{(1-\lambda)^{3}}{1+\lambda}\right] \sigma_{e}^{2}}{4(1-\rho \lambda)^{2} \sigma_{\varepsilon}^{2}}\right)$.

2. If $\rho=1$ and $|\lambda|<1$,

$\sqrt{n T}\left(\widehat{\beta}_{F D}-\beta\right) \Rightarrow N\left(0, \frac{(1+\lambda) \psi_{00}}{2 \sigma_{\varepsilon}^{4}}\right)$.

If $\varepsilon_{i t}$ and $e_{i t}$ are independent, $\sqrt{n T}\left(\widehat{\beta}_{F E}-\beta\right) \Rightarrow N\left(0, \frac{(1+\lambda) \sigma_{e}^{2}}{2 \sigma_{\varepsilon}^{2}}\right)$. 
3. If $|\rho|<1$ and $\lambda=1$,

$$
\begin{aligned}
& \sqrt{n T}\left(\widehat{\beta}_{F D}-\beta\right) \Rightarrow N\left(0, \frac{2 \psi_{00}}{(1+\rho) \sigma_{\varepsilon}^{4}}\right) . \\
& \text { If } \varepsilon_{i t} \text { and } e_{i t} \text { are independent, } \sqrt{n T}\left(\widehat{\beta}_{F D}-\beta\right) \Rightarrow N\left(0, \frac{2 \sigma_{e}^{2}}{(1+\rho) \sigma_{\varepsilon}^{2}}\right) .
\end{aligned}
$$

4. If $\rho=1$ and $\lambda=1$,

$$
\sqrt{n T}\left(\widehat{\beta}_{F D}-\beta\right) \Rightarrow N\left(0, \frac{\sigma_{e}^{2}}{\sigma_{\varepsilon}^{2}}\right) \text {. }
$$

Corollary 3 follows directly from Theorem 3 .

\section{GLS Estimator}

Let us rewrite equation (1) in vector form

$$
\mathbf{y}=\alpha \boldsymbol{\iota}_{n T}+\mathbf{x} \beta+\mathbf{u}
$$

where $\mathbf{y}$ is $n T \times 1, \mathbf{x}$ is a vector of $x_{i t}$ of dimension $n T \times 1, \boldsymbol{\iota}_{n T}$ is a vector of ones of dimension $n T$. and $\mathbf{u}$ is $n T \times 1$. As shown in the Appendix,

$$
\widehat{\beta}_{G L S}=\left[\mathbf{x}^{\prime} \Phi^{-1} \mathbf{x}-\mathbf{x}^{\prime} \Phi^{-1} \iota_{n T}\left(\iota_{n T}^{\prime} \Phi^{-1} \iota_{n T}\right)^{-1} \iota_{n T}^{\prime} \Phi^{-1} \mathbf{x}\right]^{-1}\left[\mathbf{x}^{\prime} \Phi^{-1} \mathbf{y}-\mathbf{x}^{\prime} \Phi^{-1} \iota_{n T}\left(\boldsymbol{\iota}_{n T}^{\prime} \Phi^{-1} \iota_{n T}\right)^{-1} \iota_{n T}^{\prime} \Phi^{-1} \mathbf{y}\right]
$$

and

$\widehat{\beta}_{G L S}-\beta=\left[\mathbf{x}^{\prime} \Phi^{-1} \mathbf{x}-\mathbf{x}^{\prime} \Phi^{-1} \iota_{n T}\left(\boldsymbol{\iota}_{n T}^{\prime} \Phi^{-1} \iota_{n T}\right)^{-1} \boldsymbol{\iota}_{n T}^{\prime} \Phi^{-1} \mathbf{x}\right]^{-1}\left[\mathbf{x}^{\prime} \Phi^{-1} \mathbf{u}-\mathbf{x}^{\prime} \Phi^{-1} \iota_{n T}\left(\boldsymbol{\iota}_{n T}^{\prime} \Phi^{-1} \iota_{n T}\right)^{-1} \boldsymbol{\iota}_{n T}^{\prime} \Phi^{-1} \mathbf{u}\right]$,

where $\Phi=E\left(\mathbf{u u}^{\prime}\right)$.

One can decompose the variance-covariance matrix into

$$
\Phi=E\left(\mathbf{u u}^{\prime}\right)=\sigma_{\mu}^{2}\left(I_{n} \otimes \iota_{T} \boldsymbol{\iota}_{T}^{\prime}\right)+\varpi_{e}^{2}\left(I_{n} \otimes \mathbf{A}\right)
$$

where $\iota_{T}$ is a vector of ones of dimension T. A is the variance-covariance matrix of $\nu_{i t}$,

$$
\mathbf{A}=\left[\begin{array}{ccccc}
1 & \rho & \rho^{2} & \cdots & \rho^{T-1} \\
\rho & 1 & \rho & \cdots & \rho^{T-2} \\
\rho^{2} & \rho & 1 & \cdots & \rho^{T-3} \\
\vdots & \vdots & \vdots & \ddots & \vdots \\
\rho^{T-1} & \rho^{T-2} & \rho^{T-3} & \cdots & 1
\end{array}\right]
$$


when $|\rho|<1$ and

$$
\mathbf{A}=\left[\begin{array}{ccccc}
1 & 1 & 1 & \cdots & 1 \\
1 & 2 & 2 & \cdots & 2 \\
1 & 2 & 3 & \cdots & 3 \\
\vdots & \vdots & \vdots & \ddots & \vdots \\
1 & 2 & 3 & \cdots & T
\end{array}\right]
$$

when $\rho=1$. Thus, it can be shown that

$$
\Phi^{-1}=I_{n} \otimes\left[\frac{1}{\varpi_{e}^{2}}\left(\mathbf{A}^{-1}-\frac{\sigma_{\mu}^{2}}{\varpi_{e}^{2}+\theta \sigma_{\mu}^{2}} \mathbf{A}^{-1} \iota_{T} \iota_{T}^{\prime} \mathbf{A}^{-1}\right)\right]
$$

where $\theta=\boldsymbol{\iota}_{T}^{\prime} \mathbf{A}^{-1} \iota_{T}$.

When $|\rho|<1$, this estimation is equivalent to the Prais-Winsten transformation method suggested by Baltagi and Li (1991). One can easily verify that $\mathbf{A}^{-1}=\mathbf{C C}^{\prime}$, where

$$
\mathbf{C}=\left[\begin{array}{cccccc}
\sqrt{1-\rho^{2}} & 0 & 0 & \cdots & 0 & 0 \\
-\rho & 1 & 0 & \cdots & 0 & 0 \\
0 & -\rho & 1 & \cdots & 0 & 0 \\
\vdots & \vdots & \vdots & \ddots & \vdots & \vdots \\
0 & 0 & 0 & -\rho & 1 & 0 \\
0 & 0 & 0 & 0 & -\rho & 1
\end{array}\right]
$$

is the Prais-Winsten transformation matrix as in Baltagi and Li (1991).

Thus, we have the following theorem:

Theorem 4 Under Assumptions 1 - 2, we obtain the following results:

1. If $|\rho|<1$ and $|\lambda|<1$,
(a) $\left(\widehat{\beta}_{G L S}-\beta\right) \stackrel{p}{\rightarrow} \frac{\lim \frac{1}{T} \sum_{t=1}^{T} E\left[\left(x_{i t}-\rho x_{i t-1}\right) e_{i t}\right]}{\frac{\left(1-2 \rho \lambda+\rho^{2}\right) \sigma_{\varepsilon}^{2}}{1-\lambda^{2}}+\frac{2\left(\lambda-2 \rho \lambda^{2}+\rho^{2} \lambda-\rho\right) \gamma_{\varepsilon}^{2}}{1-\lambda}}$
(b) $\sqrt{n T}\left(\widehat{\beta}_{G L S}-\beta-\tau_{1 n T}^{G L S}\right) \Rightarrow N\left(0, \kappa_{1}^{G L S}\right)$, where $\tau_{1 n T}^{G L S}=\frac{\lim \frac{1}{T} \sum_{t=1}^{T} E\left[\left(x_{i t}-\rho x_{i t-1}\right) e_{i t}\right]}{\sigma_{e}^{2} \frac{1}{n T} X^{\prime} \Phi^{-1} X}, \kappa_{1}^{G L S}=\frac{\left(1-2 \rho \lambda+\rho^{2}\right) \psi_{00}}{\left(1-\lambda^{2}\right)\left[\frac{\left(1-2 \rho \lambda+\rho^{2}\right) \sigma_{\varepsilon}^{2}}{1-\lambda^{2}}+\frac{2\left(\lambda-2 \rho \lambda^{2}+\rho^{2} \lambda-\rho\right) \gamma_{\varepsilon}^{2}}{1-\lambda}\right]^{2}}$

2. If $\rho=1$ and $|\lambda|<1$, 
(a) $\left(\widehat{\beta}_{G L S}-\beta\right) \stackrel{p}{\rightarrow} \frac{\lim \frac{1}{n} \frac{1}{T} \sum_{i=1}^{n} \sum_{t=1}^{T} E\left[\left(x_{i t}-x_{i t-1}\right) e_{i t}\right]}{\frac{2 \sigma_{\varepsilon}^{2}}{1+\lambda}+\frac{2\left(-2 \lambda^{2}+2 \lambda-1\right) \gamma_{\varepsilon}^{2}}{1-\lambda}}$,

(b) $\sqrt{n T}\left(\widehat{\beta}_{G L S}-\beta-\tau_{2 n T}^{G L S}\right) \Rightarrow N\left(0, \kappa_{2}^{G L S}\right)$, where $\tau_{2 n T}^{G L S}=\frac{\lim \frac{1}{n} \sum_{i=1}^{n} \frac{1}{T} \sum_{t=1}^{T} E\left[\left(x_{i t}-x_{i t-1}\right) e_{i t}\right]}{\sigma_{e}^{2} \frac{1}{n T} X^{\prime} \Phi^{-1} X}, \kappa_{2}^{G L S}=\frac{2 \psi_{00}}{(1+\lambda)\left[\frac{2 \sigma_{\varepsilon}^{2}}{1+\lambda}+\frac{2\left(-2 \lambda^{2}+2 \lambda-1\right) \gamma_{\varepsilon}^{2}}{1-\lambda}\right]^{2}}$

3. If $|\rho|<1$ and $\lambda=1$,

(a) $T\left(\widehat{\beta}_{G L S}-\beta\right) \stackrel{p}{\rightarrow} \frac{-3 \varpi_{\varepsilon e}+6 \gamma_{\varepsilon e}+\frac{6}{1-\rho} \sigma_{\varepsilon e}}{(1-\rho) \varpi_{\varepsilon}^{2}}$,

(b) $\sqrt{n} T\left(\widehat{\beta}_{G L S}-\beta\right)-\sqrt{n} \tau_{3 n T}^{G L S} \Rightarrow N\left(0, \kappa_{3}^{G L S}\right)$,

where $\tau_{3 n T}^{G L S}=\frac{\frac{1}{n} \sum_{i=1}^{n}(1-\rho)\left(\left(\frac{1}{n} \sum_{i=1}^{n} \frac{1}{T} \sum_{t=1}^{T}\left(x_{i t}-\bar{x}_{i}\right) \varepsilon_{i t}\right) \frac{\varpi_{\varepsilon e}}{\varpi_{\varepsilon}^{2}}+\gamma_{\varepsilon e}+\frac{\sigma_{\varepsilon e}}{1-\rho}\right)}{\sigma_{e}^{2} \frac{1}{n T} X^{\prime} \Phi^{-1} X}, \kappa_{3}^{G L S}=\frac{6 \varpi_{e \varepsilon}}{(1-\rho)^{2} \varpi_{\varepsilon}^{2}}$.

4. If $\rho=1$ and $\lambda=1$,

(a) $\sqrt{T}\left(\widehat{\beta}_{G L S}-\beta\right) \stackrel{p}{\rightarrow} \frac{\sigma_{\varepsilon e}}{\sigma_{\varepsilon}^{2}}$,

(b) $\sqrt{n T}\left(\widehat{\beta}_{G L S}-\beta\right)-\sqrt{n} \tau_{4 n T}^{G L S} \Rightarrow N\left(0, \kappa_{4}^{G L S}\right)$, where $\tau_{4 n T}^{G L S}=\frac{\sqrt{n} \sigma_{\varepsilon e}}{\sigma_{e}^{2} \frac{1}{n T} X^{\prime} \Phi^{-1} X}, \kappa_{4}^{G L S}=\frac{\varpi_{e}^{2} \varpi_{\varepsilon}^{2}}{\sigma_{\varepsilon}^{4}}$.

It is well known that the random effects model imposes the critical assumption that $\mu_{i}$ needs to be independent of $x_{i t}$. It is worth pointing out that this assumption is only needed for the case $\rho<1$. When $\rho=1$, the GLS transformation is identical to the first-difference estimation except for the first observation of each individual. The Cochrane-Orcutt (CO) procedure of GLS transformation ignores the information contained in the first observation. Hence, if one use Cochrane-Orcutt (CO) procedure, the GLS estimation will be the same as the FD estimation and $\mu_{i}$ need not to be independent of $x_{i t}$ when $\rho=1$. When $\rho<1$, $E\left(\mu_{i} \mid x_{i t}\right)=0$ is required, otherwise $\hat{\beta}_{G L S}$ would be biased and inconsistent. In this case, one may use the within or first-difference transformation to wipe out $\mu_{i}$ and then run GLS estimation. The Within-GLS or FD-GLS estimators can be shown asymptotically as efficient as GLS estimator. However, this is beyond the scope of this paper and can be left as a further extension. The following corollary follows directly from Theorem 4.

Corollary 4 When $E\left(e_{i t} \varepsilon_{i(t+k)}\right)=0$ for all $i$ and $k$, under the same conditions as for Theorem 4 , then

1. If $|\rho|<1$ and $|\lambda|<1$, $\sqrt{n T}\left(\widehat{\beta}_{G L S}-\beta\right) \Rightarrow N\left(0, \frac{\left(1-\lambda^{2}\right) \psi_{00}}{\left(1-2 \rho \lambda+\rho^{2}\right) \sigma_{\varepsilon}^{4}}\right)$.

If $\varepsilon_{i t}$ and $e_{i t}$ are independent, $\sqrt{n T}\left(\widehat{\beta}_{G L S}-\beta\right) \Rightarrow N\left(0, \frac{\left(1-\lambda^{2}\right) \sigma_{e}^{2}}{\left(1-2 \rho \lambda+\rho^{2}\right) \sigma_{\varepsilon}^{2}}\right)$. 
2. If $\rho=1$ and $|\lambda|<1$,

$$
\begin{aligned}
& \sqrt{n T}\left(\widehat{\beta}_{G L S}-\beta\right) \Rightarrow N\left(0, \frac{(1+\lambda) \psi_{00}}{2 \sigma_{\varepsilon}^{4}}\right) . \\
& \text { If } \varepsilon_{i t} \text { and } e_{i t} \text { are independent, } \sqrt{n T}\left(\widehat{\beta}_{G L S}-\beta\right) \Rightarrow N\left(0, \frac{(1+\lambda) \sigma_{e}^{2}}{2 \sigma_{\varepsilon}^{2}}\right) .
\end{aligned}
$$

3. If $|\rho|<1$ and $\lambda=1$,

$$
\sqrt{n} T\left(\widehat{\beta}_{G L S}-\beta\right) \Rightarrow N\left(0, \frac{6 \sigma_{e}^{2}}{(1-\rho)^{2} \sigma_{\varepsilon}^{2}}\right) \text {. }
$$

4. If $\rho=1$ and $\lambda=1$,

$$
\sqrt{n T}\left(\widehat{\beta}_{G L S}-\beta\right) \Rightarrow N\left(0, \frac{\sigma_{e}^{2}}{\sigma_{\varepsilon}^{2}}\right) \text {. }
$$

Case 1 is the textbook result. Case 3 is discussed in Choi (1999). Cases 2 and 4 are new.

\section{Feasible GLS Estimator}

It is clear that the GLS estimator in Section 6 is not feasible. In this section, we discuss feasible GLS estimation. Assuming $E\left(e_{i t} \varepsilon_{i(t+k)}\right)=0$ for all $i$ and $k$ and $\varepsilon_{i t}$ and $e_{i t}$ are independent, a feasible GLS estimator can be calculated by estimating the autocorrelation coefficient $\rho$ and the variance components $\sigma_{\mu}^{2}$ and $\sigma_{e}^{2}$. To estimate these parameters, we take the following steps.

First, retrieve the residual estimator $\widehat{\nu}_{i t}$ from the FE regression in (1). Now $\rho$ can be estimated as the correlation between $\widehat{\nu}_{i t}$ and $\widehat{\nu}_{i t-1}$, i.e.,

$$
\widehat{\rho}=\frac{\sum_{i=1}^{n} \sum_{t=2}^{T}\left(\widehat{\nu}_{i t}-\overline{\hat{\nu}}\right)\left(\widehat{\nu}_{i t-1}-\overline{\hat{\nu}}\right)}{\sqrt{\sum_{i=1}^{n} \sum_{t=2}^{T}\left(\widehat{\nu}_{i t-1}-\overline{\hat{\nu}}\right)^{2}} \sqrt{\sum_{i=1}^{n} \sum_{t=2}^{T}\left(\widehat{\nu}_{i t-1}-\overline{\hat{\nu}}\right)^{2}}},
$$

where $\overline{\hat{\nu}}$ is the sample average of $\widehat{\nu}_{i t}$. Alternatively, as suggested by Baltagi and Li (1991), one can estimate $\rho$ by

$$
\widehat{\rho}=\sum_{i=1}^{n} \sum_{t=2}^{T} \widehat{\nu}_{i t} \widehat{\nu}_{i t-1} / \sum_{i=1}^{n} \sum_{t=2}^{T}\left(\widehat{\nu}_{i t-1}\right)^{2} .
$$

Baltagi and Li (1997) suggests another consistent estimator $\widehat{\rho}=\left(\tilde{Q}_{1}-\tilde{Q}_{2}\right) /\left(\tilde{Q}_{0}-\tilde{Q}_{1}\right)$, where $\tilde{Q}_{s}=$ $\sum_{i=1}^{n} \sum_{t=s+1}^{T} \widehat{u}_{i t} \widehat{u}_{i t-s} / n(T-s)$. We choose the correlation coefficient estimator because it ensures that $\widehat{\rho}$ is always between 0 and 1 . It can be shown that $\widehat{\rho}$ in (14) is a consistent estimator of $\rho$ by using the Theorem 2, i.e., $\widehat{\rho} \stackrel{p}{\rightarrow} \rho$ if $|\rho|<1$.

Next, using the FE residuals $\widehat{\nu}_{i t}$ and the estimate of the autocorrelation coefficient $\widehat{\rho}$, we can get $\widehat{e}_{i t}$. Therefore $\sigma_{e}^{2}$ can be estimated by $\hat{\sigma}_{e}^{2}=\frac{1}{n T} \sum_{i=1}^{N} \sum_{t=1}^{T} \widehat{e}_{i t}^{2}$. Also, $\sigma_{\mu}^{2}$ can be estimated using $\hat{\sigma}_{\mu}^{2}=$ 
$\frac{1}{n T} \sum_{i=1}^{N} \sum_{t=1}^{T}\left(\widehat{u}_{i t}^{2}-\widehat{\nu}_{i t}^{2}\right)$, where $\widehat{u}_{i t}$ denote the OLS residuals from equation (10). $\hat{\sigma}_{e}^{2}$ and $\hat{\sigma}_{\mu}^{2}$ are consistent estimators for $\sigma_{e}^{2}$ and $\sigma_{\mu}^{2}$ respectively, i.e., $\hat{\sigma}_{e}^{2} \stackrel{p}{\rightarrow} \sigma_{e}^{2}, \hat{\sigma}_{\mu}^{2} \stackrel{p}{\rightarrow} \sigma_{\mu}^{2}$ if $|\rho|<1$. These variance components can be estimated by using the variance decomposition and the Prais-Winsten (PW) transformation suggested by Baltagi and Li (1991). Alternatively, one can also use the Cochrane-Orcutt (CO) procedure, which ignores the information contained in the first observation. As suggested by Maeshiro (1976), Beach and MacKinnon (1978) and Park and Mitchell (1980), estimation using the PW transformation is more efficient than using the $\mathrm{CO}$ procedure when the regressors are trended.

When the assumptions of corollary 4 hold, one can show that feasible GLS has the same asymptotic distribution as true GLS. Define $\phi=\left(\rho, \sigma_{\mu}^{2}, \sigma_{e}^{2}\right)$ and $\hat{\phi}$ is its corresponding estimator. Then $\Phi=\Phi(\phi)$. Further define that $G_{k}(\phi)=\partial \Phi^{-1}(\phi) / \partial \phi_{k}$, where $k=1,2,3$. For example, in case 1 , a Taylor's series expansion as in Fuller and Battese (1973) gives

$$
\begin{aligned}
& \sqrt{n T}\left(\hat{\tau}_{G L S}-\tau\right) \\
= & \left(\frac{Z^{\prime} \Phi^{-1}(\hat{\phi}) Z}{n T}\right)^{-1}\left(\frac{Z^{\prime} \Phi^{-1}(\hat{\phi}) u}{\sqrt{n T}}\right) \\
= & \left(\frac{Z^{\prime} \Phi^{-1}(\phi) Z}{n T}\right)^{-1}\left(\frac{Z^{\prime} \Phi^{-1}(\phi) u}{\sqrt{n T}}\right)+\sum_{k=1}^{3}\left[\left(\frac{Z^{\prime} \Phi^{-1}\left(\phi^{*}\right) Z}{n T}\right)^{-1}\left(\frac{Z^{\prime} G_{k}\left(\phi^{*}\right) u}{\sqrt{n T}}\right)\right. \\
& \left.-\left(\frac{Z^{\prime} \Phi^{-1}\left(\phi^{*}\right) Z}{n T}\right)^{-1}\left(\frac{Z^{\prime} G_{k}\left(\phi^{*}\right) Z}{n T}\right)\left(\frac{Z^{\prime} \Phi^{-1}\left(\phi^{*}\right) Z}{n T}\right)^{-1}\left(\frac{Z^{\prime} \Phi^{-1}\left(\phi^{*}\right) u}{\sqrt{n T}}\right)\right](\hat{\phi}-\phi) \\
= & \left(\frac{Z^{\prime} \Phi^{-1}(\phi) Z}{n T}\right)^{-1}\left(\frac{Z^{\prime} \Phi^{-1}(\phi) u}{\sqrt{n T}}\right)+o_{p}(1)
\end{aligned}
$$

where $\phi^{*}$ lies between $\hat{\phi}$ and $\phi$, hence $\phi^{*} \stackrel{p}{\rightarrow} \phi$. The last equal sign holds if $\frac{Z^{\prime} \Phi^{-1}(\phi) Z}{n T}=O_{p}(1), \frac{Z^{\prime} G_{k}\left(\phi^{*}\right) Z}{n T}=$ $O_{p}(1), \frac{Z^{\prime} \Phi^{-1}\left(\phi^{*}\right) u}{\sqrt{n T}}=O_{p}(1)$ and $\frac{Z^{\prime} G_{k}\left(\phi^{*}\right) u}{\sqrt{n T}}=O_{p}(1)$. This follows using similar arguments in the proofs of the Theorems above. The proofs are similar for the remaining three cases and are omitted to save space.

\section{Efficiency Comparisons}

This section summarizes the relative efficiency of OLS, FE, GLS and FD estimators. First, the speed of convergence for the different cases considered are summarized as follows: 


\section{OLS FE FD GLS}

Case $1:|\rho|<1$ and $|\lambda|<1 \quad \sqrt{n T} \quad \sqrt{n T} \quad \sqrt{n T} \quad \sqrt{n T}$

Case 2: $\rho=1$ and $|\lambda|<1 \quad \sqrt{n} \quad \sqrt{n} \quad \sqrt{n T} \quad \sqrt{n T}$

Case 3: $|\rho|<1$ and $\lambda=1 \quad \sqrt{n T} \quad \sqrt{n} T \quad \sqrt{n T} \quad \sqrt{n} T$

Case 4: $\rho=1$ and $\lambda=1 \quad \sqrt{n} \quad \sqrt{n} \quad \sqrt{n T} \quad \sqrt{n T}$

In case 1 , the four estimators have the same convergence speed of $\sqrt{n T}$. The efficiency of the OLS estimator is hard to compare with the remaining estimators because OLS does not difference out $\mu_{i}$, and as a result its variance still contains $\sigma_{\mu}^{2}$. That GLS is more efficient than FE and FD is evident from the Gauss-Markov theorem. Since these estimators all converge at same rate $\sqrt{n T}$, we plot the relative efficiency of the FE and FD estimators with respect to true GLS in Figure 1 and 2. The relative efficiency of the FE estimator with respect to true GLS is given by

$$
\begin{aligned}
\operatorname{var}\left(\widehat{\beta}_{F E}\right) / \operatorname{var}\left(\widehat{\beta}_{G L S}\right) & =\frac{(1+\rho \lambda)\left(1-\lambda^{2}\right) \sigma_{e}^{2}}{(1-\rho \lambda)\left(1-\rho^{2}\right) \sigma_{\varepsilon}^{2}} / \frac{\left(1-\lambda^{2}\right) \sigma_{e}^{2}}{\left(1-2 \rho \lambda+\rho^{2}\right) \sigma_{\varepsilon}^{2}} \\
& =\frac{(1+\rho \lambda)\left(1-2 \rho \lambda+\rho^{2}\right)}{(1-\rho \lambda)\left(1-\rho^{2}\right)}
\end{aligned}
$$

The relative efficiency of the FD estimator with respect to true GLS is given by

$$
\begin{aligned}
\operatorname{var}\left(\widehat{\beta}_{F D}\right) / \operatorname{var}\left(\widehat{\beta}_{G L S}\right) & =\frac{(1+\lambda)^{2}\left[(2-\rho-\lambda)^{2}+\frac{(1-\rho)^{3}}{1+\rho}+\frac{(1-\lambda)^{3}}{1+\lambda}\right] \sigma_{e}^{2}}{4(1-\rho \lambda)^{2} \sigma_{\varepsilon}^{2}} / \frac{\left(1-\lambda^{2}\right) \sigma_{e}^{2}}{\left(1-2 \rho \lambda+\rho^{2}\right) \sigma_{\varepsilon}^{2}} \\
& =\frac{(1+\lambda)\left(1-2 \rho \lambda+\rho^{2}\right)\left[(2-\rho-\lambda)^{2}+\frac{(1-\rho)^{3}}{1+\rho}+\frac{(1-\lambda)^{3}}{1+\lambda}\right]}{4(1-\lambda)(1-\rho \lambda)^{2}} .
\end{aligned}
$$

One can easily verify that both relative efficiencies are larger or equal to 1. Comparing the GLS estimator with the FE and FD estimators, the relative efficiency depends on the values of $\rho$ and $\lambda$. As shown in Figure 1 and 2 , when $\rho$ is small, the FE estimator performs well in terms of relative efficiency with respect to true GLS. When $\rho$ is large, the FD estimators performs well in terms of relative efficiency with respect to true GLS.

In case 2 , the disturbance is $I(1)$ but the regressor is $I(0)$. The noise is strong so that it dominates the signal. In the time series case, the OLS estimator is not consistent. After double smoothing using panel data, the asymptotic distribution becomes normal and the convergence speed is $\sqrt{n}$. GLS estimation, however, transforms the disturbance into $I(0)$. Therefore the convergence speed is $\sqrt{n T}$. When the disturbance is $I(1)$, first-difference estimation will be the same as GLS except for the first observation. Hence it is also $\sqrt{n T}$ consistent. 
In case 3 , the disturbance is $I(0)$ but the regressor is $I(1)$. This is the cointegration case. The cointegration literature shows that the GLS estimators is $T$ consistent in time series models. In the panel data model, both GLS and FE are $\sqrt{n} T$ consistent.

In case 4 , both the disturbance and the regressor are $I(1)$. This is the spurious regression case. As shown in Kao (1999), the FE estimator is $\sqrt{n}$ consistent. For the same reason given in case 2, first-differencing transforms the disturbance term from $I(1)$ to $I(0)$. Therefore, the convergence speed of both the GLS or the FD estimators is $\sqrt{n T}$.

In case 3 , the $\mathrm{FE}$ estimator is more efficient than the FD estimator when $v_{i t}$ are stationary, including the special case when $v_{i t}$ are serially uncorrelated. In cases 2 and 4 , the FD estimator is more efficient when $v_{i t}$ follows a random walk. These results verify the conclusion in Wooldridge (2002). However, in case 1, when $\rho$ is large, even though $v_{i t}$ does not follow a random walk, the FD estimator is still more efficient than the FE estimator.

\section{Monte Carlo Simulation}

This section reports the results of Monte Carlo experiments designed to investigate the finite sample relative efficiency of the OLS, FE, FD, GLS-CO, GLS-PW estimators with respect to true GLS. The model is generated by

$$
y_{i t}=x_{i t} \beta+\mu_{i}+v_{i t}, \quad i=1, \ldots, n, \quad t=1, \ldots, T
$$

with $\beta=10, \mu_{i} \stackrel{i i d}{\sim} N(0,5)$ and $v_{i t}$ and $x_{i t}$ follow an $\mathrm{AR}(1)$ process given in (2) and (3), respectively with $\rho$ and $\lambda$ varying over the range $(0,0.2,0.4,0.6,0.8,0.9,1)$ and $\sigma_{\varepsilon}^{2}=\sigma_{e}^{2}=5$. The sample sizes $n$ and $T$ are varied over the range $(20,40,60,120,240)$. For each experiment, we perform 10, 000 replications. For each replication we estimate the model using OLS, FE, FD, GLS-CO, GLS-PW and true GLS. Even with this modest design we had 1225 experiments. GAUSS for Windows 6.0 was used to perform the simulations. Random numbers for $\mu_{i}$ and $\varepsilon_{i t}$ were generated by the GAUSS procedure RNDNS. We generated $n(T+1000)$ random numbers and then split them into $n$ series so that each series had the same mean and variance. The first 1,000 observations were discarded for each series.

Tables 1-3 give the relative mean square error (MSE) of each estimator of $\beta$ with respect to true GLS for various values of $\rho, \lambda, n$, and $T$. We only report 3 Tables to give a flavour of the results, the rest are available upon request from the authors. Several conclusions emerge from these results. First, the true GLS estimator is the most efficient one in terms of mean squared error. Its efficiency gain over the OLS estimator is enormous particularly when $\rho$ and/or $\lambda$ is large. Second, the FE estimator is less efficient than true GLS, 
but more efficient than the feasible GLS estimator when $\rho=0$. However, when $\rho$ increases, the feasible GLS estimator quickly becomes more efficient than the FE estimator. Third, the FD estimator is also less efficient than true GLS. When $\rho$ increases, the FD estimator becomes as efficient as the GLS estimator. Interestingly, the FD estimator behaves poorly when $\lambda$ is close to 1 but $\rho$ is small. Fourth, the feasible GLS estimator is slightly less efficient than the true GLS estimator and beats OLS, FE and FD as long as $\rho>0.2$. In summary, our simulation results show that the feasible GLS estimator performs well, and is second best only to true GLS when $\rho>0.2$

\section{Conclusion}

In this paper, we compared the efficiency of OLS, FE, FD, and GLS estimators in panel models with $I(0)$ and $I(1)$ regressor and regression error. When the regression error is $I(0)$ and the regressor is $I(1)$ and hence the model is cointegrated, both the FE and GLS estimators are asymptotically efficient. When the regression error is $I(1)$ and the regressor is $I(1)$ and hence the model is spurious, the FE and GLS estimators are $\sqrt{n}$ and $\sqrt{n T}$ consistent, respectively. This implies that GLS is the preferred estimator as far as the regression error specification is concerned since GLS converges at as good or better rate in both cases (i.e., regression error is either $I(0)$ or $I(1))$. 


\section{References}

[1] Baltagi, B. (2005), Econometric Analysis of Panel Data, New York, Wiley.

[2] Baltagi, B., and Kao, C. (2000), "Nonstationary Panels, Cointegration in Panels and Dynamic Panels: A Survey", Advances in Econometrics, 15, 7-51.

[3] Baltagi, B. H., and Krämer, W. (1997), 97.2.1. A Simple Linear Trend Model with Error Components, Econometric Theory, 13, 463-463.

[4] Baltagi, B. H., and Li, Q. (1991), "A Transformation That Will Circumvent the Problem of Autocorrelation in an Error Component Model," Journal of Econometrics, 52, 371-380.

[5] Baltagi, B. H., and Li, Q. (1997), "Monte Carlo Results on Pure and Pretest Estimators of an Error Component Model with Autocorrelated Disturbances," Annales D'Économie et de Statistique, 48, 69-82.

[6] Beach, B. M., and MacKinnon, J. G. (1978), "A Maximum Likelihood Procedure for Regression with Autocorrelation Errors," Econometrica, 46, 51-58.

[7] Breitung, J., and Pesaran, M. H. (2005), "Unit Roots and Cointegration in Panels," forthcoming in L. Matyas, and P. Sevestre, The Econometrics of Panel Data (Third Edition), Kluwer Academic Publishers..

[8] Choi, I. (1999), "Asymptotic Analysis of a Nonstationary Error Component Model," Working paper, Kookmin University, Korea.

[9] Choi, I. (2002), "Instrumental Variables Estimation of a Nearly Nonstationary, Heterogeneous Error Component Model," Journal of Econometrics, 109, 1-32.

[10] Choi, I. (2006), "Nonstationary Panels," Palgrave Handbooks of Econometrics, Vol. 1, 511-539. Palgrave Macmillan: New York.

[11] Choi, C., Hu, L., and Ogaki, M. (2004), "A Spurious Regression Approach to Estimating Structural Parameters"," Working paper, Ohio State University, US.

[12] Fuller, W., and Battese, G. E. (1973), "Transformations for Estimation of Linear Models with NestedError Structure," Journal of the American Statistical Association, 68, 626-632.

[13] Hsiao, C. (1986), Analysis of Panel Data, Cambridge, Cambridge University Press. 
[14] Kao, C. (1999), "Spurious Regression and Residual-Based Tests for Cointegration in Panel Data," Journal of Econometrics, 90, 1-44.

[15] Kao, C., and Chiang, M-H. (2000), "On the Estimation and Inference of a Cointegrated Regression in Panel Data," Advances in Econometrics, 15, 179-222.

[16] Kao, C., and Emerson, J. (2002a), "Testing for Structural Change of a Time Trend Regression in Panel Data: Part I," Journal of Propagations in Probability and Statistics, 2, 57-75.

[17] Kao, C., and Emerson, J. (2002b), "Testing for Structural Change of a Time Trend Regression in Panel Data: Part II," Journal of Propagations in Probability and Statistics, 2, 207-250.

[18] Maeshiro, A. (1976), "Autoregressive Transformation, Trended Independent Variables and Autocorrelated Disturbance Terms," The Review of Economics and Statistics, 58, 497-500.

[19] Park, S. J., and Mitchell, B. M. (1980), "Estimating the Autocorrelated Error Model with Trended Data," Journal of Econometrics, 13, 185-201.

[20] Phillips, P. C. B., (1986), "Understanding spurious regressions in econometrics," Journal of Econometrics, 33, 311-340.

[21] Phillips, P. C. B., and Moon, H., (1999), "Linear Regression Limit Theory for Nonstationary Panel Data," Econometrica, 67, 1057-1111.

[22] Phillips, P. C. B., and Park, J. Y. (1988), "Asymptotic Equivalence of Ordinary Least Squares and Generalized Least Squares in Regressions with Integrated Regressors," Journal of the American Statistical Association, 83, 111-115.

[23] Summers, R., and Heston, A. (1991), "The Penn World Table; An Expanded Set of International Comparisons 1950-1988," Quarterly Journal of Economics, 106, 327-368.

[24] Wooldridge, J. (2002), Econometric Analysis of Cross Section and Panel Data, Cambridge, MIT Press. 


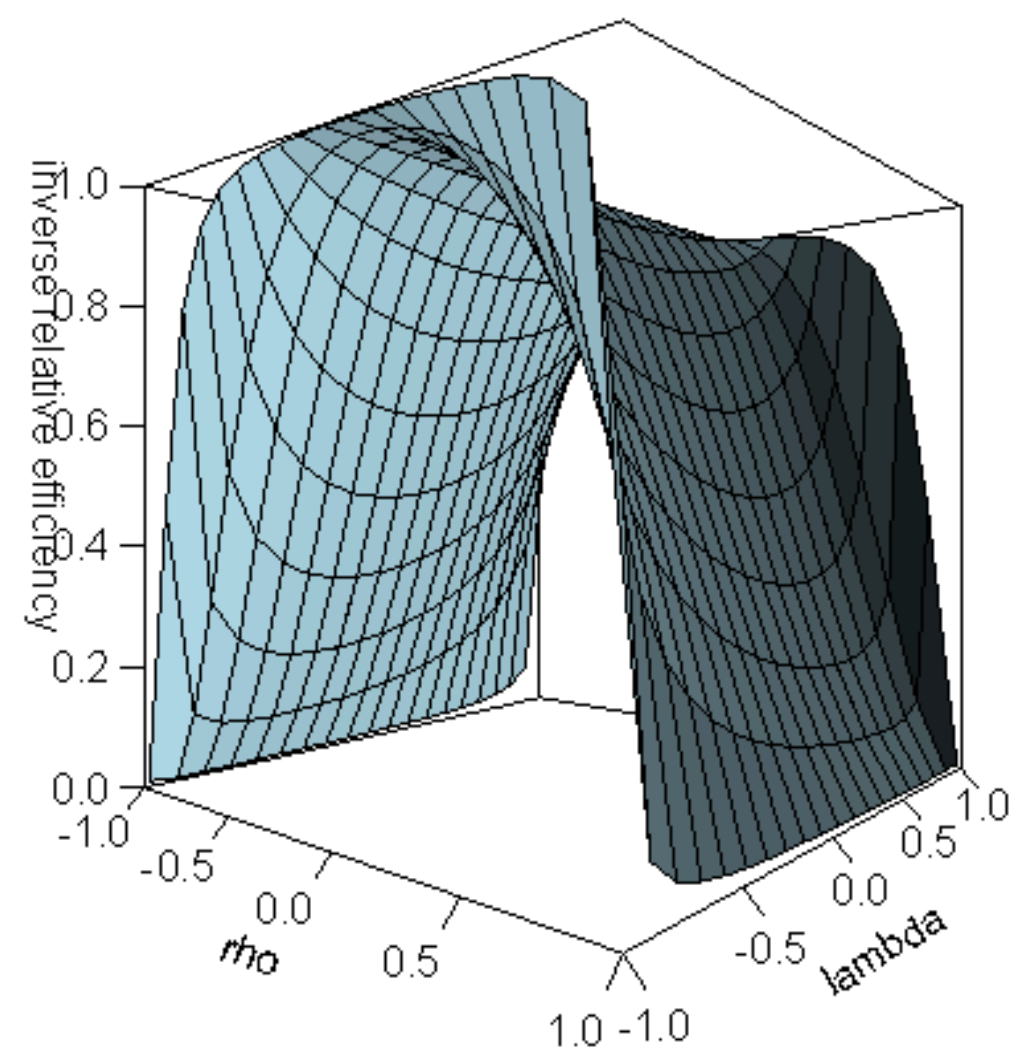

Figure 1: Relative Efficiency of GLS to FE Estimator 


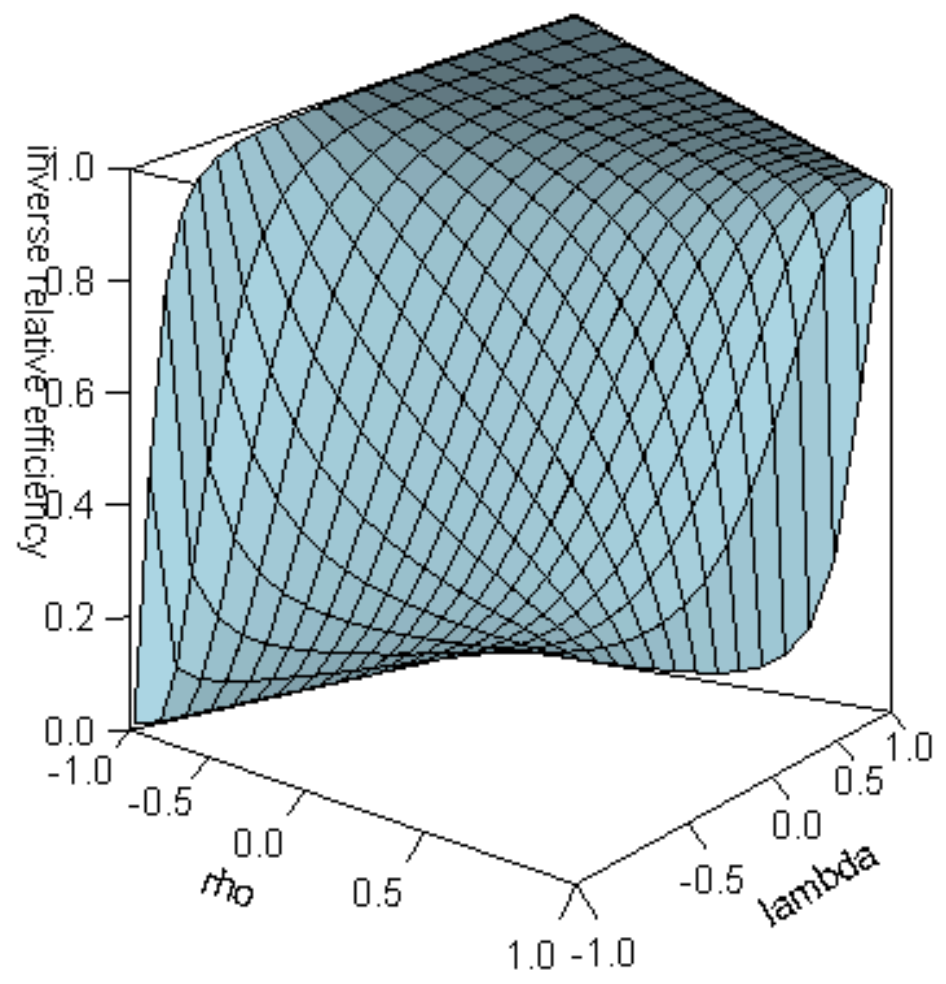

Figure 2: Relative Efficiency of GLS to FD Estimator 
Table 1: Relative Efficiencies of Standard Panel Data Estimators $(\mathrm{N}=40, \mathrm{~T}=20)$

\begin{tabular}{|c|c|c|c|c|c|c|c|c|}
\hline & \multirow[t]{2}{*}{$\rho$} & \multicolumn{7}{|c|}{$\lambda$} \\
\hline & & 0 & 0.2 & 0.4 & 0.6 & 0.8 & 0.9 & 1 \\
\hline \multirow[t]{7}{*}{ OLS } & 0 & 1.915 & 2.310 & 2.897 & 3.838 & 5.305 & 5.799 & 5.211 \\
\hline & 0.2 & 2.077 & 2.404 & 2.845 & 3.496 & 4.436 & 4.668 & 4.065 \\
\hline & 0.4 & 2.512 & 2.815 & 3.154 & 3.565 & 4.032 & 3.994 & 3.273 \\
\hline & 0.6 & 3.427 & 3.793 & 4.108 & 4.340 & 4.346 & 3.956 & 2.906 \\
\hline & 0.8 & 5.784 & 6.476 & 7.017 & 7.260 & 6.745 & 5.653 & 3.506 \\
\hline & 0.9 & 9.257 & 10.529 & 11.590 & 12.198 & 11.475 & 9.528 & 5.451 \\
\hline & 1 & 22.685 & 26.255 & 29.502 & 31.992 & 31.608 & 27.087 & 15.126 \\
\hline \multirow[t]{7}{*}{$\mathrm{FE}$} & 0 & 1.002 & 1.003 & 1.005 & 1.009 & 1.024 & 1.048 & 1.102 \\
\hline & 0.2 & 1.073 & 1.075 & 1.073 & 1.071 & 1.076 & 1.098 & 1.161 \\
\hline & 0.4 & 1.315 & 1.332 & 1.332 & 1.311 & 1.278 & 1.281 & 1.330 \\
\hline & 0.6 & 1.838 & 1.915 & 1.949 & 1.918 & 1.811 & 1.755 & 1.733 \\
\hline & 0.8 & 2.970 & 3.223 & 3.397 & 3.426 & 3.198 & 2.972 & 2.650 \\
\hline & 0.9 & 4.066 & 4.505 & 4.843 & 4.976 & 4.678 & 4.294 & 3.619 \\
\hline & 1 & 7.306 & 8.326 & 9.222 & 9.843 & 9.767 & 9.330 & 8.142 \\
\hline \multirow[t]{7}{*}{ FD } & 0 & 1.486 & 1.699 & 2.021 & 2.561 & 3.601 & 4.594 & 7.548 \\
\hline & 0.2 & 1.223 & 1.329 & 1.494 & 1.780 & 2.355 & 2.934 & 4.722 \\
\hline & 0.4 & 1.095 & 1.141 & 1.215 & 1.350 & 1.642 & 1.964 & 3.022 \\
\hline & 0.6 & 1.037 & 1.053 & 1.079 & 1.129 & 1.253 & 1.413 & 1.991 \\
\hline & 0.8 & 1.013 & 1.016 & 1.022 & 1.032 & 1.064 & 1.117 & 1.342 \\
\hline & 0.9 & 1.006 & 1.007 & 1.009 & 1.011 & 1.020 & 1.036 & 1.117 \\
\hline & 1 & 1.016 & 1.019 & 1.021 & 1.022 & 1.023 & 1.023 & 1.024 \\
\hline \multirow[t]{7}{*}{ GLS-PW } & 0 & 1.272 & 1.357 & 1.456 & 1.565 & 1.648 & 1.652 & 1.625 \\
\hline & 0.2 & 1.120 & 1.168 & 1.232 & 1.316 & 1.410 & 1.440 & 1.453 \\
\hline & 0.4 & 1.043 & 1.063 & 1.092 & 1.137 & 1.201 & 1.234 & 1.270 \\
\hline & 0.6 & 1.012 & 1.017 & 1.025 & 1.039 & 1.065 & 1.085 & 1.119 \\
\hline & 0.8 & 1.002 & 1.002 & 1.003 & 1.004 & 1.008 & 1.014 & 1.027 \\
\hline & 0.9 & 1.000 & 1.000 & 1.000 & 1.000 & 1.002 & 1.006 & 1.021 \\
\hline & 1 & 1.016 & 1.018 & 1.020 & 1.024 & 1.031 & 1.045 & 1.111 \\
\hline
\end{tabular}

Notes: (a) Relative mean square error with respect to the true GLS. (b) 10,000 replications. $\sigma_{\mu}^{2}=\sigma_{e}^{2}=5$. 
Table 2: Relative Efficiencies of Standard Panel Data Estimators $(\mathrm{N}=60, \mathrm{~T}=60)$

\begin{tabular}{|c|c|c|c|c|c|c|c|c|}
\hline & \multirow[t]{2}{*}{$\rho$} & \multicolumn{7}{|c|}{$\lambda$} \\
\hline & & 0 & 0.2 & 0.4 & 0.6 & 0.8 & 0.9 & 1 \\
\hline \multirow[t]{7}{*}{ OLS } & 0 & 1.963 & 2.414 & 3.123 & 4.441 & 7.826 & 12.201 & 13.958 \\
\hline & 0.2 & 2.101 & 2.467 & 2.989 & 3.881 & 6.062 & 8.836 & 9.628 \\
\hline & 0.4 & 2.523 & 2.858 & 3.249 & 3.796 & 4.980 & 6.446 & 6.329 \\
\hline & 0.6 & 3.445 & 3.848 & 4.207 & 4.509 & 4.844 & 5.209 & 4.077 \\
\hline & 0.8 & 6.008 & 6.802 & 7.438 & 7.754 & 7.290 & 6.375 & 3.150 \\
\hline & 0.9 & 10.598 & 12.223 & 13.622 & 14.515 & 13.850 & 11.702 & 4.367 \\
\hline & 1 & 61.715 & 73.063 & 84.083 & 94.300 & 100.976 & 97.379 & 41.327 \\
\hline \multirow[t]{7}{*}{$\mathrm{FE}$} & 0 & 1.000 & 1.000 & 1.000 & 1.000 & 1.002 & 1.005 & 1.037 \\
\hline & 0.2 & 1.081 & 1.083 & 1.078 & 1.064 & 1.039 & 1.028 & 1.062 \\
\hline & 0.4 & 1.359 & 1.379 & 1.371 & 1.323 & 1.219 & 1.151 & 1.149 \\
\hline & 0.6 & 2.010 & 2.110 & 2.147 & 2.073 & 1.802 & 1.576 & 1.415 \\
\hline & 0.8 & 3.871 & 4.291 & 4.601 & 4.681 & 4.180 & 3.485 & 2.511 \\
\hline & 0.9 & 6.686 & 7.647 & 8.490 & 9.050 & 8.675 & 7.464 & 4.664 \\
\hline & 1 & 20.601 & 24.289 & 27.902 & 31.303 & 33.476 & 32.129 & 23.459 \\
\hline \multirow[t]{7}{*}{ FD } & 0 & 1.485 & 1.714 & 2.079 & 2.779 & 4.672 & 7.440 & 21.119 \\
\hline & 0.2 & 1.211 & 1.322 & 1.505 & 1.864 & 2.861 & 4.347 & 11.967 \\
\hline & 0.4 & 1.082 & 1.128 & 1.206 & 1.367 & 1.834 & 2.559 & 6.559 \\
\hline & 0.6 & 1.026 & 1.041 & 1.067 & 1.121 & 1.292 & 1.579 & 3.449 \\
\hline & 0.8 & 1.007 & 1.009 & 1.014 & 1.023 & 1.056 & 1.123 & 1.768 \\
\hline & 0.9 & 1.002 & 1.003 & 1.004 & 1.007 & 1.014 & 1.030 & 1.267 \\
\hline & 1 & 1.006 & 1.007 & 1.008 & 1.009 & 1.010 & 1.010 & 1.010 \\
\hline \multirow[t]{7}{*}{ GLS-PW } & 0 & 1.293 & 1.392 & 1.512 & 1.660 & 1.820 & 1.834 & 1.667 \\
\hline & 0.2 & 1.126 & 1.181 & 1.258 & 1.376 & 1.564 & 1.663 & 1.612 \\
\hline & 0.4 & 1.043 & 1.066 & 1.101 & 1.162 & 1.291 & 1.399 & 1.455 \\
\hline & 0.6 & 1.011 & 1.017 & 1.026 & 1.045 & 1.095 & 1.155 & 1.246 \\
\hline & 0.8 & 1.001 & 1.002 & 1.003 & 1.005 & 1.010 & 1.021 & 1.062 \\
\hline & 0.9 & 1.000 & 1.000 & 1.000 & 1.000 & 1.001 & 1.002 & 1.012 \\
\hline & 1 & 1.005 & 1.006 & 1.007 & 1.008 & 1.009 & 1.010 & 1.046 \\
\hline
\end{tabular}

Notes: (a) Relative mean square error with respect to the true GLS. (b) 10,000 replications. $\sigma_{\mu}^{2}=\sigma_{e}^{2}=5$. 
Table 3: Relative Efficiencies of Standard Panel Data Estimators $(\mathrm{N}=240, \mathrm{~T}=60)$

\begin{tabular}{|c|c|c|c|c|c|c|c|c|}
\hline & \multirow[t]{2}{*}{$\rho$} & \multicolumn{7}{|c|}{$\lambda$} \\
\hline & & 0 & 0.2 & 0.4 & 0.6 & 0.8 & 0.9 & 1 \\
\hline \multirow[t]{7}{*}{ OLS } & 0 & 1.903 & 2.330 & 3.022 & 4.305 & 7.364 & 11.040 & 12.103 \\
\hline & 0.2 & 2.042 & 2.390 & 2.895 & 3.771 & 5.770 & 8.095 & 8.331 \\
\hline & 0.4 & 2.462 & 2.783 & 3.152 & 3.690 & 4.802 & 6.021 & 5.490 \\
\hline & 0.6 & 3.385 & 3.783 & 4.110 & 4.392 & 4.731 & 5.003 & 3.598 \\
\hline & 0.8 & 5.877 & 6.679 & 7.269 & 7.545 & 7.169 & 6.308 & 2.932 \\
\hline & 0.9 & 10.283 & 11.912 & 13.242 & 14.094 & 13.687 & 11.809 & 4.236 \\
\hline & 1 & 66.063 & 78.409 & 89.876 & 100.477 & 108.863 & 107.078 & 43.764 \\
\hline \multirow[t]{7}{*}{$\mathrm{FE}$} & 0 & 1.000 & 1.001 & 1.001 & 1.002 & 1.006 & 1.011 & 1.024 \\
\hline & 0.2 & 1.085 & 1.088 & 1.082 & 1.073 & 1.065 & 1.060 & 1.054 \\
\hline & 0.4 & 1.369 & 1.395 & 1.384 & 1.344 & 1.274 & 1.219 & 1.147 \\
\hline & 0.6 & 2.036 & 2.152 & 2.182 & 2.113 & 1.912 & 1.718 & 1.433 \\
\hline & 0.8 & 3.850 & 4.301 & 4.598 & 4.683 & 4.352 & 3.789 & 2.601 \\
\hline & 0.9 & 6.426 & 7.390 & 8.173 & 8.699 & 8.595 & 7.716 & 4.808 \\
\hline & 1 & 19.538 & 23.082 & 26.375 & 29.349 & 31.405 & 30.910 & 26.383 \\
\hline \multirow[t]{7}{*}{ FD } & 0 & 1.471 & 1.687 & 2.051 & 2.733 & 4.389 & 6.646 & 18.377 \\
\hline & 0.2 & 1.210 & 1.312 & 1.495 & 1.853 & 2.748 & 3.973 & 10.465 \\
\hline & 0.4 & 1.085 & 1.125 & 1.203 & 1.368 & 1.803 & 2.409 & 5.791 \\
\hline & 0.6 & 1.030 & 1.041 & 1.065 & 1.123 & 1.289 & 1.536 & 3.103 \\
\hline & 0.8 & 1.009 & 1.011 & 1.014 & 1.024 & 1.059 & 1.120 & 1.675 \\
\hline & 0.9 & 1.004 & 1.004 & 1.005 & 1.007 & 1.015 & 1.030 & 1.241 \\
\hline & 1 & 0.999 & 0.998 & 0.997 & 0.996 & 0.998 & 0.998 & 1.000 \\
\hline \multirow[t]{7}{*}{ GLS-PW } & 0 & 1.290 & 1.384 & 1.508 & 1.647 & 1.714 & 1.640 & 1.527 \\
\hline & 0.2 & 1.128 & 1.179 & 1.258 & 1.374 & 1.504 & 1.523 & 1.473 \\
\hline & 0.4 & 1.047 & 1.066 & 1.102 & 1.165 & 1.268 & 1.321 & 1.336 \\
\hline & 0.6 & 1.014 & 1.018 & 1.027 & 1.047 & 1.091 & 1.125 & 1.163 \\
\hline & 0.8 & 1.003 & 1.003 & 1.004 & 1.006 & 1.013 & 1.019 & 1.033 \\
\hline & 0.9 & 1.001 & 1.001 & 1.001 & 1.001 & 1.003 & 1.004 & 1.008 \\
\hline & 1 & 0.999 & 0.998 & 0.997 & 0.997 & 0.999 & 1.002 & 1.045 \\
\hline
\end{tabular}

Notes: (a) Relative mean square error with respect to the true GLS. (b) 10,000 replications. $\sigma_{\mu}^{2}=\sigma_{e}^{2}=5$. 


\section{Appendix}

\section{A Proof of Theorem 1}

The following lemmas are needed to prove Theorem 1. All limits are taken as $T \rightarrow \infty$ and followed by $n \rightarrow \infty$ sequentially, $(n, T) \stackrel{\text { seq }}{\rightarrow} \infty$.

Lemma 1 If Assumptions $1-2$ hold, then

1. If $|\lambda|<1, \frac{1}{n T} \sum_{i=1}^{n} \sum_{t=1}^{T}\left(x_{i t}-\overline{\bar{x}}\right)^{2} \stackrel{p}{\rightarrow} \frac{\sigma_{\varepsilon}^{2}}{1-\lambda^{2}}+\frac{2 \lambda \gamma_{\varepsilon}^{2}}{(1-\lambda)^{2}(1+\lambda)}$.

2. If $\lambda=1, \frac{1}{n T^{2}} \sum_{i=1}^{n} \sum_{t=1}^{T}\left(x_{i t}-\overline{\bar{x}}\right)^{2} \stackrel{p}{\rightarrow} \frac{\varpi_{\varepsilon}^{2}}{2}$.

Proof. Consider (1). For a fixed $n$, it is clear to see that

$$
\begin{aligned}
& \frac{1}{n T} \sum_{i=1}^{n} \sum_{t=1}^{T}\left(x_{i t}-\overline{\bar{x}}\right)^{2} \\
= & \frac{1}{n} \sum_{i=1}^{n}\left[\frac{1}{T} \sum_{t=1}^{T} x_{i t}^{2}\right]-(\overline{\bar{x}})^{2} \\
& \frac{p}{\rightarrow} \frac{1}{n} \sum_{i=1}^{n}\left[\frac{\sigma_{\varepsilon}^{2}}{1-\lambda^{2}}+\frac{2 \lambda \gamma_{\varepsilon}^{2}}{(1-\lambda)^{2}(1+\lambda)}\right]-\frac{1}{n} \sum_{i=1}^{n} E\left(x_{i t}\right) \\
= & \frac{\sigma_{\varepsilon}^{2}}{1-\lambda^{2}}+\frac{2 \lambda \gamma_{\varepsilon}^{2}}{(1-\lambda)^{2}(1+\lambda)}
\end{aligned}
$$

as $T \rightarrow \infty$ because

$$
\begin{aligned}
E\left(x_{i t}^{2}\right) & =E\left(\sum_{j=0}^{\infty} \lambda^{j} \varepsilon_{i(t-j)}\right)^{2} \\
& =\sum_{j=0}^{\infty} E\left(\lambda^{j} \varepsilon_{i(t-j)}\right)^{2}+\sum_{j=0}^{\infty} \sum_{k=0, k \neq j}^{\infty} E\left(\lambda^{j+k} \varepsilon_{i(t-j)} \varepsilon_{i(t-k)}\right) \\
& =\frac{\sigma_{\varepsilon}^{2}}{1-\lambda^{2}}+\frac{2 \lambda \gamma_{\varepsilon}^{2}}{(1-\lambda)^{2}(1+\lambda)}
\end{aligned}
$$

and $\overline{\bar{x}}=\frac{1}{n} \sum_{i=1}^{n} \frac{1}{T} \sum_{t=1}^{T} x_{i t} \stackrel{p}{\rightarrow} \frac{1}{n} \sum_{i=1}^{n} E\left(x_{i t}\right)=0$ as $T \rightarrow \infty$. Note $E\left(x_{i t}\right)=0$ for all $i$ and $t$ since there is no non-zero drift in (??). Then obviously,

$$
\frac{1}{n T} \sum_{i=1}^{n} \sum_{t=1}^{T}\left(x_{i t}-\overline{\bar{x}}\right)^{2} \stackrel{p}{\rightarrow} \frac{\sigma_{\varepsilon}^{2}}{1-\lambda^{2}}+\frac{2 \lambda \gamma_{\varepsilon}^{2}}{(1-\lambda)^{2}(1+\lambda)}
$$


holds for all $n$ and hence it holds for a large $n$ as well. This proves (1).

Next we consider (2). Similarly for a fixed $n$

$$
\begin{aligned}
& \frac{1}{n T^{2}} \sum_{i=1}^{n} \sum_{t=1}^{T}\left(x_{i t}-\overline{\bar{x}}\right)^{2} \\
= & \frac{1}{n} \sum_{i=1}^{n}\left[\frac{1}{T^{2}} \sum_{t=1}^{T} x_{i t}^{2}\right]-\frac{1}{T} \overline{\bar{x}}^{2} \\
= & \frac{1}{n} \sum_{i=1}^{n}\left[\frac{1}{T} \sum_{t=1}^{T}\left(\frac{x_{i t}}{\sqrt{T}}\right)^{2}\right]-\left[\frac{1}{n} \sum_{i=1}^{n}\left(\frac{1}{T} \sum_{t=1}^{T} \frac{x_{i t}}{\sqrt{T}}\right)\right]^{2} \\
\Rightarrow & \frac{1}{n} \sum_{i=1}^{n}\left(\varpi_{\varepsilon}^{2} \int W_{i}^{2}\right)-\left[\frac{1}{n} \sum_{i=1}^{n} \varpi_{\varepsilon} \int W_{i}\right]^{2}
\end{aligned}
$$

as $T \rightarrow \infty$ because $\frac{1}{T} \sum_{t=1}^{T}\left(\frac{x_{i t}}{\sqrt{T}}\right)^{2} \Rightarrow \varpi_{\varepsilon}^{2} \int W_{i}^{2}$ and $\frac{1}{T} \sum_{t=1}^{T} \frac{x_{i t}}{\sqrt{T}} \Rightarrow \varpi_{\varepsilon} \int W_{i}$.

Then

$$
\frac{1}{n} \sum_{i=1}^{n}\left(\varpi_{\varepsilon}^{2} \int W_{i}^{2}\right)-\left[\frac{1}{n} \sum_{i=1}^{n} \varpi_{\varepsilon} \int W_{i}\right]^{2} \stackrel{p}{\rightarrow} \frac{\varpi_{\varepsilon}^{2}}{2} .
$$

as $n \rightarrow \infty$ by a law of large numbers (LLN). This is because

$$
E \int W_{i}^{2}=\frac{1}{2}
$$

and

$$
E \int W_{i}=0
$$

This proves (2).

Lemma 2 If Assumptions $1-3$ hold, then

1. If $|\rho|<1$ and $|\lambda|<1$,

(a) $\frac{1}{n T} \sum_{i=1}^{n} \sum_{t=1}^{T}\left(x_{i t}-\overline{\bar{x}}\right) u_{i t} \stackrel{p}{\rightarrow} \lim \frac{1}{n T} \sum_{i=1}^{n} \sum_{t=1}^{T} E\left(x_{i t} \nu_{i t}\right)$

(b)

$$
\begin{aligned}
& \frac{1}{\sqrt{n T}} \sum_{i=1}^{n} \sum_{t=1}^{T}\left(x_{i t}-\overline{\bar{x}}\right) u_{i t}-\sqrt{n} \lim \frac{1}{n T} \sum_{i=1}^{n} \sum_{t=1}^{T} E\left(x_{i t} \nu_{i t}\right) \\
\Rightarrow & N\left(0, \frac{\sigma_{\mu}^{2} \varpi_{\varepsilon}^{2}}{(1-\lambda)^{2}}+\frac{\psi_{00}+\sum_{r=1}^{\infty} \lambda^{2 r} \psi_{0 r}+\sum_{r=1}^{\infty} \rho^{2 r} \psi_{r 0}}{(1-\rho \lambda)^{2}}\right)
\end{aligned}
$$




$$
\text { where } \psi_{0 r}=E\left(\varepsilon_{i(t-r)}^{2} e_{i t}^{2}\right), \psi_{r 0}=E\left(\varepsilon_{i t}^{2} e_{i(t-r)}^{2}\right), \psi_{00}=E\left(\varepsilon_{i t}^{2} e_{i t}^{2}\right) \text {. }
$$

2. If $\rho=1$ and $|\lambda|<1$,
(a) $\frac{1}{n T} \sum_{i=1}^{n} \sum_{t=1}^{T}\left(x_{i t}-\overline{\bar{x}}\right) u_{i t} \stackrel{p}{\rightarrow} \frac{-\frac{1}{2} \varpi_{e \varepsilon}+\delta_{e \varepsilon}}{1-\lambda}$
(b) $\frac{1}{\sqrt{n} T} \sum_{i=1}^{n} \sum_{t=1}^{T}\left(x_{i t}-\overline{\bar{x}}\right) u_{i t}-\sqrt{n}\left[\frac{\left(\frac{1}{n} \sum_{i=1}^{n} \frac{1}{T} \sum_{t=1}^{T} \varpi_{e \varepsilon} \nu_{i(t-1)} e_{i t}\right)+\delta_{e \varepsilon}}{1-\lambda}\right] \Rightarrow$ $N\left(0, \frac{\varpi_{\varepsilon, e} \varpi_{e}^{2}}{2(1-\lambda)^{2}}\right)$.

3. If $|\rho|<1$ and $\lambda=1$,
(a) $\frac{1}{n T^{3 / 2}} \sum_{i=1}^{n} \sum_{t=1}^{T}\left(x_{i t}-\overline{\bar{x}}\right) u_{i t} \stackrel{p}{\rightarrow} 0$
(b) $\frac{1}{\sqrt{n} T^{3 / 2}} \sum_{i=1}^{n} \sum_{t=1}^{T}\left(x_{i t}-\overline{\bar{x}}\right) u_{i t} \Rightarrow N\left(0, \frac{\sigma_{\mu}^{2} \varpi_{\varepsilon}^{2}}{3}\right)$.

4. If $\rho=1$ and $\lambda=1$,
(a) $\frac{1}{n T^{2}} \sum_{i=1}^{n} \sum_{t=1}^{T}\left(x_{i t}-\overline{\bar{x}}\right) u_{i t} \stackrel{p}{\rightarrow} \delta_{\varepsilon e}+\frac{\varpi_{\varepsilon e}}{2}$
(b) $\frac{1}{\sqrt{n} T^{2}} \sum_{i=1}^{n} \sum_{t=1}^{T}\left(x_{i t}-\overline{\bar{x}}\right) u_{i t}-\sqrt{n}\left[\left(\frac{1}{n} \sum_{i=1}^{n} \frac{1}{T} \sum_{t=1}^{T} x_{i(t-1)} \varepsilon_{i t}\right) \frac{\varpi_{\varepsilon e}}{\varpi_{\varepsilon}^{2}}+\delta_{\varepsilon e}\right] \Rightarrow$ $N\left(0, \frac{\varpi_{e . \varepsilon} \varpi_{\varepsilon}^{2}}{6}\right)$.

Proof. :

Consider (1). For part (a), we note

$$
\begin{aligned}
& \frac{1}{n T} \sum_{i=1}^{n} \sum_{t=1}^{T}\left(x_{i t}-\overline{\bar{x}}\right) u_{i t} \\
= & \frac{1}{n T} \sum_{i=1}^{n} \sum_{t=1}^{T}\left(x_{i t}-\overline{\bar{x}}\right)\left(\mu_{i}+\nu_{i t}\right) \\
= & \frac{1}{\sqrt{T}} \frac{1}{n} \sum_{i=1}^{n}\left[\left(\frac{1}{\sqrt{T}} \sum_{t=1}^{T} x_{i t}\right) \mu_{i}\right]-\frac{1}{\sqrt{T}}\left(\frac{1}{n} \sum_{i=1}^{n} \frac{1}{\sqrt{T}} \sum_{t=1}^{T} x_{i t}\right)\left(\frac{1}{n} \sum_{i=1}^{n} \mu_{i}\right) \\
& +\frac{1}{n} \sum_{i=1}^{n}\left[\frac{1}{T} \sum_{t=1}^{T} x_{i t} \nu_{i t}\right]-\left(\frac{1}{n} \sum_{i=1}^{n} \frac{1}{T} \sum_{t=1}^{T} x_{i t}\right)\left(\frac{1}{n} \sum_{i=1}^{n} \frac{1}{T} \sum_{t=1}^{T} \nu_{i t}\right) \\
= & I-I I+I I I-I V .
\end{aligned}
$$


Consider $I$. It is easy to see that for a fixed $n$,

$$
\frac{1}{n} \sum_{i=1}^{n}\left[\left(\frac{1}{\sqrt{T}} \sum_{t=1}^{T} x_{i t}\right) \mu_{i}\right]=\frac{1}{n} \sum_{i=1}^{n} Z_{i} \mu_{i}
$$

as $T \rightarrow \infty$ where

$$
\frac{1}{\sqrt{T}} \sum_{t=1}^{T} x_{i t} \Rightarrow Z_{i} \sim N\left(0, \frac{\varpi_{\varepsilon}^{2}}{(1-\lambda)^{2}}\right)
$$

by a central limit theorem (CLT) since $E\left(x_{i t}\right)=0$. Then

$$
\frac{1}{n} \sum_{i=1}^{n} Z_{i} \mu_{i}=o_{p}(1)
$$

as $n \rightarrow \infty$ by a LLN and the assumption that $\mu_{i}$ and $x_{i t}$ are uncorrected as in (??). Hence $I=\frac{1}{\sqrt{T}} o_{p}(1)$. Consider $I I$. For a fixed $n$,

$$
\begin{aligned}
& \left(\frac{1}{n} \sum_{i=1}^{n} \frac{1}{\sqrt{T}} \sum_{t=1}^{T} x_{i t}\right)\left(\frac{1}{n} \sum_{i=1}^{n} \mu_{i}\right) \\
= & \left(\frac{1}{n} \sum_{i=1}^{n} Z_{i}\right)\left(\frac{1}{n} \sum_{i=1}^{n} \mu_{i}\right)
\end{aligned}
$$

as $T \rightarrow \infty$. Then clearly

$$
\left(\frac{1}{n} \sum_{i=1}^{n} Z_{i}\right)\left(\frac{1}{n} \sum_{i=1}^{n} \mu_{i}\right) \stackrel{p}{\rightarrow} 0
$$

as $n \rightarrow \infty$ because $E\left(\mu_{i}\right)=0$. This proves that

$$
I I=\frac{1}{\sqrt{T}}\left(\frac{1}{n} \sum_{i=1}^{n} \frac{1}{\sqrt{T}} \sum_{t=1}^{T} x_{i t}\right)\left(\frac{1}{n} \sum_{i=1}^{n} \mu_{i}\right)=o_{p}(1) .
$$

Next we consider $I I I$. Clearly

$$
\frac{1}{n} \sum_{i=1}^{n}\left[\frac{1}{T} \sum_{t=1}^{T} x_{i t} \nu_{i t}\right]=\lim \frac{1}{n T} \sum_{i=1}^{n} \sum_{t=1}^{T} E\left(x_{i t} \nu_{i t}\right)+o_{p}(1)
$$

as $(n, T) \stackrel{\text { seq }}{\longrightarrow} \infty$. Also it is easy to see that

$$
I V=o_{p}(1)
$$

Collecting $I-I V$ we then prove (a). 
For part (b), for a fixed $n$,

$$
\begin{aligned}
& \frac{1}{\sqrt{n T}} \sum_{i=1}^{n} \sum_{t=1}^{T}\left(x_{i t}-\overline{\bar{x}}\right) u_{i t}-\sqrt{n T} \lim \frac{1}{n T} \sum_{i=1}^{n} \sum_{t=1}^{T} E\left(x_{i t} \nu_{i t}\right) \\
= & \frac{1}{\sqrt{n T}} \sum_{i=1}^{n} \sum_{t=1}^{T}\left(x_{i t}-\overline{\bar{x}}\right)\left(\mu_{i}+\nu_{i t}\right)-\sqrt{n T} \lim \frac{1}{n T} \sum_{i=1}^{n} \sum_{t=1}^{T} E\left(x_{i t} \nu_{i t}\right) \\
= & \frac{1}{\sqrt{n}} \sum_{i=1}^{n}\left[\left(\frac{1}{\sqrt{T}} \sum_{t=1}^{T} x_{i t}\right) \mu_{i}\right]-\left(\frac{1}{n} \sum_{i=1}^{n} \frac{1}{\sqrt{T}} \sum_{t=1}^{T} x_{i t}\right)\left(\frac{1}{\sqrt{n}} \sum_{i=1}^{n} \mu_{i}\right) \\
& +\left[\frac{1}{\sqrt{n}} \sum_{i=1}^{n}\left(\frac{1}{\sqrt{T}} \sum_{t=1}^{T} x_{i t} \nu_{i t}\right)-\sqrt{n T} \lim \frac{1}{n T} \sum_{i=1}^{n} \sum_{t=1}^{T} E\left(x_{i t} \nu_{i t}\right)\right] \\
& -\frac{1}{\sqrt{T}}\left(\frac{1}{n} \sum_{i=1}^{n} \frac{1}{\sqrt{T}} \sum_{t=1}^{T} x_{i t}\right)\left(\frac{1}{\sqrt{n}} \sum_{i=1}^{n} \frac{1}{\sqrt{T}} \sum_{t=1}^{T} \nu_{i t}\right) \\
= & I-I I+I I I-I V .
\end{aligned}
$$

For $I$,

$$
I=\frac{1}{\sqrt{n}} \sum_{i=1}^{n}\left[\left(\frac{1}{\sqrt{T}} \sum_{t=1}^{T} x_{i t}\right) \mu_{i}\right] \Rightarrow \frac{1}{\sqrt{n}} \sum_{i=1}^{n} Z_{i} \mu_{i}
$$

as $T \rightarrow \infty$, and

$$
\frac{1}{\sqrt{n}} \sum_{i=1}^{n} Z_{i} \mu_{i} \Rightarrow N\left(0, \frac{\sigma_{\mu}^{2} \varpi_{\varepsilon}^{2}}{(1-\lambda)^{2}}\right)
$$

as $n \rightarrow \infty$ by a CLT. For $I I I$,

$$
\begin{aligned}
& \frac{1}{\sqrt{n}} \sum_{i=1}^{n}\left(\frac{1}{\sqrt{T}} \sum_{t=1}^{T} x_{i t} \nu_{i t}\right)-\sqrt{n T} \lim \frac{1}{n T} \sum_{i=1}^{n} \sum_{t=1}^{T} E\left(x_{i t} \nu_{i t}\right) \\
= & \sqrt{n T} \frac{1}{n T} \sum_{i=1}^{n} \sum_{t=1}^{T}\left(x_{i t} \nu_{i t}-\lim \frac{1}{n T} \sum_{i=1}^{n} \sum_{t=1}^{T} E\left(x_{i t} \nu_{i t}\right)\right) \\
\Rightarrow & N\left(0, \frac{\psi_{i 00}+\sum_{r=1}^{\infty} \lambda^{2 r} \psi_{i 0 r}+\sum_{r=1}^{\infty} \rho^{2 r} \psi_{i r 0}}{(1-\rho \lambda)^{2}}\right)
\end{aligned}
$$

as $(n, T) \stackrel{\text { seq }}{\longrightarrow} \infty$, which is based on Lemma A0 in Choi(1999). It is easy to see that

$$
\begin{gathered}
I I=\left(\frac{1}{n} \sum_{i=1}^{n} \frac{1}{\sqrt{T}} \sum_{t=1}^{T} x_{i t}\right)\left(\frac{1}{\sqrt{n}} \sum_{i=1}^{n} \mu_{i}\right)=o_{p}(1) O_{p}(1) . \\
I V=\frac{1}{\sqrt{T}}\left(\frac{1}{n} \sum_{i=1}^{n} \frac{1}{\sqrt{T}} \sum_{t=1}^{T} x_{i t}\right)\left(\frac{1}{\sqrt{n}} \sum_{i=1}^{n} \frac{1}{\sqrt{T}} \sum_{t=1}^{T} \nu_{i t}\right)=\frac{1}{\sqrt{T}} o_{p}(1) O_{p}(1)=o_{p}(1) .
\end{gathered}
$$


Hence, we have

$$
\begin{aligned}
& \frac{1}{\sqrt{n T}} \sum_{i=1}^{n} \sum_{t=1}^{T}\left(x_{i t}-\overline{\bar{x}}\right) u_{i t}-\sqrt{n T} \lim \frac{1}{n T} \sum_{i=1}^{n} \sum_{t=1}^{T} E\left(x_{i t} \nu_{i t}\right) \\
\Rightarrow & N\left(0, \frac{\sigma_{\mu}^{2} \varpi_{\varepsilon}^{2}}{(1-\lambda)^{2}}+\frac{\psi_{00}+\sum_{r=1}^{\infty} \lambda^{2 r} \psi_{0 r}+\sum_{r=1}^{\infty} \rho^{2 r} \psi_{r 0}}{(1-\rho \lambda)^{2}}\right)
\end{aligned}
$$

as $(n, T) \stackrel{\text { seq }}{\rightarrow} \infty$. This proves $(\mathrm{b})$.

Consider (2). For part (a), note

$$
\begin{aligned}
& \frac{1}{n T} \sum_{i=1}^{n} \sum_{t=1}^{T}\left(x_{i t}-\overline{\bar{x}}\right) u_{i t} \\
= & \frac{1}{n T} \sum_{i=1}^{n} \sum_{t=1}^{T}\left(x_{i t}-\overline{\bar{x}}\right)\left(\mu_{i}+\nu_{i t}\right) \\
= & \frac{1}{\sqrt{T}} \frac{1}{n} \sum_{i=1}^{n}\left[\left(\frac{1}{\sqrt{T}} \sum_{t=1}^{T} x_{i t}\right) \mu_{i}\right]-\frac{1}{\sqrt{T}}\left(\frac{1}{n} \sum_{i=1}^{n} \frac{1}{\sqrt{T}} \sum_{t=1}^{T} x_{i t}\right)\left(\frac{1}{n} \sum_{i=1}^{n} \mu_{i}\right) \\
& +\frac{1}{n} \sum_{i=1}^{n}\left[\frac{1}{T} \sum_{t=1}^{T} x_{i t} \nu_{i t}\right]-\left(\frac{1}{n} \sum_{i=1}^{n} \frac{1}{\sqrt{T}} \sum_{t=1}^{T} x_{i t}\right)\left(\frac{1}{n} \sum_{i=1}^{n} \frac{1}{T} \sum_{t=1}^{T} \frac{\nu_{i t}}{\sqrt{T}}\right) \\
= & I-I I+I I I-I V .
\end{aligned}
$$

First we consider $I I I$. Note

$$
\frac{1}{T} \sum_{t=1}^{T} x_{i t} \nu_{i t} \Rightarrow \frac{1}{1-\lambda}\left[\varpi_{e} \varpi_{\varepsilon \cdot e}^{1 / 2}\left(\int V_{i} d W_{i}\right)+\varpi_{e \varepsilon}\left(\int V_{i} d V_{i}\right)+\delta_{e \varepsilon}\right]
$$

as $T \rightarrow \infty$. The above is taken from Lemma $1(\mathrm{a})$ in Kao and Chiang (2000).

Then

$$
\begin{aligned}
I I I= & \frac{1}{n} \sum_{i=1}^{n}\left[\frac{1}{T} \sum_{t=1}^{T} x_{i t} \nu_{i t}\right] \\
= & \frac{1}{n} \sum_{i=1}^{n} \frac{1}{1-\lambda}\left[\varpi_{e} \varpi_{\varepsilon \cdot e}^{1 / 2}\left(\int V_{i} d W_{i}\right)+\varpi_{e \varepsilon}\left(\int V_{i} d V_{i}\right)+\delta_{e \varepsilon}\right] \\
& \stackrel{p}{\rightarrow} \frac{-\frac{1}{2} \varpi_{e \varepsilon}+\delta_{e \varepsilon}}{1-\lambda}
\end{aligned}
$$

as $n \rightarrow \infty$ because $E\left(\int V_{i} d W_{i}\right)=0$ and $E\left(\int V_{i} d V_{i}\right)=-\frac{1}{2}$. It is clear to see that

$$
I=\frac{1}{\sqrt{T}} \frac{1}{n} \sum_{i=1}^{n}\left[\left(\frac{1}{\sqrt{T}} \sum_{t=1}^{T} x_{i t}\right) \mu_{i}\right]=\frac{1}{\sqrt{T}} o_{p}(1)
$$




$$
I I=\frac{1}{\sqrt{T}}\left(\frac{1}{n} \sum_{i=1}^{n} \frac{1}{\sqrt{T}} \sum_{t=1}^{T} x_{i t}\right)\left(\frac{1}{n} \sum_{i=1}^{n} \mu_{i}\right)=\frac{1}{\sqrt{T}} o_{p}(1)
$$

and

$$
I V=\left(\frac{1}{n} \sum_{i=1}^{n} \frac{1}{\sqrt{T}} \sum_{t=1}^{T} x_{i t}\right)\left(\frac{1}{n} \sum_{i=1}^{n} \frac{1}{T} \sum_{t=1}^{T} \frac{\nu_{i t}}{\sqrt{T}}\right)=o_{p}(1)
$$

because $\frac{1}{T^{3 / 2}} \sum_{t=1}^{T} \nu_{i t} \Rightarrow \int V_{i}$ and $E\left(\int V_{i}\right)=0$. This proves (a).

For part (b),

$$
\begin{aligned}
& \frac{1}{\sqrt{n} T} \sum_{i=1}^{n} \sum_{t=1}^{T}\left(x_{i t}-\overline{\bar{x}}\right) u_{i t} \\
= & \frac{1}{\sqrt{n} T} \sum_{i=1}^{n} \sum_{t=1}^{T}\left(x_{i t}-\overline{\bar{x}}\right)\left(\mu_{i}+\nu_{i t}\right) \\
= & \frac{1}{\sqrt{T}} \frac{1}{\sqrt{n}} \sum_{i=1}^{n}\left[\left(\frac{1}{\sqrt{T}} \sum_{t=1}^{T} x_{i t}\right) \mu_{i}\right]-\frac{1}{\sqrt{T}}\left(\frac{1}{n} \sum_{i=1}^{n} \frac{1}{\sqrt{T}} \sum_{t=1}^{T} x_{i t}\right)\left(\frac{1}{\sqrt{n}} \sum_{i=1}^{n} \mu_{i}\right) \\
& +\frac{1}{\sqrt{n}} \sum_{i=1}^{n}\left[\frac{1}{T} \sum_{t=1}^{T} x_{i t} \nu_{i t}\right]-\left(\frac{1}{n} \sum_{i=1}^{n} \frac{1}{\sqrt{T}} \sum_{t=1}^{T} x_{i t}\right)\left(\frac{1}{\sqrt{n}} \sum_{i=1}^{n} \frac{1}{T^{3 / 2}} \sum_{t=1}^{T} \nu_{i t}\right) \\
= & I-I I+I I I-I V .
\end{aligned}
$$

First consider $I I I$,

$$
\begin{aligned}
& \frac{1}{\sqrt{n}} \sum_{i=1}^{n}\left[\frac{1}{T} \sum_{t=1}^{T} x_{i t} \nu_{i t}\right]-\sqrt{n}\left[\frac{\left(\frac{1}{n} \sum_{i=1}^{n} \frac{1}{T} \sum_{t=1}^{T} \varpi_{e \varepsilon} \nu_{i, t-1} e_{i t}\right)+\delta_{e \varepsilon}}{1-\lambda}\right] \\
= & \frac{1}{\sqrt{n}} \sum_{i=1}^{n} \frac{1}{1-\lambda}\left[\varpi_{e} \varpi_{\varepsilon \cdot e}^{1 / 2}\left(\int V_{i} d W_{i}\right)+\varpi_{e \varepsilon}\left(\int V_{i} d V_{i}\right)+\delta_{e \varepsilon}\right] \\
& -\sqrt{n}\left[\frac{\frac{1}{n} \sum_{i=1}^{n}\left(\varpi_{e \varepsilon} \int V_{i} d V_{i}\right)+\delta_{e \varepsilon}}{1-\lambda}\right]+o_{p}(1) \\
= & \frac{1}{\sqrt{n}} \sum_{i=1}^{n} \frac{1}{1-\lambda}\left[\varpi_{e} \varpi_{\varepsilon \cdot e}^{1 / 2}\left(\int V_{i} d W_{i}\right)\right]+o_{p}(1) \\
\Rightarrow & N\left(0, \frac{\varpi_{\varepsilon \cdot e} \varpi_{e}^{2}}{2(1-\lambda)^{2}}\right),
\end{aligned}
$$

as $(n, T) \stackrel{\text { seq }}{\longrightarrow} \infty$. It is easy to see that

$$
I=\frac{1}{\sqrt{T}} \frac{1}{\sqrt{n}} \sum_{i=1}^{n}\left[\left(\frac{1}{\sqrt{T}} \sum_{t=1}^{T} x_{i t}\right) \mu_{i}\right]=\frac{1}{\sqrt{T}} O_{p}(1),
$$




$$
I I=\frac{1}{\sqrt{T}}\left(\frac{1}{n} \sum_{i=1}^{n} \frac{1}{\sqrt{T}} \sum_{t=1}^{T} x_{i t}\right)\left(\frac{1}{\sqrt{n}} \sum_{i=1}^{n} \mu_{i}\right)=\frac{1}{\sqrt{T}} o_{p}(1) O_{p}(1)
$$

and

$$
I V=\left(\frac{1}{n} \sum_{i=1}^{n} \frac{1}{\sqrt{T}} \sum_{t=1}^{T} x_{i t}\right)\left(\frac{1}{\sqrt{n}} \sum_{i=1}^{n} \frac{1}{T^{3 / 2}} \sum_{t=1}^{T} \nu_{i t}\right)=o_{p}(1) O_{p}(1) .
$$

Hence, we have

$$
\begin{aligned}
& \frac{1}{\sqrt{n} T} \sum_{i=1}^{n} \sum_{t=1}^{T}\left(x_{i t}-\overline{\bar{x}}\right) u_{i t}-\sqrt{n}\left[\frac{\left(\frac{1}{n} \sum_{i=1}^{n} \frac{1}{T} \sum_{t=1}^{T} \varpi_{e \varepsilon} \nu_{i(t-1)} e_{i t}\right)+\delta_{e \varepsilon}}{1-\lambda}\right] \\
\Rightarrow & N\left(0, \frac{\varpi_{\varepsilon . e} \varpi_{e}^{2}}{2(1-\lambda)^{2}}\right),
\end{aligned}
$$

as $(n, T) \stackrel{\text { seq }}{\longrightarrow} \infty$.

Consider (3). For part (a), it is easy to see that

$$
\begin{aligned}
& \frac{1}{n T^{3 / 2}} \sum_{i=1}^{n} \sum_{t=1}^{T}\left(x_{i t}-\overline{\bar{x}}\right) u_{i t} \\
= & \frac{1}{n T^{3 / 2}} \sum_{i=1}^{n} \sum_{t=1}^{T}\left(x_{i t}-\overline{\bar{x}}\right)\left(\mu_{i}+\nu_{i t}\right) \\
= & \frac{1}{n} \sum_{i=1}^{n}\left[\left(\frac{1}{T^{3 / 2}} \sum_{t=1}^{T} x_{i t}\right) \mu_{i}\right]-\left(\frac{1}{n} \sum_{i=1}^{n} \frac{1}{T^{3 / 2}} \sum_{t=1}^{T} x_{i t}\right)\left(\frac{1}{n} \sum_{i=1}^{n} \mu_{i}\right) \\
& +\frac{1}{\sqrt{T}} \frac{1}{n} \sum_{i=1}^{n}\left[\left(\frac{1}{T} \sum_{t=1}^{T} x_{i t} \nu_{i t}\right)\right]-\frac{1}{\sqrt{T}}\left(\frac{1}{n} \sum_{i=1}^{n} \frac{1}{T^{3 / 2}} \sum_{t=1}^{T} x_{i t}\right)\left(\frac{1}{n} \sum_{i=1}^{n} \frac{1}{\sqrt{T}} \sum_{t=1}^{T} \nu_{i t}\right) \\
= & I-I I+I I I-I V .
\end{aligned}
$$

For a fixed $n$,

$$
\begin{aligned}
I & =\frac{1}{n} \sum_{i=1}^{n}\left[\left(\frac{1}{T^{3 / 2}} \sum_{t=1}^{T} x_{i t}\right) \mu_{i}\right] \\
& \Rightarrow \frac{1}{n} \sum_{i=1}^{n}\left[\left(\varpi_{\varepsilon} \int W_{i}\right) \mu_{i}\right]
\end{aligned}
$$

as $T \rightarrow \infty$ by a CLT. As $n \rightarrow \infty$ by a LLN and the assumption that $\mu_{i}$ and $x_{i t}$ are uncorrected as in (??), we have $I=o_{p}(1)$. It is easy to show that

$$
I I=\left(\frac{1}{n} \sum_{i=1}^{n} \frac{1}{T^{3 / 2}} \sum_{t=1}^{T} x_{i t}\right)\left(\frac{1}{n} \sum_{i=1}^{n} \mu_{i}\right)=o_{p}(1) O_{p}(1),
$$




$$
I I I=\frac{1}{\sqrt{T}} \frac{1}{n} \sum_{i=1}^{n}\left[\left(\frac{1}{T} \sum_{t=1}^{T} x_{i t} \nu_{i t}\right)\right]=\frac{1}{\sqrt{T}} o_{p}(1)
$$

and

$$
I V=\frac{1}{\sqrt{T}}\left(\frac{1}{n} \sum_{i=1}^{n} \frac{1}{T^{3 / 2}} \sum_{t=1}^{T} x_{i t}\right)\left(\frac{1}{n} \sum_{i=1}^{n} \frac{1}{\sqrt{T}} \sum_{t=1}^{T} \nu_{i t}\right)=\frac{1}{\sqrt{T}} o_{p}(1) o_{p}(1)
$$

as $(n, T) \stackrel{\text { seq }}{\rightarrow} \infty$ because $\frac{1}{T^{3 / 2}} \sum_{t=1}^{T} x_{i t} \Rightarrow \varpi_{\varepsilon} \int W_{i}, E\left[\varpi_{\varepsilon} \int W_{i}\right]=0, E\left[\mu_{i}\right]=0$, $\frac{1}{T} \sum_{t=1}^{T} x_{i t} \nu_{i t} \Rightarrow \varpi_{\varepsilon} \varpi_{e} \int W_{i} d V_{i}, E\left[\varpi_{\varepsilon} \varpi_{e} \int W_{i} d V_{i}\right]=0$, and $\frac{1}{\sqrt{T}} \sum_{t=1}^{T} \nu_{i t} \Rightarrow$ $N\left(0, \frac{\varpi_{e}^{2}}{(1-\rho)^{2}}\right)$. Hence, we have

$$
\frac{1}{n T^{3 / 2}} \sum_{i=1}^{n} \sum_{t=1}^{T}\left(x_{i t}-\overline{\bar{x}}\right) u_{i t} \stackrel{p}{\rightarrow} 0
$$

as $(n, T) \stackrel{\text { seq }}{\rightarrow} \infty$.

For part (b), note that

$$
\begin{aligned}
& \frac{1}{n^{1 / 2} T^{3 / 2}} \sum_{i=1}^{n} \sum_{t=1}^{T}\left(x_{i t}-\overline{\bar{x}}\right) u_{i t} \\
= & \frac{1}{n^{1 / 2} T^{3 / 2}} \sum_{i=1}^{n} \sum_{t=1}^{T}\left(x_{i t}-\overline{\bar{x}}\right)\left(\mu_{i}+\nu_{i t}\right) \\
= & \frac{1}{\sqrt{n}} \sum_{i=1}^{n}\left[\left(\frac{1}{T^{3 / 2}} \sum_{t=1}^{T} x_{i t}\right) \mu_{i}\right]-\left(\frac{1}{n} \sum_{i=1}^{n} \frac{1}{T^{3 / 2}} \sum_{t=1}^{T} x_{i t}\right)\left(\frac{1}{\sqrt{n}} \sum_{i=1}^{n} \mu_{i}\right) \\
& +\frac{1}{\sqrt{T}} \frac{1}{\sqrt{n}} \sum_{i=1}^{n}\left[\frac{1}{T} \sum_{t=1}^{T} x_{i t} \nu_{i t}\right]-\frac{1}{\sqrt{T}}\left(\frac{1}{n} \sum_{i=1}^{n} \frac{1}{T^{3 / 2}} \sum_{t=1}^{T} x_{i t}\right)\left(\frac{1}{\sqrt{n}} \sum_{i=1}^{n} \frac{1}{\sqrt{T}} \sum_{t=1}^{T} \nu_{i t}\right) \\
= & I-I I+I I I-I V .
\end{aligned}
$$

For a fixed $n$,

$$
\begin{aligned}
I & =\frac{1}{\sqrt{n}} \sum_{i=1}^{n}\left[\left(\frac{1}{T^{3 / 2}} \sum_{t=1}^{T} x_{i t}\right) \mu_{i}\right] \\
& \Rightarrow \frac{1}{\sqrt{n}} \sum_{i=1}^{n}\left[\left(\varpi_{\varepsilon} \int W_{i}\right) \mu_{i}\right]
\end{aligned}
$$

as $T \rightarrow \infty$, and

$$
\frac{1}{\sqrt{n}} \sum_{i=1}^{n}\left[\left(\varpi_{\varepsilon} \int W_{i}\right) \mu_{i}\right] \Rightarrow N\left(0, \frac{\sigma_{\mu}^{2} \varpi_{\varepsilon}^{2}}{3}\right)
$$


as $n \rightarrow \infty$ by a CLT with $E\left[\varpi_{\varepsilon} \int W_{i}\right]=0$ and $\operatorname{Var}\left[\varpi_{\varepsilon} \int W_{i}\right]=\frac{1}{3} \varpi_{\varepsilon}^{2}$. It is easy to show that

$$
\begin{gathered}
I I=\left(\frac{1}{n} \sum_{i=1}^{n} \frac{1}{T^{3 / 2}} \sum_{t=1}^{T} x_{i t}\right)\left(\frac{1}{\sqrt{n}} \sum_{i=1}^{n} \mu_{i}\right)=o_{p}(1) O_{p}(1), \\
I I I=\frac{1}{\sqrt{T}} \frac{1}{\sqrt{n}} \sum_{i=1}^{n}\left[\frac{1}{T} \sum_{t=1}^{T} x_{i t} \nu_{i t}\right]=\frac{1}{\sqrt{T}} O_{p}(1)
\end{gathered}
$$

and

$$
I V=\frac{1}{\sqrt{T}}\left(\frac{1}{n} \sum_{i=1}^{n} \frac{1}{T^{3 / 2}} \sum_{t=1}^{T} x_{i t}\right)\left(\frac{1}{\sqrt{n}} \sum_{i=1}^{n} \frac{1}{\sqrt{T}} \sum_{t=1}^{T} \nu_{i t}\right)=\frac{1}{\sqrt{T}} o_{p}(1) O_{p}(1)
$$

as $(n, T) \stackrel{\text { seq }}{\longrightarrow} \infty$. Hence, we have

$$
\frac{1}{n^{1 / 2} T^{3 / 2}} \sum_{i=1}^{n} \sum_{t=1}^{T}\left(x_{i t}-\overline{\bar{x}}\right) u_{i t} \Rightarrow N\left(0, \frac{\sigma_{\mu}^{2} \varpi_{\varepsilon}^{2}}{3}\right)
$$

as $(n, T) \stackrel{\text { seq }}{\longrightarrow} \infty$.

Consider (4). For part (a), it is easy to see that

$$
\begin{aligned}
& \frac{1}{n T^{2}} \sum_{i=1}^{n} \sum_{t=1}^{T}\left(x_{i t}-\overline{\bar{x}}\right) u_{i t} \\
= & \frac{1}{n T^{2}} \sum_{i=1}^{n} \sum_{t=1}^{T}\left(x_{i t}-\overline{\bar{x}}\right)\left(\mu_{i}+\nu_{i t}\right) \\
= & \frac{1}{\sqrt{T}} \frac{1}{n} \sum_{i=1}^{n}\left[\left(\frac{1}{T^{3 / 2}} \sum_{t=1}^{T} x_{i t}\right) \mu_{i}\right]-\frac{1}{\sqrt{T}}\left(\frac{1}{n} \sum_{i=1}^{n} \frac{1}{T^{3 / 2}} \sum_{t=1}^{T} x_{i t}\right)\left(\frac{1}{n} \sum_{i=1}^{n} \mu_{i}\right) \\
& +\frac{1}{n} \sum_{i=1}^{n}\left[\frac{1}{T^{2}} \sum_{t=1}^{T} x_{i t} \nu_{i t}\right]-\left(\frac{1}{n} \sum_{i=1}^{n} \frac{1}{T^{3 / 2}} \sum_{t=1}^{T} x_{i t}\right)\left(\frac{1}{n} \sum_{i=1}^{n} \frac{1}{T^{3 / 2}} \sum_{t=1}^{T} \nu_{i t}\right) \\
= & I-I I+I I I-I V .
\end{aligned}
$$

For a fixed $n$,

$$
\begin{aligned}
I I I & =\frac{1}{n} \sum_{i=1}^{n}\left[\frac{1}{T^{2}} \sum_{t=1}^{T} x_{i t} \nu_{i t}\right] \\
& \Rightarrow \frac{1}{n} \sum_{i=1}^{n}\left[\varpi_{\varepsilon} \varpi_{e . \varepsilon}^{1 / 2} \int W_{i} V_{i}+\varpi_{\varepsilon e}\left(\int W_{i}^{2}\right)+\delta_{\varepsilon e}\right]
\end{aligned}
$$


as $T \rightarrow \infty$ by a CLT. We then have

$$
\frac{1}{n} \sum_{i=1}^{n}\left[\varpi_{\varepsilon} \varpi_{e . \varepsilon}^{1 / 2} \int W_{i} V_{i}+\varpi_{\varepsilon e}\left(\int W_{i}^{2}\right)+\delta_{\varepsilon e}\right] \stackrel{p}{\rightarrow} \delta_{\varepsilon e}+\frac{\varpi_{\varepsilon e}}{2} .
$$

as $n \rightarrow \infty$. So

$$
I I I \stackrel{p}{\rightarrow} \delta_{\varepsilon e}+\frac{\varpi_{\varepsilon e}}{2}
$$

as $(n, T) \stackrel{\text { seq }}{\longrightarrow} \infty$. Again

$$
\begin{gathered}
I=\frac{1}{\sqrt{T}} \frac{1}{n} \sum_{i=1}^{n}\left[\left(\frac{1}{T^{3 / 2}} \sum_{t=1}^{T} x_{i t}\right) \mu_{i}\right]=\frac{1}{\sqrt{T}} o_{p}(1) \\
I I=\frac{1}{\sqrt{T}}\left(\frac{1}{n} \sum_{i=1}^{n} \frac{1}{T^{3 / 2}} \sum_{t=1}^{T} x_{i t}\right)\left(\frac{1}{n} \sum_{i=1}^{n} \mu_{i}\right)=\frac{1}{\sqrt{T}} o_{p}(1) o_{p}(1)
\end{gathered}
$$

and

$$
I V=\left(\frac{1}{n} \sum_{i=1}^{n} \frac{1}{T^{3 / 2}} \sum_{t=1}^{T} x_{i t}\right)\left(\frac{1}{n} \sum_{i=1}^{n} \frac{1}{T^{3 / 2}} \sum_{t=1}^{T} \nu_{i t}\right)=o_{p}(1) o_{p}(1)
$$

as $(n, T) \stackrel{\text { seq }}{\rightarrow} \infty$. Hence, we have

$$
\frac{1}{n T^{2}} \sum_{i=1}^{n} \sum_{t=1}^{T}\left(x_{i t}-\overline{\bar{x}}\right) u_{i t} \stackrel{p}{\rightarrow} \delta_{\varepsilon e}+\frac{\varpi_{\varepsilon e}}{2}
$$

as $(n, T) \stackrel{\text { seq }}{\rightarrow} \infty$.

For part (b),

$$
\begin{aligned}
& \frac{1}{\sqrt{n} T^{2}} \sum_{i=1}^{n} \sum_{t=1}^{T}\left(x_{i t}-\overline{\bar{x}}\right) u_{i t} \\
= & \frac{1}{\sqrt{n} T^{2}} \sum_{i=1}^{n} \sum_{t=1}^{T}\left(x_{i t}-\overline{\bar{x}}\right)\left(\mu_{i}+\nu_{i t}\right) \\
= & \frac{1}{\sqrt{T}} \frac{1}{\sqrt{n}} \sum_{i=1}^{n}\left[\left(\frac{1}{T^{3 / 2}} \sum_{t=1}^{T} x_{i t}\right) \mu_{i}\right]-\frac{1}{\sqrt{T}}\left(\frac{1}{n} \sum_{i=1}^{n} \frac{1}{T^{3 / 2}} \sum_{t=1}^{T} x_{i t}\right)\left(\frac{1}{\sqrt{n}} \sum_{i=1}^{n} \mu_{i}\right) \\
& +\frac{1}{\sqrt{n}} \sum_{i=1}^{n} \frac{1}{T^{2}} \sum_{t=1}^{T} x_{i t} \nu_{i t}-\left(\frac{1}{n} \sum_{i=1}^{n} \frac{1}{T^{3 / 2}} \sum_{t=1}^{T} x_{i t}\right)\left(\frac{1}{\sqrt{n}} \sum_{i=1}^{n} \frac{1}{T^{3 / 2}} \sum_{t=1}^{T} \nu_{i t}\right) \\
= & I-I I+I I I-I V .
\end{aligned}
$$


For a fixed $n$,

$$
\begin{aligned}
I I I & =\frac{1}{\sqrt{n}} \sum_{i=1}^{n} \frac{1}{T^{2}} \sum_{t=1}^{T} x_{i t} \nu_{i t} \\
& \Rightarrow \frac{1}{\sqrt{n}} \sum_{i=1}^{n}\left[\varpi_{\varepsilon} \varpi_{e . \varepsilon}^{1 / 2} \int W_{i} V_{i}+\varpi_{\varepsilon e}\left(\int W_{i}^{2}\right)+\delta_{\varepsilon e}\right]
\end{aligned}
$$

as $T \rightarrow \infty$ by a CLT. As $(n, T) \stackrel{\text { seq }}{\rightarrow} \infty$, we have

$$
\begin{aligned}
& \frac{1}{\sqrt{n}} \sum_{i=1}^{n} \frac{1}{T^{2}} \sum_{t=1}^{T} x_{i t} \nu_{i t}-\sqrt{n}\left[\left(\frac{1}{n} \sum_{i=1}^{n} \frac{1}{T} \sum_{t=1}^{T} x_{i(t-1)} \varepsilon_{i t}\right) \frac{\varpi_{\varepsilon e}}{\varpi_{\varepsilon}^{2}}+\delta_{\varepsilon e}\right] \\
\Rightarrow & \frac{1}{\sqrt{n}} \sum_{i=1}^{n}\left[\varpi_{\varepsilon} \varpi_{e . \varepsilon}^{1 / 2} \int W_{i} V_{i}+\varpi_{\varepsilon e}\left(\int W_{i}^{2}\right)+\delta_{\varepsilon e}\right]-\frac{1}{\sqrt{n}} \sum_{i=1}^{n}\left[\varpi_{\varepsilon e}\left(\int W_{i}^{2}\right)+\delta_{\varepsilon e}\right] \\
= & \frac{1}{\sqrt{n}} \sum_{i=1}^{n}\left[\varpi_{\varepsilon} \varpi_{e . \varepsilon}^{1 / 2} \int W_{i} V_{i}\right] \\
\Rightarrow & N\left(0, \frac{\varpi_{e . \varepsilon} \varpi_{\varepsilon}^{2}}{6}\right) .
\end{aligned}
$$

Also it is easy to see that

$$
\begin{gathered}
I=\frac{1}{\sqrt{T}} \frac{1}{\sqrt{n}} \sum_{i=1}^{n}\left[\left(\frac{1}{T^{3 / 2}} \sum_{t=1}^{T} x_{i t}\right) \mu_{i}\right]=\frac{1}{\sqrt{T}} O_{p}(1), \\
I I=\frac{1}{\sqrt{T}}\left(\frac{1}{n} \sum_{i=1}^{n} \frac{1}{T^{3 / 2}} \sum_{t=1}^{T} x_{i t}\right)\left(\frac{1}{\sqrt{n}} \sum_{i=1}^{n} \mu_{i}\right)=\frac{1}{\sqrt{T}} o_{p}(1) O_{p}(1)
\end{gathered}
$$

and

$$
I V=\left(\frac{1}{n} \sum_{i=1}^{n} \frac{1}{T^{3 / 2}} \sum_{t=1}^{T} x_{i t}\right)\left(\frac{1}{\sqrt{n}} \sum_{i=1}^{n} \frac{1}{T^{3 / 2}} \sum_{t=1}^{T} \nu_{i t}\right)=o_{p}(1) O_{p}(1)
$$

as $(n, T) \stackrel{\text { seq }}{\rightarrow} \infty$. Hence, we have

$$
\begin{aligned}
& \frac{1}{\sqrt{n} T^{2}} \sum_{i=1}^{n} \sum_{t=1}^{T}\left(x_{i t}-\overline{\bar{x}}\right) u_{i t}-\sqrt{n}\left[\left(\frac{1}{n} \sum_{i=1}^{n} \frac{1}{T} \sum_{t=1}^{T} x_{i(t-1)} \varepsilon_{i t}\right) \frac{\varpi_{\varepsilon e}}{\varpi_{\varepsilon}^{2}}+\delta_{\varepsilon e}\right] \Rightarrow N\left(0, \frac{\varpi_{e . \varepsilon} \varpi_{\varepsilon}^{2}}{6}\right), \\
& \text { as }(n, T) \stackrel{\text { seq }}{\rightarrow} \infty \text {. }
\end{aligned}
$$

\section{B Proof of Theorem 1}

Proof. The proof is straightforward by using lemmas 1 and 2 . 


\section{Proof of Theorem 2}

The following lemmas will be used to prove Theorem 2 .

Lemma 3 If Assumptions $1-2$ hold, then

1. If $|\lambda|<1, \frac{1}{n T} \sum_{i=1}^{n} \sum_{t=1}^{T}\left(x_{i t}-\bar{x}_{i}\right)^{2} \stackrel{p}{\rightarrow} \frac{\sigma_{\varepsilon}^{2}}{1-\lambda^{2}}+\frac{2 \lambda \gamma_{\varepsilon}^{2}}{(1-\lambda)^{2}(1+\lambda)}$.

2. If $\lambda=1, \frac{1}{n T^{2}} \sum_{i=1}^{n} \sum_{t=1}^{T}\left(x_{i t}-\bar{x}_{i}\right)^{2} \stackrel{p}{\rightarrow} \frac{\varpi_{\varepsilon}^{2}}{6}$.

Proof. Consider (1). For a fixed $n$, it is clear to see that

$$
\begin{aligned}
& \frac{1}{n T} \sum_{i=1}^{n} \sum_{t=1}^{T}\left(x_{i t}-\bar{x}_{i}\right)^{2} \\
= & \frac{1}{n} \sum_{i=1}^{n}\left[\frac{1}{T} \sum_{t=1}^{T} x_{i t}^{2}\right]-\frac{1}{n} \sum_{i=1}^{n}\left[\bar{x}_{i}^{2}\right] \\
& \frac{p}{\rightarrow} \frac{1}{n} \sum_{i=1}^{n}\left[\frac{\sigma_{\varepsilon}^{2}}{1-\lambda^{2}}+\frac{2 \lambda \gamma_{\varepsilon}^{2}}{(1-\lambda)^{2}(1+\lambda)}\right] \\
= & \frac{\sigma_{\varepsilon}^{2}}{1-\lambda^{2}}+\frac{2 \lambda \gamma_{\varepsilon}^{2}}{(1-\lambda)^{2}(1+\lambda)}
\end{aligned}
$$

as $T \rightarrow \infty$ because $\bar{x}_{i}=\frac{1}{T} \sum_{t=1}^{T} x_{i t} \stackrel{p}{\rightarrow} E\left(x_{i t}\right)=0$. Hence,

$$
\frac{1}{n T} \sum_{i=1}^{n} \sum_{t=1}^{T}\left(x_{i t}-\bar{x}_{i}\right)^{2} \stackrel{p}{\rightarrow} \frac{\sigma_{\varepsilon}^{2}}{1-\lambda^{2}}+\frac{2 \lambda \gamma_{\varepsilon}^{2}}{(1-\lambda)^{2}(1+\lambda)}
$$

holds for all $n$ and hence it holds for a large $n$ as well. This proves (1).

Next we consider (2). Note for a fixed $n$

$$
\begin{aligned}
& \frac{1}{n T^{2}} \sum_{i=1}^{n} \sum_{t=1}^{T}\left(x_{i t}-\bar{x}_{i}\right)^{2} \\
= & \frac{1}{n} \sum_{i=1}^{n}\left[\frac{1}{T^{2}} \sum_{t=1}^{T}\left(x_{i t}-\bar{x}_{i}\right)^{2}\right] \\
\Rightarrow & \frac{1}{n} \sum_{i=1}^{n}\left(\varpi_{\varepsilon}^{2} \int \tilde{W}_{i}^{2}\right)
\end{aligned}
$$

as $T \rightarrow \infty$. As $n \rightarrow \infty$,

$$
\frac{1}{n} \sum_{i=1}^{n}\left(\varpi_{\varepsilon}^{2} \int \tilde{W}_{i}^{2}\right) \stackrel{p}{\rightarrow} \frac{\varpi_{\varepsilon}^{2}}{6}
$$


by a LLN since

$$
E\left(\int \tilde{W}_{i}^{2}\right)=\frac{1}{6}
$$

Hence,

$$
\frac{1}{n T^{2}} \sum_{i=1}^{n} \sum_{t=1}^{T}\left(x_{i t}-\bar{x}_{i}\right)^{2} \stackrel{p}{\rightarrow} \frac{\varpi_{\varepsilon}^{2}}{6}
$$

as $(n, T) \stackrel{\text { seq }}{\rightarrow} \infty$. This proves $(2)$.

Lemma 4 If Assumptions $1-2$ hold, then

1. If $|\rho|<1$ and $|\lambda|<1$,
(a) $\frac{1}{n T} \sum_{i=1}^{n} \sum_{t=1}^{T}\left(x_{i t}-\bar{x}_{i}\right) u_{i t} \stackrel{p}{\rightarrow} \lim \frac{1}{n T} \sum_{i=1}^{n} \sum_{t=1}^{T} E\left(x_{i t} \nu_{i t}\right)$
(b) $\frac{1}{\sqrt{n T}} \sum_{i=1}^{n} \sum_{t=1}^{T}\left(x_{i t}-\bar{x}_{i}\right) u_{i t}-\sqrt{n T} \lim \frac{1}{n T} \sum_{i=1}^{n} \sum_{t=1}^{T} E\left(x_{i t} \nu_{i t}\right)$
$\Rightarrow N\left(0, \frac{\psi_{00}+\sum_{r=1}^{\infty} \lambda^{2 r} \psi_{0 r}+\sum_{r=1}^{\infty} \rho^{2 r} \psi_{r 0}}{(1-\rho \lambda)^{2}}\right)$
where $\psi_{0 r}=E\left(\varepsilon_{t-r}^{2} e_{t}^{2}\right), \psi_{r 0}=E\left(\varepsilon_{t}^{2} e_{t-r}^{2}\right)$, and $\psi_{00}=E\left(\varepsilon_{t}^{2} e_{t}^{2}\right)$.

2. If $\rho=1$ and $|\lambda|<1$,
(a) $\frac{1}{n T} \sum_{i=1}^{n} \sum_{t=1}^{T}\left(x_{i t}-\bar{x}_{i}\right) u_{i t} \stackrel{p}{\rightarrow} \frac{-\frac{1}{2} \varpi_{e \varepsilon}+\delta_{e \varepsilon}}{1-\lambda}$
(b) $\frac{1}{\sqrt{n} T} \sum_{i=1}^{n} \sum_{t=1}^{T}\left(x_{i t}-\bar{x}_{i}\right) \nu_{i t}-\sqrt{n}\left[\frac{\left(\frac{1}{n} \sum_{i=1}^{n} \frac{1}{T} \sum_{t=1}^{T}\left(\nu_{i t}-\bar{\nu}_{i}\right) e_{i t}\right) \frac{\varpi_{e \varepsilon}}{\varpi_{e}^{2}}+\delta_{e \varepsilon}}{1-\lambda}\right] \Rightarrow$ $N\left(0, \frac{\varpi_{\varepsilon, e} \varpi_{e}^{2}}{6(1-\lambda)^{2}}\right)$.

3. If $|\rho|<1$ and $\lambda=1$,
(a) $\frac{1}{T} \sum_{t=1}^{T}\left(x_{i t}-\bar{x}_{i}\right) u_{i t} \Rightarrow \frac{1}{1-\rho}\left[\varpi_{\varepsilon} \varpi_{e . \varepsilon}^{1 / 2}\left(\int \widetilde{W}_{i} d V_{i}\right)+\varpi_{\varepsilon e}\left(\int \widetilde{W}_{i} d W_{i}^{\prime}\right)+\delta_{\varepsilon e}\right]$,
(b) $\frac{1}{n T} \sum_{i=1}^{n} \sum_{t=1}^{T}\left(x_{i t}-\bar{x}_{i}\right) u_{i t} \stackrel{p}{\rightarrow} \frac{-\frac{1}{2} \varpi_{\varepsilon e}+\delta_{\varepsilon e}}{1-\rho}$,
(c) $\frac{1}{\sqrt{n} T} \sum_{i=1}^{n} \sum_{t=1}^{T}\left(x_{i t}-\bar{x}_{i}\right) \nu_{i t}-\sqrt{n}\left[\frac{\left(\frac{1}{n} \sum_{i=1}^{n} \frac{1}{T} \sum_{t=1}^{T}\left(x_{i t}-\bar{x}_{i}\right) \varepsilon_{i t}\right) \frac{\varpi_{\varepsilon e}}{\omega_{\varepsilon}^{2}}+\delta_{\varepsilon e}}{1-\rho}\right] \Rightarrow$ $N\left(0, \frac{\varpi_{e . \varepsilon} \varpi_{\varepsilon}^{2}}{6(1-\rho)^{2}}\right)$.

4. If $\rho=1$ and $\lambda=1$,
(a) $\frac{1}{T^{2}} \sum_{t=1}^{T}\left(x_{i t}-\bar{x}_{i}\right) u_{i t} \Rightarrow \varpi_{\varepsilon} \varpi_{e . \varepsilon}^{1 / 2}\left(\int \widetilde{W}_{i} \tilde{V}_{i}\right)+\varpi_{\varepsilon e}\left(\int \widetilde{W}_{i}^{2}\right)+\delta_{\varepsilon e}$,
(b) $\frac{1}{n} \sum_{i=1}^{n}\left[\frac{1}{T^{2}} \sum_{t=1}^{T}\left(x_{i t}-\bar{x}_{i}\right) u_{i t}\right] \stackrel{p}{\rightarrow} \frac{\varpi_{\varepsilon e}}{6}+\delta_{\varepsilon e}$, 

(c) $\frac{1}{\sqrt{n}} \sum_{i=1}^{n}\left[\frac{1}{T^{2}} \sum_{t=1}^{T}\left(x_{i t}-\bar{x}_{i}\right) u_{i t}\right]-\sqrt{n}\left[\left(\frac{1}{n} \sum_{i=1}^{n} \frac{1}{T^{2}} \sum_{t=1}^{T}\left(x_{i t}-\bar{x}_{i}\right)^{2}\right) \frac{\varpi_{\varepsilon e}}{\varpi_{\varepsilon}^{2}}+\delta_{\varepsilon e}\right] \Rightarrow$ $N\left(0, \frac{\varpi_{e . \varepsilon} \varpi_{\varepsilon}^{2}}{90}\right)$.

\section{Proof.}

Consider (1). For part (a), note that

$$
\begin{aligned}
& \frac{1}{n T} \sum_{i=1}^{n} \sum_{t=1}^{T}\left(x_{i t}-\bar{x}_{i}\right) \nu_{i t} \\
= & \frac{1}{n} \sum_{i=1}^{n}\left[\frac{1}{T} \sum_{t=1}^{T} x_{i t} \nu_{i t}\right]-\frac{1}{T} \frac{1}{n} \sum_{i=1}^{n}\left[\left(\frac{1}{\sqrt{T}} \sum_{t=1}^{T} x_{i t}\right)\left(\frac{1}{\sqrt{T}} \sum_{t=1}^{T} \nu_{i t}\right)\right]
\end{aligned}
$$

Because

$$
\frac{1}{n} \sum_{i=1}^{n}\left[\frac{1}{T} \sum_{t=1}^{T} x_{i t} \nu_{i t}\right]=\lim \frac{1}{n T} \sum_{i=1}^{n} \sum_{t=1}^{T} E\left(x_{i t} \nu_{i t}\right)+o_{p}(1)
$$

as $(n, T) \stackrel{\text { seq }}{\rightarrow} \infty$. Also it is easy to see that

$$
\frac{1}{T} \frac{1}{n} \sum_{i=1}^{n}\left[\left(\frac{1}{\sqrt{T}} \sum_{t=1}^{T} x_{i t}\right)\left(\frac{1}{\sqrt{T}} \sum_{t=1}^{T} \nu_{i t}\right)\right]=o_{p}(1) .
$$

Hence, we have

$$
\frac{1}{n T} \sum_{i=1}^{n} \sum_{t=1}^{T}\left(x_{i t}-\bar{x}_{i}\right) \nu_{i t}=\lim \frac{1}{n T} \sum_{i=1}^{n} \sum_{t=1}^{T} E\left(x_{i t} \nu_{i t}\right)+o_{p}(1) .
$$

This proves (a).

For part (b), for a fixed $n$,

$$
\begin{aligned}
& \frac{1}{\sqrt{n T}} \sum_{i=1}^{n} \sum_{t=1}^{T}\left(x_{i t}-\bar{x}_{i}\right) \nu_{i t} \\
= & \frac{1}{\sqrt{n}} \sum_{i=1}^{n}\left(\frac{1}{\sqrt{T}} \sum_{t=1}^{T} x_{i t} \nu_{i t}\right)-\frac{1}{\sqrt{T}} \frac{1}{\sqrt{n}} \sum_{i=1}^{n}\left[\left(\frac{1}{\sqrt{T}} \sum_{t=1}^{T} x_{i t}\right)\left(\frac{1}{\sqrt{T}} \sum_{t=1}^{T} \nu_{i t}\right)\right]
\end{aligned}
$$

Because

$$
\begin{aligned}
& \frac{1}{\sqrt{n}} \sum_{i=1}^{n}\left(\frac{1}{\sqrt{T}} \sum_{t=1}^{T} x_{i t} \nu_{i t}\right)-\sqrt{n T} \lim \frac{1}{n T} \sum_{i=1}^{n} \sum_{t=1}^{T} E\left(x_{i t} \nu_{i t}\right) \\
\Rightarrow & N\left(0, \frac{\psi_{00}+\sum_{r=1}^{\infty} \lambda^{2 r} \psi_{0 r}+\sum_{r=1}^{\infty} \rho^{2 r} \psi_{r 0}}{(1-\rho \lambda)^{2}}\right)
\end{aligned}
$$


as $(n, T) \stackrel{\text { seq }}{\rightarrow} \infty$, where $\psi_{0 r}=E\left(\varepsilon_{t-r}^{2} e_{t}^{2}\right), \psi_{r 0}=E\left(\varepsilon_{t}^{2} e_{t-r}^{2}\right), \psi_{00}=E\left(\varepsilon_{t}^{2} e_{t}^{2}\right)$.

Also it is easy to see that

$$
\frac{1}{\sqrt{T}} \frac{1}{\sqrt{n}} \sum_{i=1}^{n}\left[\left(\frac{1}{\sqrt{T}} \sum_{t=1}^{T} x_{i t}\right)\left(\frac{1}{\sqrt{T}} \sum_{t=1}^{T} \nu_{i t}\right)\right]=o_{p}(1)
$$

Hence, we have

$$
\begin{aligned}
& \frac{1}{\sqrt{n T}} \sum_{i=1}^{n} \sum_{t=1}^{T}\left(x_{i t}-\bar{x}_{i}\right) \nu_{i t}-\sqrt{n T} \lim \frac{1}{n T} \sum_{i=1}^{n} \sum_{t=1}^{T} E\left(x_{i t} \nu_{i t}\right) \\
= & {\left[\frac{1}{\sqrt{n}} \sum_{i=1}^{n}\left(\frac{1}{\sqrt{T}} \sum_{t=1}^{T} x_{i t} \nu_{i t}\right)-\sqrt{n T} \lim \frac{1}{n T} \sum_{i=1}^{n} \sum_{t=1}^{T} E\left(x_{i t} \nu_{i t}\right)\right] } \\
& -\frac{1}{\sqrt{T}} \frac{1}{\sqrt{n}} \sum_{i=1}^{n}\left[\left(\frac{1}{\sqrt{T}} \sum_{t=1}^{T} x_{i t}\right)\left(\frac{1}{\sqrt{T}} \sum_{t=1}^{T} \nu_{i t}\right)\right] \\
\Rightarrow & N\left(0, \frac{\psi_{00}+\sum_{r=1}^{\infty} \lambda^{2 r} \psi_{0 r}+\sum_{r=1}^{\infty} \rho^{2 r} \psi_{r 0}}{(1-\rho \lambda)^{2}}\right) .
\end{aligned}
$$

Consider (2). For part (a), for a fixed $n$, note that

$$
\begin{aligned}
& \frac{1}{n T} \sum_{i=1}^{n} \sum_{t=1}^{T}\left(x_{i t}-\bar{x}_{i}\right) \nu_{i t} \\
= & \frac{1}{n} \sum_{i=1}^{n}\left[\frac{1}{T} \sum_{t=1}^{T} x_{i t}\left(\nu_{i t}-\bar{\nu}_{i}\right)\right] \\
\Rightarrow & \frac{1}{n} \sum_{i=1}^{n}\left[\frac{\varpi_{e} \varpi_{\varepsilon \cdot e}^{1 / 2}\left(\int \widetilde{V}_{i} d W_{i}\right)+\varpi_{e \varepsilon}\left(\int \widetilde{V}_{i} d V_{i}\right)+\delta_{e \varepsilon}}{1-\lambda}\right]
\end{aligned}
$$

as $T \rightarrow \infty$, by a CLT because

$$
\begin{aligned}
& \frac{1}{n} \sum_{i=1}^{n}\left[\frac{\varpi_{e} \varpi_{\varepsilon \cdot e}^{1 / 2}\left(\int \widetilde{V}_{i} d W_{i}\right)+\varpi_{e \varepsilon}\left(\int \widetilde{V}_{i} d V_{i}\right)+\delta_{e \varepsilon}}{1-\lambda}\right] \\
& \stackrel{p}{\rightarrow} \frac{-\frac{1}{2} \varpi_{e \varepsilon}+\delta_{e \varepsilon}}{1-\lambda}
\end{aligned}
$$

as $(n, T) \stackrel{\text { seq }}{\longrightarrow} \infty$. Hence, we have

$$
\frac{1}{n T} \sum_{i=1}^{n} \sum_{t=1}^{T}\left(x_{i t}-\bar{x}_{i}\right) \nu_{i t} \stackrel{p}{\rightarrow} \frac{-\frac{1}{2} \varpi_{e \varepsilon}+\delta_{e \varepsilon}}{1-\lambda}
$$

as $(n, T) \stackrel{\text { seq }}{\longrightarrow} \infty$. 
For part (b), for a fixed $n$,

$$
\begin{aligned}
& \frac{1}{\sqrt{n} T} \sum_{i=1}^{n} \sum_{t=1}^{T}\left(x_{i t}-\bar{x}_{i}\right) \nu_{i t} \\
= & \frac{1}{\sqrt{n}} \sum_{i=1}^{n}\left[\frac{1}{T} \sum_{t=1}^{T} x_{i t}\left(\nu_{i t}-\bar{\nu}_{i}\right)\right] \\
\Rightarrow & \frac{1}{\sqrt{n}} \sum_{i=1}^{n}\left[\frac{\varpi_{e} \varpi_{\varepsilon \cdot e}^{1 / 2}\left(\int \widetilde{V}_{i} d W_{i}\right)+\varpi_{e \varepsilon}\left(\int \widetilde{V}_{i} d V_{i}\right)+\delta_{e \varepsilon}}{1-\lambda}\right]
\end{aligned}
$$

as $T \rightarrow \infty$, by a CLT. Hence, we have

$$
\begin{aligned}
& \frac{1}{\sqrt{n} T} \sum_{i=1}^{n} \sum_{t=1}^{T}\left(x_{i t}-\bar{x}_{i}\right) \nu_{i t}-\sqrt{n}\left[\frac{\left(\frac{1}{n} \sum_{i=1}^{n} \frac{1}{T} \sum_{t=1}^{T}\left(\nu_{i t}-\bar{\nu}_{i}\right) e_{i t}\right) \frac{\varpi_{e \varepsilon}}{\varpi_{e}^{2}}+\delta_{e \varepsilon}}{1-\lambda}\right] \\
\Rightarrow & \frac{1}{\sqrt{n}} \sum_{i=1}^{n}\left[\frac{\varpi_{e} \varpi_{\varepsilon . e}^{1 / 2}\left(\int \widetilde{V}_{i} d W_{i}\right)+\varpi_{e \varepsilon}\left(\int \widetilde{V}_{i} d V_{i}\right)+\delta_{e \varepsilon}}{1-\lambda}\right]-\frac{1}{\sqrt{n}} \sum_{i=1}^{n}\left[\frac{\varpi_{e \varepsilon}\left(\int \widetilde{V}_{i} d V_{i}\right)+\delta_{e \varepsilon}}{1-\lambda}\right] \\
= & \frac{1}{\sqrt{n}} \sum_{i=1}^{n}\left[\frac{\varpi_{e} \varpi_{\varepsilon \cdot e}^{1 / 2}\left(\int \widetilde{V}_{i} d W_{i}\right)}{1-\lambda}\right] \\
\Rightarrow & N\left(0, \frac{\varpi_{\varepsilon . e} \varpi_{e}^{2}}{6(1-\lambda)^{2}}\right) .
\end{aligned}
$$

as $(n, T) \stackrel{\text { seq }}{\rightarrow} \infty$.

Consider (3). For part (a), note that for a fixed $n$,

$$
\begin{aligned}
& \frac{1}{n T} \sum_{i=1}^{n} \sum_{t=1}^{T}\left(x_{i t}-\bar{x}_{i}\right) \nu_{i t} \\
= & \frac{1}{n} \sum_{i=1}^{n}\left[\frac{1}{T} \sum_{t=1}^{T}\left(x_{i t}-\bar{x}_{i}\right) \nu_{i t}\right] \\
\Rightarrow & \frac{1}{n} \sum_{i=1}^{n}\left[\frac{\varpi_{\varepsilon} \varpi_{e . \varepsilon}^{1 / 2}\left(\int \widetilde{W}_{i} d V_{i}\right)+\varpi_{\varepsilon e}\left(\int \widetilde{W}_{i} d W_{i}\right)+\delta_{\varepsilon e}}{1-\rho}\right]
\end{aligned}
$$

as $T \rightarrow \infty$, by a central limit theorem. And

$$
\begin{aligned}
& \frac{1}{n} \sum_{i=1}^{n}\left[\frac{\varpi_{\varepsilon} \varpi_{e . \varepsilon}^{1 / 2}\left(\int \widetilde{W}_{i} d V_{i}\right)+\varpi_{\varepsilon e}\left(\int \widetilde{W}_{i} d W_{i}\right)+\delta_{\varepsilon e}}{1-\rho}\right] \\
& \stackrel{p}{\rightarrow} \frac{-\frac{1}{2} \varpi_{\varepsilon e}+\delta_{\varepsilon e}}{1-\rho},
\end{aligned}
$$


as $n \rightarrow \infty$. Hence, we have

$$
\frac{1}{n T} \sum_{i=1}^{n} \sum_{t=1}^{T}\left(x_{i t}-\bar{x}_{i}\right) \nu_{i t} \stackrel{p}{\rightarrow} \frac{-\frac{1}{2} \varpi_{\varepsilon e}+\delta_{\varepsilon e}}{1-\rho}
$$

as $(n, T) \stackrel{\text { seq }}{\rightarrow} \infty$.

For part (b), for a fixed $n$,

$$
\begin{aligned}
& \frac{1}{\sqrt{n} T} \sum_{i=1}^{n} \sum_{t=1}^{T}\left(x_{i t}-\bar{x}_{i}\right) \nu_{i t} \\
= & \frac{1}{\sqrt{n}} \sum_{i=1}^{n}\left[\frac{1}{T} \sum_{t=1}^{T}\left(x_{i t}-\bar{x}_{i}\right) \nu_{i t}\right] \\
\Rightarrow & \frac{1}{\sqrt{n}} \sum_{i=1}^{n}\left[\frac{\varpi_{\varepsilon} \varpi_{e . \varepsilon}^{1 / 2}\left(\int \widetilde{W}_{i} d V_{i}\right)+\varpi_{\varepsilon e}\left(\int \widetilde{W}_{i} d W_{i}\right)+\delta_{\varepsilon e}}{1-\rho}\right],
\end{aligned}
$$

as $T \rightarrow \infty$, by a central limit theorem. Hence, we have

$$
\begin{aligned}
& \frac{1}{\sqrt{n} T} \sum_{i=1}^{n} \sum_{t=1}^{T}\left(x_{i t}-\bar{x}_{i}\right) \nu_{i t}-\sqrt{n}\left[\frac{\left(\frac{1}{n} \sum_{i=1}^{n} \frac{1}{T} \sum_{t=1}^{T}\left(x_{i t}-\bar{x}_{i}\right) \varepsilon_{i t}\right) \frac{\varpi_{\varepsilon e}}{\varpi_{\varepsilon}}+\delta_{\varepsilon e}}{1-\rho}\right] \\
& \Rightarrow \frac{1}{\sqrt{n}} \sum_{i=1}^{n}\left[\frac{\varpi_{\varepsilon} \varpi_{e . \varepsilon}^{1 / 2}\left(\int \widetilde{W}_{i} d V_{i}\right)+\varpi_{\varepsilon e}\left(\int \widetilde{W}_{i} d W_{i}\right)+\delta_{\varepsilon e}}{1-\rho}\right] \\
&-\frac{1}{\sqrt{n}} \sum_{i=1}^{n}\left[\frac{\varpi_{\varepsilon e}\left(\int \widetilde{W}_{i} d W_{i}\right)+\delta_{\varepsilon e}}{1-\rho}\right] \\
&= \frac{1}{\sqrt{n}} \sum_{i=1}^{n}\left[\frac{\varpi_{\varepsilon} \varpi_{e . \varepsilon}^{1 / 2}\left(\int \widetilde{W}_{i} d V_{i}\right)}{1-\rho}\right] \\
& \Rightarrow \quad N\left(0, \frac{\varpi_{e . \varepsilon} \varpi_{\varepsilon}^{2}}{6(1-\rho)^{2}}\right) . \\
& \text { as }(n, T) \stackrel{\text { seq }}{\rightarrow} \infty . \\
& \text { If } \rho=1 \text { and } \lambda=1,
\end{aligned}
$$


Consider (4). For part (a), note that

$$
\begin{aligned}
& \frac{1}{n T^{2}} \sum_{i=1}^{n} \sum_{t=1}^{T}\left(x_{i t}-\bar{x}_{i}\right) \nu_{i t} \\
= & \frac{1}{n} \sum_{i=1}^{n}\left[\frac{1}{T^{2}} \sum_{t=1}^{T}\left(x_{i t}-\bar{x}_{i}\right) \nu_{i t}\right] \\
\Rightarrow & \frac{1}{n} \sum_{i=1}^{n}\left[\varpi_{\varepsilon} \varpi_{e . \varepsilon}^{1 / 2}\left(\int \widetilde{W}_{i} \tilde{V}_{i}\right)+\left(\int \widetilde{W}_{i}^{2}\right) \varpi_{\varepsilon e}+\delta_{\varepsilon e}\right]
\end{aligned}
$$

as $T \rightarrow \infty$, by a central limit theorem. And

$$
\begin{aligned}
& \frac{1}{n} \sum_{i=1}^{n}\left[\varpi_{\varepsilon} \varpi_{e . \varepsilon}^{1 / 2}\left(\int \widetilde{W}_{i} \tilde{V}_{i}\right)+\left(\int \widetilde{W}_{i}^{2}\right) \varpi_{\varepsilon e}+\delta_{\varepsilon e}\right] \\
& \stackrel{p}{\rightarrow} \frac{\varpi_{\varepsilon e}}{6}+\delta_{\varepsilon e}
\end{aligned}
$$

as $n \rightarrow \infty$. Hence, we have

$$
\frac{1}{n T^{2}} \sum_{i=1}^{n} \sum_{t=1}^{T}\left(x_{i t}-\bar{x}_{i}\right) \nu_{i t} \stackrel{p}{\rightarrow} \frac{\varpi_{\varepsilon e}}{6}+\delta_{\varepsilon e}
$$

as $(n, T) \stackrel{\text { seq }}{\rightarrow} \infty$.

For part (b), for a fixed $n$,

$$
\begin{aligned}
& \frac{1}{\sqrt{n} T^{2}} \sum_{i=1}^{n} \sum_{t=1}^{T}\left(x_{i t}-\bar{x}_{i}\right) \nu_{i t} \\
= & \frac{1}{\sqrt{n}} \sum_{i=1}^{n}\left[\frac{1}{T^{2}} \sum_{t=1}^{T}\left(x_{i t}-\bar{x}_{i}\right) \nu_{i t}\right] \\
\Rightarrow & \frac{1}{\sqrt{n}} \sum_{i=1}^{n}\left[\varpi_{\varepsilon} \varpi_{e . \varepsilon}^{1 / 2}\left(\int \widetilde{W}_{i} \tilde{V}_{i}\right)+\left(\int \widetilde{W}_{i}^{2}\right) \varpi_{\varepsilon e}+\delta_{\varepsilon e}\right],
\end{aligned}
$$

as $T \rightarrow \infty$, by a central limit theorem. Hence, we have

$$
\begin{aligned}
& \frac{1}{\sqrt{n} T^{2}} \sum_{i=1}^{n} \sum_{t=1}^{T}\left(x_{i t}-\bar{x}_{i}\right) \nu_{i t}-\sqrt{n}\left[\left(\frac{1}{n} \sum_{i=1}^{n} \frac{1}{T^{2}} \sum_{t=1}^{T}\left(x_{i t}-\bar{x}_{i}\right)^{2}\right) \frac{\varpi_{\varepsilon e}}{\varpi_{\varepsilon}^{2}}+\delta_{\varepsilon e}\right] \\
\Rightarrow & \frac{1}{\sqrt{n}} \sum_{i=1}^{n}\left[\varpi_{\varepsilon} \varpi_{e . \varepsilon}^{1 / 2}\left(\int \widetilde{W}_{i} \tilde{V}_{i}\right)+\left(\int \widetilde{W}_{i}^{2}\right) \varpi_{\varepsilon e}+\delta_{\varepsilon e}\right]-\frac{1}{\sqrt{n}} \sum_{i=1}^{n}\left[\left(\int \widetilde{W}_{i}^{2}\right) \varpi_{\varepsilon e}+\delta_{\varepsilon e}\right] \\
= & \frac{1}{\sqrt{n}} \sum_{i=1}^{n}\left[\varpi_{\varepsilon} \varpi_{e . \varepsilon}^{1 / 2}\left(\int \widetilde{W}_{i} \tilde{V}_{i}\right)\right] \\
\Rightarrow & N\left(0, \frac{\varpi_{e . \varepsilon} \varpi_{\varepsilon}^{2}}{90}\right) .
\end{aligned}
$$


as $(n, T) \stackrel{\text { seq }}{\rightarrow} \infty$

\section{Proof of Theorem 2}

Proof. Te proof of Theorem 2 is straightforward with above lemmas.

\section{E Proof of Theorem 3}

The following lemmas will be used to prove Theorem 3 .

Lemma 5 If Assumptions $1-2$ hold, then

1. If $|\lambda|<1, \frac{1}{n T} \sum_{i=1}^{n} \sum_{t=1}^{T}\left(x_{i t}-x_{i t-1}\right)^{2} \stackrel{p}{\rightarrow} \frac{2 \sigma_{\varepsilon}^{2}}{1+\lambda}+\frac{2\left(-2 \lambda^{2}+2 \lambda-1\right) \gamma_{\varepsilon}^{2}}{1-\lambda}$,

2. If $\lambda=1, \frac{1}{n T} \sum_{i=1}^{n} \sum_{t=1}^{T}\left(x_{i t}-x_{i t-1}\right)^{2} \stackrel{p}{\rightarrow} \sigma_{\varepsilon}^{2}$.

Proof. Consider (1). If $|\lambda|<1$, for a fixed $n$, it is clear to see that

$$
\begin{aligned}
& \frac{1}{n T} \sum_{i=1}^{n} \sum_{t=1}^{T}\left(x_{i t}-x_{i t-1}\right)^{2} \\
= & \frac{1}{n} \sum_{i=1}^{n}\left[\frac{1}{T} \sum_{t=1}^{T}\left(x_{i t}-x_{i t-1}\right)^{2}\right] \\
= & \frac{1}{n} \sum_{i=1}^{n}\left[\frac{1}{T} \sum_{t=1}^{T} x_{i t}^{2}+\frac{1}{T} \sum_{t=1}^{T} x_{i t-1}^{2}-\frac{2}{T} \sum_{t=1}^{T} x_{i t} x_{i t-1}\right] \\
& \frac{p}{\rightarrow} \frac{1}{n} \sum_{i=1}^{n}\left[2\left(\frac{\sigma_{\varepsilon}^{2}}{1-\lambda^{2}}+\frac{2 \lambda \gamma_{\varepsilon}^{2}}{1-\lambda}\right)-2\left[\lambda\left(\frac{\sigma_{\varepsilon}^{2}}{1-\lambda^{2}}+\frac{2 \lambda \gamma_{\varepsilon}^{2}}{1-\lambda}\right)+\frac{\gamma_{\varepsilon}^{2}}{1-\lambda}\right]\right] \\
= & \frac{1}{n} \sum_{i=1}^{n}\left[\frac{2 \sigma_{\varepsilon}^{2}}{1+\lambda}+\frac{2\left(-2 \lambda^{2}+2 \lambda-1\right) \gamma_{\varepsilon}^{2}}{1-\lambda}\right] \\
& \frac{p}{\rightarrow} \frac{2 \sigma_{\varepsilon}^{2}}{1+\lambda}+\frac{2\left(-2 \lambda^{2}+2 \lambda-1\right) \gamma_{\varepsilon}^{2}}{1-\lambda}
\end{aligned}
$$

as $T \rightarrow \infty$ because $\frac{1}{T} \sum_{t=1}^{T} x_{i t}^{2} \stackrel{p}{\rightarrow} E\left(x_{i t}^{2}\right)=\frac{\sigma_{\varepsilon}^{2}}{1-\lambda^{2}}+\frac{2 \lambda \gamma_{\varepsilon}^{2}}{1-\lambda}$ and $\frac{1}{T} \sum_{t=1}^{T} x_{i t} x_{i t-1} \stackrel{p}{\rightarrow}$ $E\left(x_{i t} x_{i t-1}\right)=\lambda\left(\frac{\sigma_{\varepsilon}^{2}}{1-\lambda^{2}}+\frac{2 \lambda \gamma_{\varepsilon}^{2}}{1-\lambda}\right)+\frac{\gamma_{\varepsilon}^{2}}{1-\lambda}$. Hence,

$$
\frac{1}{n T} \sum_{i=1}^{n} \sum_{t=1}^{T}\left(x_{i t}-\bar{x}_{i}\right)^{2} \stackrel{p}{\rightarrow} \frac{2 \sigma_{\varepsilon}^{2}}{1+\lambda}+\frac{2\left(-2 \lambda^{2}+2 \lambda-1\right) \gamma_{\varepsilon}^{2}}{1-\lambda}
$$

holds for all $n$ and hence it holds for a large $n$ as well. This proves (1). 
Next we consider (2). If $\lambda=1$,

$$
\begin{aligned}
& \frac{1}{n T} \sum_{i=1}^{n} \sum_{t=1}^{T}\left(x_{i t}-x_{i t-1}\right)^{2} \\
= & \frac{1}{n} \sum_{i=1}^{n}\left[\frac{1}{T} \sum_{t=1}^{T}\left(x_{i t}-x_{i t-1}\right)^{2}\right] \\
= & \frac{1}{n} \sum_{i=1}^{n}\left[\frac{1}{T} \sum_{t=1}^{T} \varepsilon_{i t}^{2}\right] \stackrel{p}{\rightarrow} \sigma_{\varepsilon}^{2}
\end{aligned}
$$

as $(n, T) \stackrel{\text { seq }}{\rightarrow} \infty$. This proves $(2)$.

Lemma 6 If Assumptions $1-2$ hold, then

1. If $|\rho|<1$ and $|\lambda|<1$,

(a) $\frac{1}{n} \sum_{i=1}^{n}\left[\frac{1}{T} \sum_{t=1}^{T}\left(x_{i t}-x_{i t-1}\right)\left(\nu_{i t}-\nu_{i t-1}\right)\right] \stackrel{p}{\rightarrow} \lim \frac{1}{n T} \sum_{i=1}^{n} \sum_{t=1}^{T} E\left(x_{i t}-x_{i t-1}\right)\left(\nu_{i t}-\nu_{i t-1}\right)$

(b) $\frac{1}{\sqrt{n}} \sum_{i=1}^{n}\left[\frac{1}{\sqrt{T}} \sum_{t=1}^{T}\left(x_{i t}-x_{i t-1}\right)\left(\nu_{i t}-\nu_{i t-1}\right)\right]-\sqrt{n T} \lim \frac{1}{n T} \sum_{i=1}^{n} \sum_{t=1}^{T} E\left(x_{i t}-x_{i t-1}\right)\left(\nu_{i t}-\nu_{i t-1}\right.$ $\Rightarrow N\left(0, \frac{(2-\lambda-\rho)^{2} \psi_{00}+\sum_{r=1}^{\infty}\left(-\rho^{r-1}+2 \rho^{r}-\rho^{r+1}\right)^{2} \psi_{0 r}+\sum_{r=1}^{\infty}\left(-\lambda^{r-1}+2 \lambda^{r}-\lambda^{r+1}\right)^{2} \psi_{r 0}}{(1-\rho \lambda)^{2}}\right)$

where $\psi_{0 r}=E\left(\varepsilon_{t-r}^{2} e_{t}^{2}\right), \psi_{r 0}=E\left(\varepsilon_{t}^{2} e_{t-r}^{2}\right), \psi_{00}=E\left(\varepsilon_{t}^{2} e_{t}^{2}\right)$.

2. If $\rho=1$ and $|\lambda|<1$,

(a) $\frac{1}{n} \sum_{i=1}^{n}\left[\frac{1}{T} \sum_{t=1}^{T}\left(x_{i t}-x_{i t-1}\right)\left(\nu_{i t}-\nu_{i t-1}\right)\right] \stackrel{p}{\rightarrow} \lim \frac{1}{n T} \sum_{i=1}^{n} \sum_{t=1}^{T} E\left(\varepsilon_{i t}+(\lambda-1) x_{i t-1}\right) e_{i t}$

(b) $\frac{1}{\sqrt{n}} \sum_{i=1}^{n}\left[\frac{1}{T} \sum_{t=1}^{T}\left(x_{i t}-x_{i t-1}\right)\left(\nu_{i t}-\nu_{i t-1}\right)\right]-\sqrt{n} \lim \frac{1}{n T} \sum_{i=1}^{n} \sum_{t=1}^{T} E\left(\varepsilon_{i t}+(\lambda-1) x_{i t-1}\right) e_{i t} \Rightarrow$ $N\left(0, \frac{2 \psi_{00}}{1+\lambda}\right)$, where $\psi_{00}=E\left(\varepsilon_{t}^{2} e_{t}^{2}\right)$

3. If $|\rho|<1$ and $\lambda=1$,

(a) $\frac{1}{n} \sum_{i=1}^{n}\left[\frac{1}{T} \sum_{t=1}^{T}\left(x_{i t}-x_{i t-1}\right)\left(\nu_{i t}-\nu_{i t-1}\right)\right] \stackrel{p}{\rightarrow} \lim \frac{1}{n T} \sum_{i=1}^{n} \sum_{t=1}^{T} \varepsilon_{i t}\left(\nu_{i t}-\nu_{i t-1}\right)$

(b) $\frac{1}{\sqrt{n}} \sum_{i=1}^{n}\left[\frac{1}{T} \sum_{t=1}^{T}\left(x_{i t}-x_{i t-1}\right)\left(\nu_{i t}-\nu_{i t-1}\right)\right]-\sqrt{n} \lim \frac{1}{n T} \sum_{i=1}^{n} \sum_{t=1}^{T} \varepsilon_{i t}\left(\nu_{i t}-\nu_{i t-1}\right) \Rightarrow$ $N\left(0, \frac{2 \psi_{00}}{1+\rho}\right)$, where $\psi_{00}=E\left(\varepsilon_{t}^{2} e_{t}^{2}\right)$.

4. If $\rho=1$ and $\lambda=1$,

(a) $\frac{1}{n} \sum_{i=1}^{n}\left[\frac{1}{T} \sum_{t=1}^{T}\left(x_{i t}-x_{i t-1}\right)\left(\nu_{i t}-\nu_{i t-1}\right)\right] \stackrel{p}{\rightarrow} \sigma_{\varepsilon e}$ 
(b) $\frac{1}{\sqrt{n}} \sum_{i=1}^{n}\left[\frac{1}{\sqrt{T}} \sum_{t=1}^{T}\left(x_{i t}-x_{i t-1}\right)\left(\nu_{i t}-\nu_{i t-1}\right)\right]-\sqrt{n T} \sigma_{\varepsilon e} \Rightarrow N\left(0, \varpi_{e}^{2} \varpi_{\varepsilon}^{2}\right)$.

Proof. Consider (1). For part (a), it is easy to see that

$$
\begin{aligned}
& \frac{1}{n T} \sum_{i=1}^{n} \sum_{t=1}^{T}\left(x_{i t}-x_{i t-1}\right)\left(\nu_{i t}-\nu_{i t-1}\right) \\
& \stackrel{p}{\rightarrow} \lim \frac{1}{n T} \sum_{i=1}^{n} \sum_{t=1}^{T} E\left(x_{i t}-x_{i t-1}\right)\left(\nu_{i t}-\nu_{i t-1}\right)
\end{aligned}
$$

as $(n, T) \stackrel{\text { seq }}{\longrightarrow} \infty$.

For part (b), note that

$$
\begin{aligned}
& \frac{1}{\sqrt{n T}} \sum_{i=1}^{n} \sum_{t=1}^{T}\left(x_{i t}-x_{i t-1}\right)\left(\nu_{i t}-\nu_{i t-1}\right) \\
= & \frac{1}{\sqrt{n}} \sum_{i=1}^{n}\left[\frac{1}{\sqrt{T}} \sum_{t=1}^{T}\left(\varepsilon_{i t}+(\lambda-1) x_{i t-1}\right)\left(e_{i t}+(\rho-1) \nu_{i t-1}\right)\right] \\
= & \frac{1}{\sqrt{n}} \sum_{i=1}^{n}\left[\frac{1}{\sqrt{T}} \sum_{t=1}^{T}\left(\varepsilon_{i t}+(\lambda-1) \varepsilon_{i t-1}+\lambda(\lambda-1) \varepsilon_{i t-2}+\lambda^{2}(\lambda-1) \varepsilon_{i t-3}+\cdots\right)\right. \\
& \left.\left(e_{i t}+(\rho-1) e_{i t-1}+\rho(\rho-1) e_{i t-2}+\rho^{2}(\rho-1) e_{i t-3}+\cdots\right)\right]
\end{aligned}
$$

Hence, we have

$$
\begin{aligned}
& \frac{1}{\sqrt{n T}} \sum_{i=1}^{n} \sum_{t=1}^{T}\left(x_{i t}-x_{i t-1}\right)\left(\nu_{i t}-\nu_{i t-1}\right)-\sqrt{n T} \lim \frac{1}{n T} \sum_{i=1}^{n} \sum_{t=1}^{T} E\left(x_{i t}-x_{i t-1}\right)\left(\nu_{i t}-\nu_{i t-1}\right) \\
= & \frac{1}{\sqrt{n}} \sum_{i=1}^{n}\left[\frac{1}{\sqrt{T}} \sum_{t=1}^{T}\left(\varepsilon_{i t}+(\lambda-1) \varepsilon_{i t-1}+\lambda(\lambda-1) \varepsilon_{i t-2}+\lambda^{2}(\lambda-1) \varepsilon_{i t-3}+\cdots\right)\right. \\
& \left.\left(e_{i t}+(\rho-1) e_{i t-1}+\rho(\rho-1) e_{i t-2}+\rho^{2}(\rho-1) e_{i t-3}+\cdots\right)\right] \\
& -\sqrt{n T} \lim \frac{1}{n T} \sum_{i=1}^{n} \sum_{t=1}^{T} E\left(x_{i t}-x_{i t-1}\right)\left(\nu_{i t}-\nu_{i t-1}\right) \\
\Rightarrow & N\left(0, \frac{(2-\lambda-\rho)^{2} \psi_{00}+\sum_{r=1}^{\infty}\left(-\rho^{r-1}+2 \rho^{r}-\rho^{r+1}\right)^{2} \psi_{0 r}+\sum_{r=1}^{\infty}\left(-\lambda^{r-1}+2 \lambda^{r}-\lambda^{r+1}\right)^{2} \psi_{r 0}}{(1-\rho \lambda)^{2}}\right)
\end{aligned}
$$

as $(n, T) \stackrel{\text { seq }}{\rightarrow} \infty$, where $\psi_{0 r}=E\left(\varepsilon_{t-r}^{2} e_{t}^{2}\right), \psi_{r 0}=E\left(\varepsilon_{t}^{2} e_{t-r}^{2}\right), \psi_{00}=E\left(\varepsilon_{t}^{2} e_{t}^{2}\right)$. 
Consider (2). For part (a), it is easy to see that

$$
\begin{aligned}
& \frac{1}{n T} \sum_{i=1}^{n} \sum_{t=1}^{T}\left(x_{i t}-x_{i t-1}\right)\left(\nu_{i t}-\nu_{i t-1}\right) \\
= & \frac{1}{n} \sum_{i=1}^{n}\left[\frac{1}{T} \sum_{t=1}^{T}\left(\varepsilon_{i t}+(\lambda-1) x_{i t-1}\right) e_{i t}\right] \\
& \stackrel{p}{\rightarrow} \lim \frac{1}{n T} \sum_{i=1}^{n} \sum_{t=1}^{T} E\left(\varepsilon_{i t}+(\lambda-1) x_{i t-1}\right) e_{i t}
\end{aligned}
$$

as $(n, T) \stackrel{\text { seq }}{\longrightarrow} \infty$.

For part (b), using Lemma A0 in Choi (1999), we have

$$
\begin{aligned}
& \frac{1}{\sqrt{n} T} \sum_{i=1}^{n} \sum_{t=1}^{T}\left(x_{i t}-x_{i t-1}\right)\left(\nu_{i t}-\nu_{i t-1}\right)-\sqrt{n} \lim \frac{1}{n T} \sum_{i=1}^{n} \sum_{t=1}^{T} E\left(\varepsilon_{i t}+(\lambda-1) x_{i t-1}\right) e_{i t} \\
= & \frac{1}{\sqrt{n}} \sum_{i=1}^{n}\left[\frac{1}{T} \sum_{t=1}^{T}\left(\varepsilon_{i t}+(\lambda-1) x_{i t-1}\right) e_{i t}\right]-\sqrt{n} \lim \frac{1}{n T} \sum_{i=1}^{n} \sum_{t=1}^{T} E\left(\varepsilon_{i t}+(\lambda-1) x_{i t-1}\right) e_{i t} \\
= & \frac{1}{\sqrt{n}} \sum_{i=1}^{n}\left[\frac{1}{T} \sum_{t=1}^{T}\left[\left(\varepsilon_{i t}+(\lambda-1) \varepsilon_{i t-1}+\lambda(\lambda-1) \varepsilon_{i t-2}+\lambda^{2}(\lambda-1) \varepsilon_{i t-3}+\cdots\right) e_{i t}\right]\right] \\
& -\sqrt{n} \lim \frac{1}{n T} \sum_{i=1}^{n} \sum_{t=1}^{T} E\left(\varepsilon_{i t}+(\lambda-1) x_{i t-1}\right) e_{i t} \\
\Rightarrow & \frac{1}{\sqrt{n}} \sum_{i=1}^{n}\left[N\left(0, \frac{2 \psi_{00}}{1+\lambda}\right)\right]=N\left(0, \frac{2 \psi_{00}}{1+\lambda}\right)
\end{aligned}
$$

as $(n, T) \stackrel{\text { seq }}{\rightarrow} \infty$, where $\psi_{00}=E\left(\varepsilon_{t}^{2} e_{t}^{2}\right)$.

Consider (3). For part (a), it is easy to see that

$$
\begin{aligned}
& \frac{1}{n T} \sum_{i=1}^{n} \sum_{t=1}^{T}\left(x_{i t}-x_{i t-1}\right)\left(\nu_{i t}-\nu_{i t-1}\right) \\
= & \frac{1}{n} \sum_{i=1}^{n}\left[\frac{1}{T} \sum_{t=1}^{T} \varepsilon_{i t}\left(\nu_{i t}-\nu_{i t-1}\right)\right] \\
& \stackrel{p}{\rightarrow} \lim \frac{1}{n T} \sum_{i=1}^{n} \sum_{t=1}^{T} \varepsilon_{i t}\left(\nu_{i t}-\nu_{i t-1}\right)
\end{aligned}
$$

as $(n, T) \stackrel{\text { seq }}{\rightarrow} \infty$. 
For part (b),

$$
\begin{aligned}
& \frac{1}{\sqrt{n} T} \sum_{i=1}^{n} \sum_{t=1}^{T}\left(x_{i t}-x_{i t-1}\right)\left(\nu_{i t}-\nu_{i t-1}\right)-\sqrt{n} \lim \frac{1}{n T} \sum_{i=1}^{n} \sum_{t=1}^{T} \varepsilon_{i t}\left(\nu_{i t}-\nu_{i t-1}\right) \\
= & \frac{1}{\sqrt{n}} \sum_{i=1}^{n}\left[\frac{1}{T} \sum_{t=1}^{T} \varepsilon_{i t}\left(\nu_{i t}-\nu_{i t-1}\right)\right]-\sqrt{n} \lim \frac{1}{n T} \sum_{i=1}^{n} \sum_{t=1}^{T} \varepsilon_{i t}\left(\nu_{i t}-\nu_{i t-1}\right) \\
= & \frac{1}{\sqrt{n}} \sum_{i=1}^{n}\left[\frac{1}{T} \sum_{t=1}^{T} \varepsilon_{i t}\left(e_{i t}+(\rho-1) \nu_{i t-1}\right)\right]-\sqrt{n} \lim \frac{1}{n T} \sum_{i=1}^{n} \sum_{t=1}^{T} \varepsilon_{i t}\left(\nu_{i t}-\nu_{i t-1}\right) \\
= & \frac{1}{\sqrt{n}} \sum_{i=1}^{n}\left[\frac{1}{T} \sum_{t=1}^{T}\left[\varepsilon_{i t}\left(e_{i t}+(\rho-1) e_{i t-1}+\rho(\rho-1) e_{i t-2}+\rho^{2}(\rho-1) e_{i t-3}+\cdots\right)\right]\right. \\
& -\sqrt{n} \lim \frac{1}{n T} \sum_{i=1}^{n} \sum_{t=1}^{T} \varepsilon_{i t}\left(\nu_{i t}-\nu_{i t-1}\right) \\
\Rightarrow & \frac{1}{\sqrt{n}} \sum_{i=1}^{n}\left[N\left(0, \frac{2 \psi_{00}}{1+\rho}\right)\right]=N\left(0, \frac{2 \psi_{00}}{1+\rho}\right)
\end{aligned}
$$

as $(n, T) \stackrel{\text { seq }}{\rightarrow} \infty$, where $\psi_{00}=E\left(\varepsilon_{t}^{2} e_{t}^{2}\right)$.

Consider (4). For part (a),

$$
\begin{aligned}
& \frac{1}{n T} \sum_{i=1}^{n} \sum_{t=1}^{T}\left(x_{i t}-x_{i t-1}\right)\left(\nu_{i t}-\nu_{i t-1}\right) \\
= & \frac{1}{n} \sum_{i=1}^{n}\left[\frac{1}{T} \sum_{t=1}^{T} \varepsilon_{i t} e_{i t}\right] \\
& \stackrel{p}{\rightarrow} \sigma_{\varepsilon e}
\end{aligned}
$$

as $(n, T) \stackrel{\text { seq }}{\rightarrow} \infty$.

For part (b), note that

$$
\begin{aligned}
& \frac{1}{\sqrt{n T}} \sum_{i=1}^{n} \sum_{t=1}^{T}\left(x_{i t}-x_{i t-1}\right)\left(\nu_{i t}-\nu_{i t-1}\right) \\
= & \frac{1}{\sqrt{n}} \sum_{i=1}^{n}\left[\frac{1}{\sqrt{T}} \sum_{t=1}^{T} \varepsilon_{i t} e_{i t}\right]
\end{aligned}
$$


For a fixed $n$,

$$
\begin{aligned}
& \frac{1}{\sqrt{n T}} \sum_{i=1}^{n} \sum_{t=1}^{T}\left(x_{i t}-x_{i t-1}\right)\left(\nu_{i t}-\nu_{i t-1}\right)-\sqrt{n T} \sigma_{\varepsilon e} \\
= & \frac{1}{\sqrt{n}} \sum_{i=1}^{n}\left[\frac{1}{\sqrt{T}} \sum_{t=1}^{T} \varepsilon_{i t} e_{i t}\right]-\sqrt{n T} \sigma_{\varepsilon e} \\
\Rightarrow & \frac{1}{\sqrt{n}} \sum_{i=1}^{n} Z_{i}
\end{aligned}
$$

as $T \rightarrow \infty$, by a central limit theorem, where $Z_{i} \sim N\left(0, \varpi_{e}^{2} \varpi_{\varepsilon}^{2}\right)$.

$$
\frac{1}{\sqrt{n}} \sum_{i=1}^{n} Z_{i} \Rightarrow N\left(0, \varpi_{e}^{2} \varpi_{\varepsilon}^{2}\right)
$$

as $n \rightarrow \infty$. Hence, we have

$$
\frac{1}{\sqrt{n T}} \sum_{i=1}^{n} \sum_{t=1}^{T}\left(x_{i t}-x_{i t-1}\right)\left(\nu_{i t}-\nu_{i t-1}\right)-\sqrt{n T} \sigma_{\varepsilon e} \Rightarrow N\left(0, \varpi_{e}^{2} \varpi_{\varepsilon}^{2}\right)
$$

as $(n, T) \stackrel{\text { seq }}{\rightarrow} \infty$.

Proof of Theorem 3:

Proof. By $\widehat{\beta}_{F D}-\beta=\frac{\sum_{i=1}^{n} \sum_{t=1}^{T}\left(x_{i t}-x_{i t-1}\right)\left(\nu_{i t}-\nu_{i t-1}\right)}{\sum_{i=1}^{n} \sum_{t=1}^{T}\left(x_{i t}-x_{i t-1}\right)^{2}}$, the proof of Theorem 3 is straightforward with above lemmas.

\section{F Proof of Theorem 4}

Define $\mathbf{z}=\left[\boldsymbol{\iota}_{n T}, \mathbf{x}\right]$, then

$$
\begin{aligned}
\left(\begin{array}{c}
\hat{\alpha}_{G L S} \\
\hat{\beta}_{G L S}
\end{array}\right) & =\left(\mathbf{z}^{\prime} \Phi^{-1} \mathbf{z}\right)^{-1}\left(\mathbf{z}^{\prime} \Phi^{-1} \mathbf{y}\right) \\
& =\left(\left[\begin{array}{c}
\boldsymbol{\iota}_{n T}^{\prime} \\
\mathbf{x}^{\prime}
\end{array}\right] \Phi^{-1}\left[\begin{array}{ll}
\boldsymbol{\iota}_{n T} & \mathbf{x}
\end{array}\right]\right)^{-1}\left(\left[\begin{array}{c}
\boldsymbol{\iota}_{n T}^{\prime} \\
\mathbf{x}^{\prime}
\end{array}\right] \Phi^{-1} \mathbf{y}\right) \\
& =\left[\begin{array}{cc}
\boldsymbol{\iota}_{n T}^{\prime} \Phi^{-1} \boldsymbol{\iota}_{n T} & \boldsymbol{\iota}_{n T}^{\prime} \Phi^{-1} \mathbf{x} \\
\mathbf{x}^{\prime} \Phi^{-1} \boldsymbol{\iota}_{n T} & \mathbf{x}^{\prime} \Phi^{-1} \mathbf{x}
\end{array}\right]^{-1}\left[\begin{array}{c}
\boldsymbol{\iota}_{n T}^{\prime} \Phi^{-1} \mathbf{y} \\
\mathbf{x}^{\prime} \Phi^{-1} \mathbf{y}
\end{array}\right] \\
& =\left[\begin{array}{cc}
F_{11} & F_{12} \\
F_{21} & F_{22}
\end{array}\right]\left[\begin{array}{c}
\boldsymbol{\iota}_{n T}^{\prime} \Phi^{-1} \mathbf{y} \\
\mathbf{x}^{\prime} \Phi^{-1} \mathbf{y}
\end{array}\right] \\
& =\left[\begin{array}{c}
F_{11} \boldsymbol{\iota}_{n T}^{\prime} \Phi^{-1} \mathbf{y}+F_{12} \mathbf{x}^{\prime} \Phi^{-1} \mathbf{y} \\
F_{21} \boldsymbol{\iota}_{n T}^{\prime} \Phi^{-1} \mathbf{y}+F_{22} \mathbf{x}^{\prime} \Phi^{-1} \mathbf{y}
\end{array}\right]
\end{aligned}
$$


where

$$
\begin{aligned}
& F_{11}=\left[\boldsymbol{\iota}_{n T}^{\prime} \Phi^{-1} \iota_{n T}-\boldsymbol{\iota}_{n T}^{\prime} \Phi^{-1} \mathbf{x}\left(\mathbf{x}^{\prime} \Phi^{-1} \mathbf{x}\right)^{-1} \mathbf{x}^{\prime} \Phi^{-1} \iota_{n T}\right]^{-1} \\
& F_{12}=-\left(\boldsymbol{\iota}_{n T}^{\prime} \Phi^{-1} \iota_{n T}\right)^{-1} \boldsymbol{\iota}_{n T}^{\prime} \Phi^{-1} \mathbf{x}\left[\mathbf{x}^{\prime} \Phi^{-1} \mathbf{x}-\mathbf{x}^{\prime} \Phi^{-1} \iota_{n T}\left(\boldsymbol{\iota}_{n T}^{\prime} \Phi^{-1} \iota_{n T}\right)^{-1} \boldsymbol{\iota}_{n T}^{\prime} \Phi^{-1} \mathbf{x}\right]^{-1}, \\
& F_{21}=-\left[\mathbf{x}^{\prime} \Phi^{-1} \mathbf{x}-\mathbf{x}^{\prime} \Phi^{-1} \iota_{n T}\left(\boldsymbol{\iota}_{n T}^{\prime} \Phi^{-1} \iota_{n T}\right)^{-1} \boldsymbol{\iota}_{n T}^{\prime} \Phi^{-1} \mathbf{x}\right]^{-1} \mathbf{x}^{\prime} \Phi^{-1} \iota_{n T}\left(\boldsymbol{\iota}_{n T}^{\prime} \Phi^{-1} \iota_{n T}\right)^{-1}, \\
& F_{22}=\left[\mathbf{x}^{\prime} \Phi^{-1} \mathbf{x}-\mathbf{x}^{\prime} \Phi^{-1} \iota_{n T}\left(\boldsymbol{\iota}_{n T}^{\prime} \Phi^{-1} \iota_{n T}\right)^{-1} \boldsymbol{\iota}_{n T}^{\prime} \Phi^{-1} \mathbf{x}\right]^{-1} .
\end{aligned}
$$

Hence

$$
\begin{aligned}
\widehat{\beta}_{G L S} & =F_{21} \boldsymbol{\iota}_{n T}^{\prime} \Phi^{-1} \mathbf{y}+F_{22}^{\prime} \mathbf{x}^{\prime} \Phi^{-1} \mathbf{y} \\
& =\left[\mathbf{x}^{\prime} \Phi^{-1} \mathbf{x}-\mathbf{x}^{\prime} \Phi^{-1} \iota_{n T}\left(\boldsymbol{\iota}_{n T}^{\prime} \Phi^{-1} \iota_{n T}\right)^{-1} \boldsymbol{\iota}_{n T}^{\prime} \Phi^{-1} \mathbf{x}\right]^{-1}\left[\mathbf{x}^{\prime} \Phi^{-1} \mathbf{y}-\mathbf{x}^{\prime} \Phi^{-1} \iota_{n T}\left(\boldsymbol{\iota}_{n T}^{\prime} \Phi^{-1} \iota_{n T}\right)^{-1} \boldsymbol{\iota}_{n T}^{\prime} \Phi^{-1} \mathbf{y}\right]
\end{aligned}
$$

and

$$
\widehat{\beta}_{G L S}-\beta=G_{1}^{-1} G_{2},
$$

where $G_{1}=\mathbf{x}^{\prime} \Phi^{-1} \mathbf{x}-\mathbf{x}^{\prime} \Phi^{-1} \iota_{n T}\left(\boldsymbol{\iota}_{N T}^{\prime} \Phi^{-1} \iota_{n T}\right)^{-1} \boldsymbol{\iota}_{N T}^{\prime} \Phi^{-1} \mathbf{x}$ and $G_{2}=\mathbf{x}^{\prime} \Phi^{-1} \mathbf{u}-$ $\mathbf{x}^{\prime} \Phi^{-1} \iota_{n T}\left(\iota_{N T}^{\prime} \Phi^{-1} \iota_{n T}\right)^{-1} \iota_{N T}^{\prime} \Phi^{-1} \mathbf{u}$.

With the definition of $\Phi$, we have

$$
\begin{aligned}
\mathbf{x}^{\prime} \Phi^{-1} \mathbf{x} & =\frac{1}{\varpi_{e}^{2}} \sum_{i=1}^{n}\left(\mathbf{x}_{i}^{\prime} \mathbf{A}_{i}^{-1} \mathbf{x}_{i}-\frac{\sigma_{\mu}^{2}}{\varpi_{e}^{2}+\theta \sigma_{\mu}^{2}} \mathbf{x}_{i}^{\prime} \mathbf{A}^{-1} \iota_{T} \iota_{T}^{\prime} \mathbf{A}_{i}^{-1} \mathbf{x}_{i}\right) \\
\mathbf{x}^{\prime} \Phi^{-1} \iota_{n T} & =\frac{1}{\varpi_{e}^{2}} \sum_{i=1}^{n}\left(\mathbf{x}_{i}^{\prime} \mathbf{A}^{-1} \iota_{T}-\frac{\sigma_{\mu}^{2}}{\varpi_{e}^{2}+\theta \sigma_{\mu}^{2}} \mathbf{x}_{i}^{\prime} \mathbf{A}^{-1} \iota_{T} \iota_{T}^{\prime} \mathbf{A}_{i}^{-1} \mathbf{x}_{i}\right) \\
& =\frac{1}{\varpi_{e}^{2}} \sum_{i=1}^{n}\left(\mathbf{x}_{i}^{\prime} \mathbf{A}^{-1} \iota_{T}-\frac{\theta \sigma_{\mu}^{2}}{\varpi_{e}^{2}+\theta \sigma_{\mu}^{2}} \mathbf{x}_{i}^{\prime} \mathbf{A}^{-1} \iota_{T}\right) \\
& =\frac{1}{\varpi_{e}^{2}+\theta \sigma_{\mu}^{2}} \sum_{i=1}^{n}\left(\mathbf{x}_{i}^{\prime} \mathbf{A}^{-1} \iota_{T}\right),
\end{aligned}
$$




$$
\begin{aligned}
& \boldsymbol{\iota}_{n T}^{\prime} \Phi^{-1} \iota_{n T}=\frac{1}{\varpi_{e}^{2}} \sum_{i=1}^{n}\left(\iota_{T}^{\prime} \mathbf{A}^{-1} \iota_{T}-\frac{\sigma_{\mu}^{2}}{\varpi_{e}^{2}+\theta \sigma_{\mu}^{2}} \boldsymbol{\iota}_{T}^{\prime} \mathbf{A}^{-1} \iota_{T} \iota_{T}^{\prime} \mathbf{A}^{-1} \iota_{T}\right) \\
& =\frac{1}{\varpi_{e}^{2}} \sum_{i=1}^{n}\left(\theta-\frac{\sigma_{\mu}^{2}}{\varpi_{e}^{2}+\theta \sigma_{\mu}^{2}} \theta^{2}\right) \\
& =\frac{1}{\varpi_{e}^{2}} n \theta\left(1-\frac{\theta \sigma_{\mu}^{2}}{\varpi_{e}^{2}+\theta \sigma_{\mu}^{2}}\right) \\
& =\frac{n \theta}{\varpi_{e}^{2}+\theta \sigma_{\mu}^{2}}, \\
& \mathbf{x}^{\prime} \Phi^{-1} \mathbf{u}=\frac{1}{\varpi_{e}^{2}} \sum_{i=1}^{n}\left(\mathbf{x}_{i}^{\prime} \mathbf{A}^{-1} \mathbf{u}_{i}-\frac{\sigma_{\mu}^{2}}{\varpi_{e}^{2}+\theta \sigma_{\mu}^{2}} \mathbf{x}_{i}^{\prime} \mathbf{A}^{-1} \iota_{T} \iota_{T}^{\prime} A^{-1} \mathbf{u}_{i}\right) \\
& =\frac{1}{\varpi_{e}^{2}} \sum_{i=1}^{n}\left[\left(\mu_{i} \mathbf{x}_{i}^{\prime} \mathbf{A}^{-1} \iota_{T}-\frac{\mu_{i} \sigma_{\mu}^{2}}{\varpi_{e}^{2}+\theta \sigma_{\mu}^{2}} \mathbf{x}_{i}^{\prime} \mathbf{A}^{-1} \iota_{T} \iota_{T}^{\prime} \mathbf{A}^{-1} \iota_{T}\right)+\left(\mathbf{x}_{i}^{\prime} \mathbf{A}^{-1} \boldsymbol{\nu}_{i}-\frac{\sigma_{\mu}^{2}}{\varpi_{e}^{2}+\theta \sigma_{\mu}^{2}} \mathbf{x}_{i}^{\prime} \mathbf{A}^{-1} \iota_{T} \iota_{T}^{\prime} \mathbf{A}^{-}\right.\right. \\
& =\frac{1}{\varpi_{e}^{2}} \sum_{i=1}^{n}\left[\left(\mu_{i} \mathbf{x}_{i}^{\prime} \mathbf{A}^{-1} \iota_{T}-\frac{\mu_{i} \theta \sigma_{\mu}^{2}}{\varpi_{e}^{2}+\theta \sigma_{\mu}^{2}} \mathbf{x}_{i}^{\prime} \mathbf{A}^{-1} \iota_{T}\right)+\left(\mathbf{x}_{i}^{\prime} \mathbf{A}^{-1} \boldsymbol{\nu}_{i}-\frac{\sigma_{\mu}^{2}}{\varpi_{e}^{2}+\theta \sigma_{\mu}^{2}} \mathbf{x}_{i}^{\prime} \mathbf{A}^{-1} \iota_{T} \boldsymbol{\iota}_{T}^{\prime} \mathbf{A}^{-1} \boldsymbol{\nu}_{i}\right)\right] \\
& =\frac{1}{\varpi_{e}^{2}} \sum_{i=1}^{n}\left[\frac{\mu_{i} \sigma_{e}^{2}}{\sigma_{e}^{2}+\theta \sigma_{\mu}^{2}} \mathbf{x}_{i}^{\prime} \mathbf{A}^{-1} \iota_{T}+\left(\mathbf{x}_{i}^{\prime} \mathbf{A}^{-1} \boldsymbol{\nu}_{i}-\frac{\sigma_{\mu}^{2}}{\varpi_{e}^{2}+\theta \sigma_{\mu}^{2}} \mathbf{x}_{i}^{\prime} \mathbf{A}^{-1} \iota_{T} \boldsymbol{\iota}_{T}^{\prime} \mathbf{A}^{-1} \boldsymbol{\nu}_{i}\right)\right], \\
& \boldsymbol{\iota}_{n T}^{\prime} \Phi^{-1} \mathbf{u}=\frac{1}{\sigma_{e}^{2}} \sum_{i=1}^{n}\left(\iota_{T}^{\prime} \mathbf{A}^{-1} \mathbf{u}_{i}-\frac{\sigma_{\mu}^{2}}{\varpi_{e}^{2}+\theta \sigma_{\mu}^{2}} \boldsymbol{\iota}_{T}^{\prime} \mathbf{A}^{-1} \iota_{T} \iota_{T}^{\prime} \mathbf{A}^{-1} \mathbf{u}_{i}\right) \\
& =\frac{1}{\sigma_{e}^{2}} \sum_{i=1}^{n}\left[\left(\mu_{i} \iota_{T}^{\prime} \mathbf{A}^{-1} \iota_{T}-\frac{\mu_{i} \sigma_{\mu}^{2}}{\varpi_{e}^{2}+\theta \sigma_{\mu}^{2}} \iota_{T}^{\prime} \mathbf{A}^{-1} \iota_{T} \iota_{T}^{\prime} \mathbf{A}^{-1} \iota_{T}\right)+\left(\iota_{T}^{\prime} \mathbf{A}^{-1} \boldsymbol{\nu}_{i}-\frac{\sigma_{\mu}^{2}}{\varpi_{e}^{2}+\theta \sigma_{\mu}^{2}} \iota_{T}^{\prime} \mathbf{A}^{-1} \iota_{T} \boldsymbol{\iota}_{T}^{\prime} \mathbf{A}\right.\right. \\
& =\frac{1}{\sigma_{e}^{2}} \sum_{i=1}^{n}\left[\left(\mu_{i} \theta-\frac{\mu_{i} \theta^{2} \sigma_{\mu}^{2}}{\varpi_{e}^{2}+\theta \sigma_{\mu}^{2}}\right)+\left(\iota_{T}^{\prime} \mathbf{A}^{-1} \boldsymbol{\nu}_{i}-\frac{\theta \sigma_{\mu}^{2}}{\varpi_{e}^{2}+\theta \sigma_{\mu}^{2}} \boldsymbol{\iota}_{T}^{\prime} \mathbf{A}^{-1} \boldsymbol{\nu}_{i}\right)\right] \\
& =\frac{1}{\sigma_{e}^{2}} \sum_{i=1}^{n}\left[\frac{\mu_{i} \theta \varpi_{e}^{2}}{\varpi_{e}^{2}+\theta \sigma_{\mu}^{2}}+\frac{\theta \varpi_{e}^{2}}{\varpi_{e}^{2}+\theta \sigma_{\mu}^{2}} \iota_{T}^{\prime} \mathbf{A}^{-1} \boldsymbol{\nu}_{i}\right] \\
& =\sum_{i=1}^{n}\left[\frac{\theta}{\varpi_{e}^{2}+\theta \sigma_{\mu}^{2}}\left(\mu_{i}+\boldsymbol{\iota}_{T}^{\prime} \mathbf{A}^{-1} \boldsymbol{\nu}_{i}\right)\right]
\end{aligned}
$$

because $\mathbf{u}_{i}=\mu_{i} \iota_{T}+\boldsymbol{\nu}_{i}$.

$$
\text { When }|\rho|<1, \mathbf{A}=\frac{1}{1-\rho^{2}}\left[\begin{array}{ccccc}
1 & \rho & \rho^{2} & \cdots & \rho^{T-1} \\
\rho & 1 & \rho & \cdots & \rho^{T-2} \\
\rho^{2} & \rho & 1 & \cdots & \rho^{T-3} \\
\vdots & \vdots & \vdots & \ddots & \vdots \\
\rho^{T-1} & \rho^{T-2} & \rho^{T-3} & \cdots & 1
\end{array}\right], \mathbf{A}^{-1}=\left[\begin{array}{cccccc}
1 & -\rho & 0 & 0 & \cdots & 0 \\
-\rho & 1+\rho^{2} & -\rho & 0 & \cdots & 0 \\
0 & -\rho & 1+\rho^{2} & -\rho & \cdots & 0 \\
\vdots & \vdots & \vdots & \vdots & \ddots & \vdots \\
0 & 0 & 0 & \cdots & -\rho & 1+ \\
0 & 0 & 0 & \cdots & 0 & -\rho
\end{array}\right.
$$

27 
It can be shown $\mathbf{A}^{-1}=\mathbf{C C}^{\prime}$, where $\mathbf{C}=\left[\begin{array}{cccccc}\sqrt{1-\rho^{2}} & 0 & 0 & \cdots & 0 & 0 \\ -\rho & 1 & 0 & \cdots & 0 & 0 \\ 0 & -\rho & 1 & \cdots & 0 & 0 \\ \vdots & \vdots & \vdots & \ddots & \vdots & \vdots \\ 0 & 0 & 0 & -\rho & 1 & 0 \\ 0 & 0 & 0 & 0 & -\rho & 1\end{array}\right]$ is the Prais-Winsten (PW) transformation matrix suggested in Baltagi and $\mathrm{Li}$ (1991). Hence

$$
\begin{aligned}
& \mathbf{C x}_{i}=\left[\begin{array}{c}
\sqrt{1-\rho^{2}} x_{i 1}^{\prime} \\
x_{i 2}^{\prime}-\rho x_{i 1}^{\prime} \\
x_{i 3}^{\prime}-\rho x_{i 2}^{\prime} \\
\vdots \\
x_{i T-1}^{\prime}-\rho x_{i T-2}^{\prime} \\
x_{i T}^{\prime}-\rho x_{i T-1}^{\prime}
\end{array}\right], \mathbf{C} \boldsymbol{\nu}_{i}=\left[\begin{array}{c}
\sqrt{1-\rho^{2}} \nu_{i 1}^{\prime} \\
\nu_{i 2}^{\prime}-\rho \nu_{i 1}^{\prime} \\
\nu_{i 3}^{\prime}-\rho \nu_{i 2}^{\prime} \\
\vdots \\
\nu_{i T-1}^{\prime}-\rho \nu_{i T-2}^{\prime} \\
\nu_{i T}^{\prime}-\rho \nu_{i T-1}^{\prime}
\end{array}\right]=\left[\begin{array}{c}
\sqrt{1-\rho^{2}} e_{i 1}^{\prime} \\
e_{i 2}^{\prime} \\
e_{i 3}^{\prime} \\
\vdots \\
e_{i T-1}^{\prime} \\
e_{i T}^{\prime}
\end{array}\right] \\
& \mathbf{C}_{T}=\left[\begin{array}{c}
\sqrt{1-\rho^{2}} \\
1-\rho \\
1-\rho \\
\vdots \\
1-\rho \\
1-\rho
\end{array}\right] . \text { And } \\
& \mathbf{x}_{i}^{\prime} \mathbf{A}^{-1} \mathbf{x}_{i}=\mathbf{x}_{i}^{\prime} \mathbf{C}^{\prime} \mathbf{C} \mathbf{x}_{i} \approx \sum_{t=1}^{T}\left(x_{i t}-\rho x_{i t-1}\right)^{2} \\
& \mathbf{x}_{i}^{\prime} \mathbf{A}^{-1} \boldsymbol{\nu}_{i}=\mathbf{x}_{i}^{\prime} \mathbf{C}^{\prime} \mathbf{C} \boldsymbol{\nu}_{i} \approx \sum_{t=1}^{T}\left(x_{i t}-\rho x_{i t-1}\right) e_{i t} \\
& \mathbf{x}_{i}^{\prime} \mathbf{A}^{-1} \iota_{T}=\mathbf{x}_{i}^{\prime} \mathbf{C}^{\prime} \mathbf{C} \iota_{T} \approx(1-\rho) \sum_{t=1}^{T}\left(x_{i t}-\rho x_{i t-1}\right) \\
& \boldsymbol{\iota}_{T}^{\prime} \mathbf{A}^{-1} \boldsymbol{\nu}_{i}=\boldsymbol{\iota}_{T}^{\prime} \mathbf{C}^{\prime} \mathbf{C} \boldsymbol{\iota}_{T} \boldsymbol{\nu}_{i} \approx(1-\rho) \sum_{t=1}^{T} e_{i t} \\
& \theta=\iota_{T}^{\prime} \mathbf{A}^{-1} \iota_{T}=\iota_{T}^{\prime} \mathbf{C}^{\prime} \mathbf{C}_{T} \approx \sum_{t=1}^{T}(1-\rho)^{2}=O\left((1-\rho)^{2} T\right) . \\
& \text { When } \rho=1, \mathbf{A}=\left[\begin{array}{ccccc}
1 & 1 & 1 & \cdots & 1 \\
1 & 2 & 2 & \cdots & 2 \\
1 & 2 & 3 & \cdots & 3 \\
\vdots & \vdots & \vdots & \ddots & \vdots \\
1 & 2 & 3 & \cdots & T
\end{array}\right], \mathbf{A}^{-1}=\left[\begin{array}{ccccc}
2 & -1 & 0 & \cdots & 0 \\
-1 & 2 & -1 & \cdots & 0 \\
0 & -1 & 2 & \cdots & \vdots \\
\vdots & \vdots & \vdots & \ddots & -1 \\
0 & 0 & 0 & -1 & 1
\end{array}\right] \text {. }
\end{aligned}
$$


It can be shown $\mathbf{A}^{-1}=\mathbf{C C}^{\prime}$, where $\mathbf{C}=\left[\begin{array}{cccccc}1 & 0 & 0 & \cdots & 0 & 0 \\ -1 & 1 & 0 & \cdots & 0 & 0 \\ 0 & -1 & 1 & \cdots & 0 & 0 \\ \vdots & \vdots & \vdots & \ddots & \vdots & \vdots \\ 0 & 0 & 0 & -1 & 1 & 0 \\ 0 & 0 & 0 & 0 & -1 & 1\end{array}\right]$.

Hence

$$
\mathbf{C x}_{i}=\left[\begin{array}{c}
x_{i 1}^{\prime} \\
x_{i 2}^{\prime}-x_{i 1}^{\prime} \\
x_{i 3}^{\prime}-x_{i 2}^{\prime} \\
\vdots \\
x_{i T-1}^{\prime}-x_{i T-2}^{\prime} \\
x_{i T}^{\prime}-x_{i T-1}^{\prime}
\end{array}\right], \mathbf{C} \boldsymbol{\nu}_{i}=\left[\begin{array}{c}
\nu_{i 1}^{\prime} \\
\nu_{i 2}^{\prime}-\nu_{i 1}^{\prime} \\
\nu_{i 3}^{\prime}-\nu_{i 2}^{\prime} \\
\vdots \\
\nu_{i T-1}^{\prime}-\nu_{i T-2}^{\prime} \\
\nu_{i T}^{\prime}-\nu_{i T-1}^{\prime}
\end{array}\right]=\left[\begin{array}{c}
e_{i 1}^{\prime} \\
e_{i 2}^{\prime} \\
e_{i 3}^{\prime} \\
\vdots \\
e_{i T-1}^{\prime} \\
e_{i T}^{\prime}
\end{array}\right], \mathbf{C} \boldsymbol{\iota}_{T}=\left[\begin{array}{c}
1 \\
0 \\
0 \\
\vdots \\
0 \\
0
\end{array}\right] .
$$

And

$$
\begin{gathered}
\mathbf{x}_{i}^{\prime} \mathbf{A}^{-1} \mathbf{x}_{i}=\mathbf{x}_{i}^{\prime} \mathbf{C}^{\prime} \mathbf{C} \mathbf{x}_{i} \approx \sum_{t=1}^{T}\left(x_{i t}-x_{i t-1}\right)^{2} \\
\mathbf{x}_{i}^{\prime} \mathbf{A}^{-1} \boldsymbol{\nu}_{i}=\mathbf{x}_{i}^{\prime} \mathbf{C}^{\prime} \mathbf{C} \boldsymbol{\nu}_{i} \approx \sum_{t=1}^{T}\left(x_{i t}-x_{i t-1}\right) e_{i t} \\
\mathbf{x}_{i}^{\prime} \mathbf{A}^{-1} \iota_{T}=\mathbf{x}_{i}^{\prime} \mathbf{C}^{\prime} \mathbf{C} \boldsymbol{\iota}_{T}=x_{i 1}^{\prime} \\
\boldsymbol{\iota}_{T}^{\prime} \mathbf{A}^{-1} \boldsymbol{\nu}_{i}=\boldsymbol{\iota}_{T}^{\prime} \mathbf{C}^{\prime} \mathbf{C} \iota_{T} \boldsymbol{\nu}_{i}=\nu_{i 1}^{\prime} \\
\theta=\boldsymbol{\iota}_{T}^{\prime} \mathbf{A}^{-1} \iota_{T}=\iota_{T}^{\prime} \mathbf{C}^{\prime} \mathbf{C} \iota_{T}=1
\end{gathered}
$$

The following lemmas will be used to prove Theorem 4 .

Lemma 7 If Assumptions $1-2$ hold, then

1. If $|\rho|<1$ and $|\lambda|<1$,
(a) $\frac{1}{n T} G_{1} \stackrel{p}{\rightarrow} \frac{1}{\varpi_{e}^{2}}\left[\frac{\left(1-2 \rho \lambda+\rho^{2}\right) \sigma_{\varepsilon}^{2}}{1-\lambda^{2}}+\frac{2\left(\lambda-2 \rho \lambda^{2}+\rho^{2} \lambda-\rho\right) \gamma_{\varepsilon}^{2}}{1-\lambda}\right]$,
(b) $\frac{1}{n T} G_{2} \stackrel{p}{\rightarrow} \frac{1}{\varpi_{e}^{2}} \lim \frac{1}{n} \sum_{i=1}^{n} \frac{1}{T} \sum_{t=1}^{T} E\left[\left(x_{i t}-\rho x_{i t-1}\right) e_{i t}\right]$, 
(c) $\frac{1}{\sqrt{n T}} G_{2}-\sqrt{n T} \frac{1}{\varpi_{e}^{2}} \lim \frac{1}{n} \sum_{i=1}^{n} \frac{1}{T} \sum_{t=1}^{T} E\left[\left(x_{i t}-\rho x_{i t-1}\right) e_{i t}\right] \Rightarrow \frac{1}{\varpi_{e}^{2}} N\left(0, \frac{\left(1-2 \rho \lambda+\rho^{2}\right) \psi_{00}}{1-\lambda^{2}}\right)$.

2. If $\rho=1$ and $|\lambda|<1$,
(a) $\frac{1}{n T} G_{1} \stackrel{p}{\rightarrow} \frac{1}{\varpi_{e}^{2}}\left[\frac{2 \sigma_{\varepsilon}^{2}}{1+\lambda}+\frac{2\left(-2 \lambda^{2}+2 \lambda-1\right) \gamma_{\varepsilon}^{2}}{1-\lambda}\right]$,
(b) $\frac{1}{n T} G_{2} \stackrel{p}{\rightarrow} \frac{1}{\varpi_{e}^{2}} \lim \frac{1}{n} \sum_{i=1}^{n} \frac{1}{T} \sum_{t=1}^{T} E\left[\left(x_{i t}-x_{i t-1}\right) e_{i t}\right]$,
(c) $\frac{1}{\sqrt{n T}} G_{2}-\sqrt{n T} \frac{1}{\varpi_{e}^{2}} \lim \frac{1}{n} \sum_{i=1}^{n} \frac{1}{T} \sum_{t=1}^{T} E\left[\left(x_{i t}-x_{i t-1}\right) e_{i t}\right] \Rightarrow \frac{1}{\varpi_{e}^{2}} N\left(0, \frac{2 \psi_{00}}{1+\lambda}\right)$.

3. If $|\rho|<1$ and $\lambda=1$,
(a) $\frac{1}{n T^{2}} G_{1} \stackrel{p}{\rightarrow} \frac{(1-\rho)^{2} \varpi_{\varepsilon}^{2}}{6 \varpi_{e}^{2}}$,
(b) $\frac{1}{n T} G_{2} \stackrel{p}{\rightarrow} \frac{1}{\varpi_{e}^{2}}\left[(1-\rho)\left[-\frac{1}{2} \varpi_{\varepsilon e}+\gamma_{\varepsilon e}\right]+\sigma_{\varepsilon e}\right]$,
(c) $\frac{1}{\sqrt{n} T} G_{2}-\frac{1}{\varpi_{e}^{2}} \frac{1}{\sqrt{n}} \sum_{i=1}^{n}(1-\rho)\left[\left(\frac{1}{T} \sum_{t=1}^{T}\left(x_{i t}-\bar{x}_{i}\right) \varepsilon_{i t}\right) \frac{\varpi_{\varepsilon e}}{\varpi_{\varepsilon}^{2}}+\gamma_{\varepsilon e}+\frac{\sigma_{\varepsilon e}}{1-\rho}\right] \Rightarrow$ $N\left(0, \frac{(1-\rho)^{2} \varpi_{\varepsilon}^{2} \varpi_{e . \varepsilon}}{6 \varpi_{e}^{4}}\right)$,

4. If $\rho=1$ and $\lambda=1$,
(a) $\frac{1}{n T} G_{1} \stackrel{p}{\rightarrow} \frac{\sigma_{\varepsilon}^{2}}{\varpi_{e}^{2}}$,
(b) $\frac{1}{n T} G_{2} \stackrel{p}{\rightarrow} \frac{\sigma_{\varepsilon e}}{\varpi_{e}^{2}}$
(c) $\frac{1}{\sqrt{n T}} G_{2}-\sqrt{n T} \frac{\sigma_{\varepsilon e}}{\varpi_{e}^{2}} \Rightarrow \frac{1}{\varpi_{e}^{2}} N\left(0, \varpi_{e}^{2} \varpi_{\varepsilon}^{2}\right)$.

\section{Proof.}

Note that

$$
\frac{1}{n T} G_{1}=\frac{1}{n T} \mathbf{x}^{\prime} \Phi^{-1} \mathbf{x}-\frac{1}{T} \frac{\mathbf{x}^{\prime} \Phi^{-1} \iota_{n T}}{n}\left(\frac{\boldsymbol{\iota}_{n T}^{\prime} \Phi^{-1} \iota_{n T}}{n}\right)^{-1} \frac{\boldsymbol{\iota}_{n T}^{\prime} \Phi^{-1} \mathbf{x}}{n}
$$

First consider

$$
\begin{aligned}
& \frac{1}{n T} \mathbf{x}^{\prime} \Phi^{-1} \mathbf{x} \\
= & \frac{1}{\varpi_{e}^{2}} \frac{1}{n} \sum_{i=1}^{n}\left(\frac{1}{T} \mathbf{x}_{i}^{\prime} \mathbf{A}^{-1} \mathbf{x}_{i}-\frac{T \sigma_{\mu}^{2}}{\varpi_{e}^{2}+\theta \sigma_{\mu}^{2}} \frac{\mathbf{x}_{i}^{\prime} \mathbf{A}^{-1} \iota_{T}}{T} \frac{\iota_{T}^{\prime} \mathbf{A}^{-1} \mathbf{x}_{i}}{T}\right)
\end{aligned}
$$


For a fixed $n$,

$$
\begin{aligned}
& \frac{1}{T} \mathbf{x}_{i}^{\prime} \mathbf{A}^{-1} \mathbf{x}_{i} \\
= & \frac{1}{T} \sum_{t=1}^{T}\left(x_{i t}-\rho x_{i t-1}\right)^{2} \\
= & \frac{1}{T} \sum_{t=1}^{T} x_{i t}^{2}+\rho^{2} \frac{1}{T} \sum_{t=1}^{T} x_{i t-1}^{2}-\rho \frac{1}{T} \sum_{t=1}^{T} 2 x_{i t-1} x_{i t} \\
& \stackrel{p}{\rightarrow}\left(1+\rho^{2}\right)\left(\frac{\sigma_{\varepsilon}^{2}}{1-\lambda^{2}}+\frac{2 \lambda \gamma_{\varepsilon}^{2}}{1-\lambda}\right)-2 \rho\left[\lambda\left(\frac{\sigma_{\varepsilon}^{2}}{1-\lambda^{2}}+\frac{2 \lambda \gamma_{\varepsilon}^{2}}{1-\lambda}\right)+\frac{\gamma_{\varepsilon}^{2}}{1-\lambda}\right] \\
= & \frac{\left(1-2 \rho \lambda+\rho^{2}\right) \sigma_{\varepsilon}^{2}}{1-\lambda^{2}}+\frac{2\left(\lambda-2 \rho \lambda^{2}+\rho^{2} \lambda-\rho\right) \gamma_{\varepsilon}^{2}}{1-\lambda}
\end{aligned}
$$

by a CLT and also

$$
\frac{1}{T} \mathbf{x}_{i}^{\prime} \mathbf{A}^{-1} \iota_{T}=(1-\rho) \frac{1}{T} \sum_{t=1}^{T}\left(x_{i t}-\rho x_{i t-1}\right) \stackrel{p}{\rightarrow} 0
$$

as $T \rightarrow \infty$. So, with $\theta=O\left((1-\rho)^{2} T\right)$ when $|\rho|<1$,

$$
\begin{aligned}
& \frac{1}{n T} \mathbf{x}^{\prime} \Phi^{-1} \mathbf{x} \\
= & \frac{1}{\varpi_{e}^{2}} \frac{1}{n} \sum_{i=1}^{n}\left(\frac{1}{T} \mathbf{x}_{i}^{\prime} \mathbf{A}^{-1} \mathbf{x}_{i}-\frac{T \sigma_{\mu}^{2}}{\varpi_{e}^{2}+\theta \sigma_{\mu}^{2}} \frac{\mathbf{x}_{i}^{\prime} \mathbf{A}^{-1} \iota_{T}}{T} \frac{\iota_{T}^{\prime} \mathbf{A}^{-1} \mathbf{x}_{i}}{T}\right) \\
& \stackrel{p}{\rightarrow} \frac{1}{\varpi_{e}^{2}} \frac{1}{n} \sum_{i=1}^{n}\left[\frac{\left(1-2 \rho \lambda+\rho^{2}\right) \sigma_{\varepsilon}^{2}}{1-\lambda^{2}}+\frac{2\left(\lambda-2 \rho \lambda^{2}+\rho^{2} \lambda-\rho\right) \gamma_{\varepsilon}^{2}}{1-\lambda}\right] \\
= & \frac{1}{\varpi_{e}^{2}}\left[\frac{\left(1-2 \rho \lambda+\rho^{2}\right) \sigma_{\varepsilon}^{2}}{1-\lambda^{2}}+\frac{2\left(\lambda-2 \rho \lambda^{2}+\rho^{2} \lambda-\rho\right) \gamma_{\varepsilon}^{2}}{1-\lambda}\right]
\end{aligned}
$$

holds for all $n$ and hence it holds for a large $n$ as well.

Also because

$$
\frac{1}{n} \mathbf{x}^{\prime} \Phi^{-1} \iota_{n T}=\frac{1}{n} \sum_{i=1}^{n} \frac{1}{\sigma_{e}^{2}+\theta \sigma_{\mu}^{2}}\left(\mathbf{x}_{i}^{\prime} \mathbf{A}^{-1} \iota_{T}\right) \stackrel{p}{\rightarrow} 0
$$

because $\theta=O(T)$ and $\frac{1}{T} \mathbf{x}_{i}^{\prime} \mathbf{A}^{-1} \iota_{T} \stackrel{p}{\rightarrow} 0$. And

$$
\frac{1}{n} \boldsymbol{\iota}_{n T}^{\prime} \Phi^{-1} \iota_{n T}=\frac{1}{n} \frac{n \theta}{\varpi_{e}^{2}+\theta \sigma_{\mu}^{2}} \stackrel{p}{\rightarrow} \frac{1}{\sigma_{\mu}^{2}}
$$


Hence, we have

$$
\begin{aligned}
\frac{1}{n T} G_{1}= & \frac{1}{n T} \mathbf{x}^{\prime} \Phi^{-1} \mathbf{x}-\frac{1}{T} \frac{\mathbf{x}^{\prime} \Phi^{-1} \boldsymbol{\iota}_{n T}}{n}\left(\frac{\boldsymbol{\iota}_{n T}^{\prime} \Phi^{-1} \boldsymbol{\iota}_{n T}}{n}\right)^{-1} \frac{\boldsymbol{\iota}_{n T}^{\prime} \Phi^{-1} \mathbf{x}}{n} \\
& \stackrel{p}{\rightarrow} \frac{1}{\varpi_{e}^{2}}\left[\frac{\left(1-2 \rho \lambda+\rho^{2}\right) \sigma_{\varepsilon}^{2}}{1-\lambda^{2}}+\frac{2\left(\lambda-2 \rho \lambda^{2}+\rho^{2} \lambda-\rho\right) \gamma_{\varepsilon}^{2}}{1-\lambda}\right]
\end{aligned}
$$

as $(n, T) \stackrel{\text { seq }}{\rightarrow} \infty$.

Note that

$$
\frac{1}{n T} G_{2}=\frac{1}{n T} \mathbf{x}^{\prime} \Phi^{-1} \mathbf{u}-\frac{\mathbf{x}^{\prime} \Phi^{-1} \boldsymbol{\iota}_{n T}}{n}\left(\frac{\boldsymbol{\iota}_{n T}^{\prime} \Phi^{-1} \iota_{n T}}{n}\right)^{-1} \frac{\boldsymbol{\iota}_{n T}^{\prime} \Phi^{-1} \mathbf{u}}{n T}
$$

First consider

$$
\begin{aligned}
& \frac{1}{n T} \mathbf{x}^{\prime} \Phi^{-1} \mathbf{u} \\
= & \frac{1}{\varpi_{e}^{2}} \frac{1}{n} \sum_{i=1}^{n}\left[\frac{1}{T}\left(\frac{\varpi_{e}^{2} \mu_{i} T}{\varpi_{e}^{2}+\theta \sigma_{\mu}^{2}} \frac{\mathbf{x}_{i}^{\prime} \mathbf{A}^{-1} \iota_{T}}{T}\right)+\left(\frac{\mathbf{x}_{i}^{\prime} \mathbf{A}^{-1} \boldsymbol{\nu}_{i}}{T}-\frac{\sigma_{\mu}^{2} T}{\varpi_{e}^{2}+\theta \sigma_{\mu}^{2}} \frac{\mathbf{x}_{i}^{\prime} \mathbf{A}^{-1} \iota_{T}}{T} \frac{\boldsymbol{\iota}_{T}^{\prime} \mathbf{A}^{-1} \boldsymbol{\nu}_{i}}{T}\right)\right] .
\end{aligned}
$$

Because $\frac{1}{T} \mathbf{x}_{i}^{\prime} \mathbf{A}^{-1} \iota_{T} \stackrel{p}{\rightarrow} 0$, which has been proved in part (a), and

$$
\frac{1}{T} \mathbf{x}_{i}^{\prime} \mathbf{A}^{-1} \boldsymbol{\nu}_{i}=\frac{1}{T} \sum_{t=1}^{T}\left(x_{i t}-\rho x_{i t-1}\right) e_{i t} \stackrel{p}{\rightarrow} \lim \frac{1}{T} \sum_{t=1}^{T} E\left[\left(x_{i t}-\rho x_{i t-1}\right) e_{i t}\right],
$$

and

$$
\frac{1}{T} \boldsymbol{\iota}_{T}^{\prime} \mathbf{A}^{-1} \boldsymbol{\nu}_{i}=(1-\rho) \frac{1}{T} \sum_{t=1}^{T} e_{i t} \stackrel{p}{\rightarrow} 0
$$

as $(n, T) \stackrel{\text { seq }}{\rightarrow} \infty$. Hence,

$$
\begin{aligned}
& \frac{1}{n T} \mathbf{x}^{\prime} \Phi^{-1} \mathbf{u} \\
= & \frac{1}{\varpi_{e}^{2}} \frac{1}{n} \sum_{i=1}^{n}\left[\frac{1}{T}\left(\frac{\varpi_{e}^{2} \mu_{i} T}{\varpi_{e}^{2}+\theta \sigma_{\mu}^{2}} \frac{\mathbf{x}_{i}^{\prime} \mathbf{A}^{-1} \iota_{T}}{T}\right)+\left(\frac{\mathbf{x}_{i}^{\prime} \mathbf{A}^{-1} \boldsymbol{\nu}_{i}}{T}-\frac{\sigma_{\mu}^{2} T}{\varpi_{e}^{2}+\theta \sigma_{\mu}^{2}} \frac{\mathbf{x}_{i}^{\prime} \mathbf{A}^{-1} \iota_{T}}{T} \frac{\boldsymbol{\iota}_{T}^{\prime} \mathbf{A}^{-1} \boldsymbol{\nu}_{i}}{T}\right)\right] \\
& \stackrel{p}{\rightarrow} \frac{1}{T} o(1)+\frac{1}{\varpi_{e}^{2}} \lim \frac{1}{n} \sum_{i=1}^{n} \frac{1}{T} \sum_{t=1}^{T} E\left[\left(x_{i t}-\rho x_{i t-1}\right) e_{i t}\right] \\
= & \frac{1}{\varpi_{e}^{2}} \lim \frac{1}{n} \sum_{i=1}^{n} \frac{1}{T} \sum_{t=1}^{T} E\left[\left(x_{i t}-\rho x_{i t-1}\right) e_{i t}\right]
\end{aligned}
$$

as $(n, T) \stackrel{\text { seq }}{\rightarrow} \infty$. 
Also because

$$
\frac{1}{n T} \boldsymbol{\iota}_{n T}^{\prime} \Phi^{-1} \mathbf{u}=\frac{1}{n} \sum_{i=1}^{n} \frac{\theta}{\sigma_{e}^{2}+\theta \sigma_{\mu}^{2}}\left(\frac{\mu_{i}}{T}+\frac{\boldsymbol{\iota}_{T}^{\prime} \mathbf{A}^{-1} \boldsymbol{\nu}_{i}}{T}\right) \stackrel{p}{\rightarrow} 0
$$

and $\frac{1}{n} \mathbf{x}^{\prime} \Phi^{-1} \boldsymbol{\iota}_{n T} \stackrel{p}{\rightarrow} 0$ and $\frac{1}{n} \boldsymbol{\iota}_{n T}^{\prime} \Phi^{-1} \iota_{n T} \stackrel{p}{\rightarrow} \frac{1}{\sigma_{\mu}^{2}}$, which are proved in part (a).

Hence, we have

$$
\begin{aligned}
\frac{1}{n T} G_{2}= & \frac{1}{n T} \mathbf{x}^{\prime} \Phi^{-1} \mathbf{u}-\frac{\mathbf{x}^{\prime} \Phi^{-1} \iota_{n T}}{n}\left(\frac{\boldsymbol{\iota}_{n T}^{\prime} \Phi^{-1} \iota_{n T}}{n}\right)^{-1} \frac{\boldsymbol{\iota}_{n T}^{\prime} \Phi^{-1} \mathbf{u}}{n T} \\
& \stackrel{p}{\rightarrow} \frac{1}{\varpi_{e}^{2}} \lim \frac{1}{n} \sum_{i=1}^{n} \frac{1}{T} \sum_{t=1}^{T} E\left[\left(x_{i t}-\rho x_{i t-1}\right) e_{i t}\right]
\end{aligned}
$$

as $(n, T) \stackrel{\text { seq }}{\rightarrow} \infty$.

Note that

$$
\frac{1}{\sqrt{n T}} G_{2}=\frac{1}{\sqrt{n T}} \mathbf{x}^{\prime} \Phi^{-1} \mathbf{u}-\frac{\mathbf{x}^{\prime} \Phi^{-1} \boldsymbol{\iota}_{n T}}{n}\left(\frac{\boldsymbol{\iota}_{n T}^{\prime} \Phi^{-1} \boldsymbol{\iota}_{n T}}{n}\right)^{-1} \frac{\boldsymbol{\iota}_{N T}^{\prime} \Phi^{-1} \mathbf{u}}{\sqrt{n T}}
$$

First consider

$$
\begin{aligned}
& \sqrt{n T} \frac{1}{n T} \mathbf{x}^{\prime} \Phi^{-1} \mathbf{u} \\
= & \frac{1}{\varpi_{e}^{2}} \frac{1}{\sqrt{n}} \sum_{i=1}^{n}\left[\frac{1}{\sqrt{T}}\left(\frac{\varpi_{e}^{2} \mu_{i} T}{\varpi_{e}^{2}+\theta \sigma_{\mu}^{2}} \frac{\mathbf{x}_{i}^{\prime} \mathbf{A}^{-1} \iota_{T}}{T}\right)+\left(\frac{\mathbf{x}_{i}^{\prime} \mathbf{A}^{-1} \boldsymbol{\nu}_{i}}{\sqrt{T}}-\frac{\sigma_{\mu}^{2} T}{\varpi_{e}^{2}+\theta \sigma_{\mu}^{2}} \frac{\mathbf{x}_{i}^{\prime} \mathbf{A}^{-1} \iota_{T}}{T} \frac{\boldsymbol{\iota}_{T}^{\prime} \mathbf{A}^{-1} \boldsymbol{\nu}_{i}}{\sqrt{T}}\right)\right]
\end{aligned}
$$

For a fixed $n, \frac{1}{T} \mathbf{x}_{i}^{\prime} \mathbf{A}^{-1} \iota_{T} \stackrel{p}{\rightarrow} 0$, which has been proved in part (a), and

$$
\begin{aligned}
& \sqrt{T}\left[\frac{1}{T} \mathbf{x}_{i}^{\prime} \mathbf{A}^{-1} \boldsymbol{\nu}_{i}-\lim \frac{1}{T} \sum_{t=1}^{T} E\left[\left(x_{i t}-\rho x_{i t-1}\right) e_{i t}\right]\right] \\
= & \sqrt{T}\left[\frac{1}{T} \sum_{t=1}^{T}\left(x_{i t}-\rho x_{i t-1}\right) e_{i t}-\lim \frac{1}{T} \sum_{t=1}^{T} E\left[\left(x_{i t}-\rho x_{i t-1}\right) e_{i t}\right]\right] \\
= & \sqrt{T}\left[\frac{1}{T} \sum_{t=1}^{T}\left(\varepsilon_{i t}+(\lambda-\rho) x_{i t-1}\right) e_{i t}-\lim \frac{1}{T} \sum_{t=1}^{T} E\left[\left(x_{i t}-\rho x_{i t-1}\right) e_{i t}\right]\right] \\
= & \sqrt{T}\left\{\frac{1}{T} \sum_{t=1}^{T}\left[\left(\varepsilon_{i t}+(\lambda-\rho) \varepsilon_{i t-1}+\lambda(\lambda-\rho) \varepsilon_{i t-2}+\lambda^{2}(\lambda-\rho) \varepsilon_{i t-3}+\cdots\right) e_{i t}\right]\right. \\
& \left.-\lim \frac{1}{T} \sum_{t=1}^{T} E\left[\left(x_{i t}-\rho x_{i t-1}\right) e_{i t}\right]\right\} \\
= & N\left(0, \frac{\left(1-2 \rho \lambda+\rho^{2}\right) \psi_{00}}{1-\lambda^{2}}\right)
\end{aligned}
$$


where $\psi_{00}=E\left(\varepsilon_{t}^{2} e_{t}^{2}\right)$ and

$$
\frac{1}{\sqrt{T}} \boldsymbol{\iota}_{T}^{\prime} \mathbf{A}^{-1} \boldsymbol{\nu}_{i}=(1-\rho) \frac{1}{\sqrt{T}} \sum_{t=1}^{T} e_{i t} \Rightarrow(1-\rho) \varpi_{e} N(0,1)
$$

as $T \rightarrow \infty$. Hence,

$$
\begin{aligned}
& \sqrt{n T}\left[\frac{1}{n T} \mathbf{x}^{\prime} \Phi^{-1} \mathbf{u}-\frac{1}{\varpi_{e}^{2}} \lim \frac{1}{n} \sum_{i=1}^{n} \frac{1}{T} \sum_{t=1}^{T} E\left[\left(x_{i t}-\rho x_{i t-1}\right) e_{i t}\right]\right] \\
&= \frac{1}{\varpi_{e}^{2}} \frac{1}{\sqrt{n}} \sum_{i=1}^{n}\left[\frac{1}{\sqrt{T}}\left(\frac{\varpi_{e}^{2} \mu_{i} T}{\varpi_{e}^{2}+\theta \sigma_{\mu}^{2}} \frac{\mathbf{x}_{i}^{\prime} \mathbf{A}^{-1} \iota_{T}}{T}\right)+\left(\frac{\mathbf{x}_{i}^{\prime} \mathbf{A}^{-1} \boldsymbol{\nu}_{i}}{\sqrt{T}}-\frac{\sigma_{\mu}^{2} T}{\varpi_{e}^{2}+\theta \sigma_{\mu}^{2}} \frac{\mathbf{x}_{i}^{\prime} \mathbf{A}^{-1} \boldsymbol{\iota}_{T}}{T} \frac{\boldsymbol{\iota}_{T}^{\prime} \mathbf{A}^{-1} \boldsymbol{\nu}_{i}}{\sqrt{T}}\right)\right] \\
&-\frac{1}{\varpi_{e}^{2}} \sqrt{n T} \lim \frac{1}{n} \sum_{i=1}^{n} \frac{1}{T} \sum_{t=1}^{T} E\left[\left(x_{i t}-\rho x_{i t-1}\right) e_{i t}\right] \\
& \Rightarrow \frac{1}{\varpi_{e}^{2}} \frac{1}{\sqrt{n}} \sum_{i=1}^{n} N\left(0, \frac{\left(1-2 \rho \lambda+\rho^{2}\right) \psi_{00}}{1-\lambda^{2}}\right) \\
&= \frac{1}{\varpi_{e}^{2}} N\left(0, \frac{\left(1-2 \rho \lambda+\rho^{2}\right) \psi_{00}}{1-\lambda^{2}}\right) \\
& \text { as }(n, T) \stackrel{\text { seq }}{\rightarrow} \infty .
\end{aligned}
$$

Also consider that

$$
\begin{aligned}
\frac{1}{\sqrt{n T}} \boldsymbol{\iota}_{n T}^{\prime} \Phi^{-1} \mathbf{u} & =\frac{1}{\sqrt{n}} \sum_{i=1}^{n} \frac{1}{\sqrt{T}} \frac{\theta}{\sigma_{e}^{2}+\theta \sigma_{\mu}^{2}}\left(\mu_{i}+\boldsymbol{\iota}_{T}^{\prime} \mathbf{A}^{-1} \boldsymbol{\nu}_{i}\right) \\
& =\frac{1}{\sqrt{T}} \frac{1}{\sqrt{n}} \sum_{i=1}^{n} \frac{\theta}{\sigma_{e}^{2}+\theta \sigma_{\mu}^{2}} \mu_{i}+\frac{1}{\sqrt{n}} \sum_{i=1}^{n} \frac{\theta}{\sigma_{e}^{2}+\theta \sigma_{\mu}^{2}} \frac{\boldsymbol{\iota}_{T}^{\prime} \mathbf{A}^{-1} \boldsymbol{\nu}_{i}}{\sqrt{T}}
\end{aligned}
$$

It is easy to see that

$$
\frac{1}{\sqrt{T}} \frac{1}{\sqrt{n}} \sum_{i=1}^{n} \frac{\theta}{\sigma_{e}^{2}+\theta \sigma_{\mu}^{2}} \mu_{i}=\frac{1}{\sqrt{\bar{T}}} o_{p}(1)
$$

Also, for a fixed $n$,

$$
\frac{1}{\sqrt{n}} \sum_{i=1}^{n} \frac{\theta}{\sigma_{e}^{2}+\theta \sigma_{\mu}^{2}} \frac{\iota_{T}^{\prime} \mathbf{A}^{-1} \boldsymbol{\nu}_{i}}{\sqrt{T}} \Rightarrow \frac{1}{\sqrt{n}} \sum_{i=1}^{n}\left[\frac{(1-\rho) \varpi_{e}}{\sigma_{\mu}^{2}} N(0,1)\right]
$$

as $T \rightarrow \infty$ because $\frac{1}{\sqrt{T}} \boldsymbol{\iota}_{T}^{\prime} \mathbf{A}^{-1} \boldsymbol{\nu}_{i} \Rightarrow(1-\rho) \varpi_{e} N(0,1)$, which has been proved in part (e). Also,

$$
\frac{1}{\sqrt{n}} \sum_{i=1}^{n}\left[\frac{(1-\rho) \varpi_{e}}{\sigma_{\mu}^{2}} N(0,1)\right] \Rightarrow \frac{(1-\rho) \varpi_{e}}{\sigma_{\mu}^{2}} N(0,1)
$$


as $n \rightarrow \infty$. Therefore,

$$
\frac{1}{\sqrt{n}} \sum_{i=1}^{n} \frac{\theta}{\sigma_{e}^{2}+\theta \sigma_{\mu}^{2}} \frac{\boldsymbol{\iota}_{T}^{\prime} \mathbf{A}^{-1} \boldsymbol{\nu}_{i}}{\sqrt{T}} \Rightarrow \frac{(1-\rho) \varpi_{e}}{\sigma_{\mu}^{2}} N(0,1)
$$

as $(n, T) \stackrel{\text { seq }}{\longrightarrow} \infty$. So,

$$
\frac{1}{\sqrt{n T}} \boldsymbol{\iota}_{n T}^{\prime} \Phi^{-1} \mathbf{u} \Rightarrow \frac{(1-\rho) \varpi_{e}}{\sigma_{\mu}^{2}} N(0,1)
$$

Hence, we have

$$
\begin{aligned}
& \frac{1}{\sqrt{n T}} G_{2}-\sqrt{n T} \frac{1}{\varpi_{e}^{2}} \lim \frac{1}{n} \sum_{i=1}^{n} \frac{1}{T} \sum_{t=1}^{T} E\left[\left(x_{i t}-\rho x_{i t-1}\right) e_{i t}\right] \\
= & \frac{1}{\sqrt{n T}} \mathbf{x}^{\prime} \Phi^{-1} \mathbf{u}-\sqrt{n T} \frac{1}{\varpi_{e}^{2}} \lim \frac{1}{n} \sum_{i=1}^{n} \frac{1}{T} \sum_{t=1}^{T} E\left[\left(x_{i t}-\rho x_{i t-1}\right) e_{i t}\right] \\
& -\frac{\mathbf{x}^{\prime} \Phi^{-1} \iota_{n T}}{n}\left(\frac{\boldsymbol{\iota}_{n T}^{\prime} \Phi^{-1} \iota_{n T}}{n}\right)^{-1} \frac{\boldsymbol{\iota}_{N T}^{\prime} \Phi^{-1} \mathbf{u}}{\sqrt{n T}} \\
\Rightarrow & \frac{1}{\sigma_{e}^{2}} N\left(0, \frac{\left(1-2 \rho \lambda+\rho^{2}\right) \psi_{00}}{1-\lambda^{2}}\right) .
\end{aligned}
$$

Note that

$$
\frac{1}{n T} G_{1}=\frac{1}{n T} \mathbf{x}^{\prime} \Phi^{-1} \mathbf{x}-\frac{1}{T} \frac{\mathbf{x}^{\prime} \Phi^{-1} \boldsymbol{\iota}_{n T}}{n}\left(\frac{\boldsymbol{\iota}_{n T}^{\prime} \Phi^{-1} \iota_{n T}}{n}\right)^{-1} \frac{\boldsymbol{\iota}_{n T}^{\prime} \Phi^{-1} \mathbf{x}}{n}
$$

First consider that

$$
\begin{aligned}
& \frac{1}{n T} \mathbf{x}^{\prime} \Phi^{-1} \mathbf{x} \\
= & \frac{1}{\varpi_{e}^{2}} \frac{1}{n} \sum_{i=1}^{n}\left(\frac{\mathbf{x}_{i}^{\prime} \mathbf{A}^{-1} \mathbf{x}_{i}}{T}-\frac{\sigma_{\mu}^{2}}{\varpi_{e}^{2}+\theta \sigma_{\mu}^{2}} \frac{\mathbf{x}_{i}^{\prime} \mathbf{A}^{-1} \iota_{T}}{\sqrt{T}} \frac{\boldsymbol{\iota}_{T}^{\prime} \mathbf{A}^{-1} \mathbf{x}_{i}}{\sqrt{T}}\right)
\end{aligned}
$$

for a fixed $n$,

$$
\begin{aligned}
& \frac{1}{T} \mathbf{x}_{i}^{\prime} \mathbf{A}^{-1} \mathbf{x}_{i} \\
= & \frac{1}{T} \sum_{t=1}^{T}\left(x_{i t}-x_{i t-1}\right)^{2} \\
= & \frac{1}{T} \sum_{t=1}^{T} x_{i t}^{2}+\frac{1}{T} \sum_{t=1}^{T} x_{i t-1}^{2}-\frac{1}{T} \sum_{t=1}^{T} 2 x_{i t-1} x_{i t} \\
& \frac{p}{\rightarrow} 2\left(\frac{\sigma_{\varepsilon}^{2}}{1-\lambda^{2}}+\frac{2 \lambda \gamma_{\varepsilon}^{2}}{1-\lambda}\right)-2\left[\lambda\left(\frac{\sigma_{\varepsilon}^{2}}{1-\lambda^{2}}+\frac{2 \lambda \gamma_{\varepsilon}^{2}}{1-\lambda}\right)+\frac{\gamma_{\varepsilon}^{2}}{1-\lambda}\right] \\
= & \frac{2 \sigma_{\varepsilon}^{2}}{1+\lambda}+\frac{2\left(-2 \lambda^{2}+2 \lambda-1\right) \gamma_{\varepsilon}^{2}}{1-\lambda}
\end{aligned}
$$


and

$$
\frac{1}{\sqrt{T}} \mathbf{x}_{i}^{\prime} \mathbf{A}^{-1} \iota_{T}=\frac{1}{\sqrt{T}} x_{i 1} \stackrel{p}{\rightarrow} 0
$$

as $T \rightarrow \infty$. Hence

$$
\begin{aligned}
& \frac{1}{n T} \mathbf{x}^{\prime} \Phi^{-1} \mathbf{x} \\
= & \frac{1}{\varpi_{e}^{2}} \frac{1}{n} \sum_{i=1}^{n}\left(\frac{\mathbf{x}_{i}^{\prime} \mathbf{A}^{-1} \mathbf{x}_{i}}{T}-\frac{\sigma_{\mu}^{2}}{\varpi_{e}^{2}+\theta \sigma_{\mu}^{2}} \frac{\mathbf{x}_{i}^{\prime} \mathbf{A}^{-1} \iota_{T}}{\sqrt{T}} \frac{\boldsymbol{\iota}_{T}^{\prime} \mathbf{A}^{-1} \mathbf{x}_{i}}{\sqrt{T}}\right) \\
& \stackrel{p}{\rightarrow} \frac{1}{\varpi_{e}^{2}} \frac{1}{n} \sum_{i=1}^{n}\left[\frac{2 \sigma_{\varepsilon}^{2}}{1+\lambda}+\frac{2\left(-2 \lambda^{2}+2 \lambda-1\right) \gamma_{\varepsilon}^{2}}{1-\lambda}\right] \\
= & \frac{1}{\varpi_{e}^{2}}\left[\frac{2 \sigma_{\varepsilon}^{2}}{1+\lambda}+\frac{2\left(-2 \lambda^{2}+2 \lambda-1\right) \gamma_{\varepsilon}^{2}}{1-\lambda}\right]
\end{aligned}
$$

holds for all $n$ and hence it holds for a large $n$ as well.

Also because that

$$
\frac{1}{n} \mathbf{x}^{\prime} \Phi^{-1} \iota_{n T}=\frac{1}{n} \sum_{i=1}^{n} \frac{1}{\sigma_{e}^{2}+\theta \sigma_{\mu}^{2}}\left(X_{i}^{\prime} A^{-1} \iota_{T}\right) \stackrel{p}{\rightarrow} 0
$$

since $\theta=1$, and

$$
\frac{1}{n} \iota_{N T}^{\prime} \Phi^{-1} \iota_{n T}=\frac{1}{n} \frac{n \theta}{\varpi_{e}^{2}+\theta \sigma_{\mu}^{2}} \stackrel{p}{\rightarrow} \frac{1}{\sigma_{\mu}^{2}}
$$

Hence, we have

$$
\begin{aligned}
\frac{1}{n T} G_{1}= & \frac{1}{n T} \mathbf{x}^{\prime} \Phi^{-1} \mathbf{x}-\frac{1}{T} \frac{\mathbf{x}^{\prime} \Phi^{-1} \boldsymbol{\iota}_{n T}}{n}\left(\frac{\boldsymbol{\iota}_{n T}^{\prime} \Phi^{-1} \boldsymbol{\iota}_{n T}}{n}\right)^{-1} \frac{\boldsymbol{\iota}_{n T}^{\prime} \Phi^{-1} \mathbf{x}}{n} \\
& \stackrel{p}{\rightarrow} \frac{1}{\varpi_{e}^{2}}\left[\frac{2 \sigma_{\varepsilon}^{2}}{1+\lambda}+\frac{2\left(-2 \lambda^{2}+2 \lambda-1\right) \gamma_{\varepsilon}^{2}}{1-\lambda}\right]
\end{aligned}
$$

as $(n, T) \stackrel{\text { seq }}{\rightarrow} \infty$.

Note that

$$
\frac{1}{n T} G_{2}=\frac{1}{n T} \mathbf{x}^{\prime} \Phi^{-1} \mathbf{u}-\frac{\mathbf{x}^{\prime} \Phi^{-1} \iota_{n T}}{n}\left(\frac{\boldsymbol{\iota}_{n T}^{\prime} \Phi^{-1} \iota_{n T}}{n}\right)^{-1} \frac{\boldsymbol{\iota}_{n T}^{\prime} \Phi^{-1} \mathbf{u}}{n T}
$$

First consider that

$$
\begin{aligned}
& \frac{1}{n T} \mathbf{x}^{\prime} \Phi^{-1} \mathbf{u} \\
= & \frac{1}{\varpi_{e}^{2}} \frac{1}{n} \sum_{i=1}^{n}\left[\frac{1}{\sqrt{T}}\left(\frac{\varpi_{e}^{2} \mu_{i}}{\varpi_{e}^{2}+\theta \sigma_{\mu}^{2}} \frac{\mathbf{x}_{i}^{\prime} \mathbf{A}^{-1} \iota_{T}}{\sqrt{T}}\right)+\left(\frac{\mathbf{x}_{i}^{\prime} \mathbf{A}^{-1} \boldsymbol{\nu}_{i}}{T}-\frac{\sigma_{\mu}^{2}}{\varpi_{e}^{2}+\theta \sigma_{\mu}^{2}} \frac{\mathbf{x}_{i}^{\prime} \mathbf{A}^{-1} \iota_{T}}{\sqrt{T}} \frac{\boldsymbol{\iota}_{T}^{\prime} \mathbf{A}^{-1} \boldsymbol{\nu}_{i}}{\sqrt{T}}\right)\right]
\end{aligned}
$$


For a fixed $n$, because $\frac{1}{\sqrt{T}} \mathbf{x}_{i}^{\prime} \mathbf{A}^{-1} \iota_{T} \stackrel{p}{\rightarrow} 0$ which is proved in part (a), and

$$
\frac{1}{T} \mathbf{x}_{i}^{\prime} \mathbf{A}^{-1} \boldsymbol{\nu}_{i}=\frac{1}{T} \sum_{t=1}^{T}\left(x_{i t}-x_{i t-1}\right) e_{i t} \stackrel{p}{\rightarrow} \lim \frac{1}{T} \sum_{t=1}^{T} E\left[\left(x_{i t}-x_{i t-1}\right) e_{i t}\right],
$$

and

$$
\frac{1}{\sqrt{T}} \boldsymbol{\iota}_{T}^{\prime} \mathbf{A}^{-1} \boldsymbol{\nu}_{i}=\frac{1}{\sqrt{T}} \nu_{i 1}^{\prime} \stackrel{p}{\rightarrow} 0
$$

as $T \rightarrow \infty$. Hence,

$$
\begin{aligned}
& \frac{1}{n T} \mathbf{x}^{\prime} \Phi^{-1} \mathbf{u} \\
= & \frac{1}{\varpi_{e}^{2}} \frac{1}{n} \sum_{i=1}^{n}\left[\frac{1}{\sqrt{T}}\left(\frac{\varpi_{e}^{2} \mu_{i}}{\varpi_{e}^{2}+\theta \sigma_{\mu}^{2}} \frac{\mathbf{x}_{i}^{\prime} \mathbf{A}^{-1} \iota_{T}}{\sqrt{T}}\right)+\left(\frac{\mathbf{x}_{i}^{\prime} \mathbf{A}^{-1} \boldsymbol{\nu}_{i}}{T}-\frac{\sigma_{\mu}^{2}}{\varpi_{e}^{2}+\theta \sigma_{\mu}^{2}} \frac{\mathbf{x}_{i}^{\prime} \mathbf{A}^{-1} \iota_{T}}{\sqrt{T}} \frac{\boldsymbol{\iota}_{T}^{\prime} \mathbf{A}^{-1} \boldsymbol{\nu}_{i}}{\sqrt{T}}\right)\right] \\
& \stackrel{p}{\rightarrow} \frac{1}{\varpi_{e}^{2}} \lim \frac{1}{n} \sum_{i=1}^{n} \frac{1}{T} \sum_{t=1}^{T} E\left[\left(x_{i t}-x_{i t-1}\right) e_{i t}\right]
\end{aligned}
$$

as $n \rightarrow \infty$.

Also because that

$$
\begin{aligned}
& \frac{1}{n} \iota_{n T}^{\prime} \Phi^{-1} \mathbf{u} \\
= & \frac{1}{n} \sum_{i=1}^{n} \frac{\theta}{\varpi_{e}^{2}+\theta \sigma_{\mu}^{2}}\left(\mu_{i}+\iota_{T}^{\prime} A^{-1} \nu_{i}\right) \\
= & \frac{1}{n} \sum_{i=1}^{n} \frac{1}{\varpi_{e}^{2}+\sigma_{\mu}^{2}}\left(\mu_{i}+\nu_{i 1}^{\prime}\right) \\
& \stackrel{p}{\rightarrow} 0
\end{aligned}
$$

and $\frac{1}{n} \mathbf{x}^{\prime} \Phi^{-1} \iota_{n T} \stackrel{p}{\rightarrow} 0$ and $\frac{1}{n} \iota_{N T}^{\prime} \Phi^{-1} \iota_{n T} \stackrel{p}{\rightarrow} \frac{1}{\sigma_{\mu}^{2}}$, which are proved in part (a).

Hence, we have

$$
\begin{aligned}
\frac{1}{n T} G_{2}= & \frac{1}{n T} \mathbf{x}^{\prime} \Phi^{-1} \mathbf{u}-\frac{\mathbf{x}^{\prime} \Phi^{-1} \iota_{n T}}{n}\left(\frac{\boldsymbol{\iota}_{n T}^{\prime} \Phi^{-1} \boldsymbol{\iota}_{n T}}{n}\right)^{-1} \frac{\boldsymbol{\iota}_{n T}^{\prime} \Phi^{-1} \mathbf{u}}{n T} \\
& \stackrel{p}{\rightarrow} \frac{1}{\varpi_{e}^{2}} \lim \frac{1}{n} \sum_{i=1}^{n} \frac{1}{T} \sum_{t=1}^{T} E\left[\left(x_{i t}-x_{i t-1}\right) e_{i t}\right]
\end{aligned}
$$

as $(n, T) \stackrel{\text { seq }}{\rightarrow} \infty$.

Note that

$$
\frac{1}{n T} G_{2}=\frac{1}{n T} \mathbf{x}^{\prime} \Phi^{-1} \mathbf{u}-\frac{\mathbf{x}^{\prime} \Phi^{-1} \iota_{n T}}{n}\left(\frac{\boldsymbol{\iota}_{n T}^{\prime} \Phi^{-1} \iota_{n T}}{n}\right)^{-1} \frac{\boldsymbol{\iota}_{n T}^{\prime} \Phi^{-1} \mathbf{u}}{n T}
$$


First consider that

$$
\begin{aligned}
& \sqrt{n T} \frac{1}{n T} \mathbf{x}^{\prime} \Phi^{-1} \mathbf{u} \\
= & \frac{1}{\varpi_{e}^{2}} \frac{1}{\sqrt{n}} \sum_{i=1}^{n}\left[\left(\frac{\varpi_{e}^{2} \mu_{i}}{\varpi_{e}^{2}+\theta \sigma_{\mu}^{2}} \frac{\mathbf{x}_{i}^{\prime} \mathbf{A}^{-1} \iota_{T}}{\sqrt{T}}\right)+\left(\frac{\mathbf{x}_{i}^{\prime} \mathbf{A}^{-1} \boldsymbol{\nu}_{i}}{\sqrt{T}}-\frac{\sigma_{\mu}^{2}}{\varpi_{e}^{2}+\theta \sigma_{\mu}^{2}} \frac{1}{\sqrt{T}} \mathbf{x}_{i}^{\prime} \mathbf{A}^{-1} \iota_{T} \boldsymbol{\iota}_{T}^{\prime} \mathbf{A}^{-1} \boldsymbol{\nu}_{i}\right)\right]
\end{aligned}
$$

For a fixed $n$, because $\frac{1}{\sqrt{T}} \mathbf{x}_{i}^{\prime} \mathbf{A}^{-1} \iota_{T} \stackrel{p}{\rightarrow} 0$ which is proved in part (a), and

$$
\begin{aligned}
& \sqrt{T}\left(\frac{1}{T} \mathbf{x}_{i}^{\prime} \mathbf{A}^{-1} \boldsymbol{\nu}_{i}-\lim \frac{1}{T} \sum_{t=1}^{T} E\left[\left(x_{i t}-x_{i t-1}\right) e_{i t}\right]\right) \\
= & \frac{1}{\sqrt{T}} \sum_{t=1}^{T}\left(x_{i t}-x_{i t-1}\right) e_{i t}-\sqrt{T} \lim \frac{1}{T} \sum_{t=1}^{T} E\left[\left(x_{i t}-x_{i t-1}\right) e_{i t}\right] \\
= & \frac{1}{\sqrt{T}} \sum_{t=1}^{T}\left(\varepsilon_{i t}+(\lambda-1) x_{i t-1}\right) e_{i t}-\sqrt{T} \lim \frac{1}{T} \sum_{t=1}^{T} E\left[\left(x_{i t}-x_{i t-1}\right) e_{i t}\right] \\
= & \frac{1}{\sqrt{T}} \sum_{t=1}^{T}\left[\left(\varepsilon_{i t}+(\lambda-1) \varepsilon_{i t-1}+\lambda(\lambda-1) \varepsilon_{i t-2}+\lambda^{2}(\lambda-1) \varepsilon_{i t-3}+\cdots\right) e_{i t}\right] \\
& -\sqrt{T} \lim \frac{1}{T} \sum_{t=1}^{T} E\left[\left(x_{i t}-x_{i t-1}\right) e_{i t}\right] \\
\Rightarrow & N\left(0, \frac{2 \psi_{00}}{1+\lambda}\right)
\end{aligned}
$$

where $\psi_{00}=E\left(\varepsilon_{t}^{2} e_{t}^{2}\right)$, and

$$
\frac{1}{\sqrt{T}} \mathbf{x}_{i}^{\prime} \mathbf{A}^{-1} \iota_{T} \iota_{T}^{\prime} \mathbf{A}^{-1} \boldsymbol{\nu}_{i}=\frac{1}{\sqrt{T}} x_{i 1} \nu_{i 1}^{\prime} \stackrel{p}{\rightarrow} 0,
$$


as $T \rightarrow \infty$. Hence,

$$
\begin{aligned}
& \sqrt{n T}\left(\frac{1}{n T} \mathbf{x}^{\prime} \Phi^{-1} \mathbf{u}-\frac{1}{\varpi_{e}^{2}} \lim \frac{1}{n} \sum_{i=1}^{n} \frac{1}{T} \sum_{t=1}^{T} E\left[\left(x_{i t}-x_{i t-1}\right) e_{i t}\right]\right) \\
= & \frac{1}{\varpi_{e}^{2}} \frac{1}{\sqrt{n}} \sum_{i=1}^{n}\left(\frac{\varpi_{e}^{2} \mu_{i}}{\varpi_{e}^{2}+\theta \sigma_{\mu}^{2}} \frac{\mathbf{x}_{i}^{\prime} \mathbf{A}^{-1} \boldsymbol{\iota}_{T}}{\sqrt{T}}\right) \\
& +\frac{1}{\varpi_{e}^{2}} \frac{1}{\sqrt{n}} \sum_{i=1}^{n}\left(\frac{\mathbf{x}_{i}^{\prime} \mathbf{A}^{-1} \boldsymbol{\nu}_{i}}{\sqrt{T}}-\frac{\sigma_{\mu}^{2}}{\varpi_{e}^{2}+\theta \sigma_{\mu}^{2}} \frac{1}{\sqrt{T}} \mathbf{x}_{i}^{\prime} \mathbf{A}^{-1} \iota_{T} \boldsymbol{\iota}_{T}^{\prime} \mathbf{A}^{-1} \boldsymbol{\nu}_{i}\right) \\
& -\sqrt{n T} \frac{1}{\varpi_{e}^{2}} \lim \frac{1}{n} \sum_{i=1}^{n} \frac{1}{T} \sum_{t=1}^{T} E\left[\left(x_{i t}-x_{i t-1}\right) e_{i t}\right] \\
\Rightarrow & o_{p}(1)+\frac{1}{\varpi_{e}^{2}} \frac{1}{\sqrt{n}} \sum_{i=1}^{n}\left[N\left(0, \frac{2 \psi_{00}}{1+\lambda}\right)-o_{p}(1)\right] \\
\Rightarrow & \frac{1}{\varpi_{e}^{2}} N\left(0, \frac{2 \psi_{00}}{1+\lambda}\right)
\end{aligned}
$$

as $(n, T) \stackrel{\text { seq }}{\rightarrow} \infty$.

Also because that

$$
\begin{aligned}
& \frac{1}{\sqrt{n}} \iota_{n T}^{\prime} \Phi^{-1} \mathbf{u} \\
= & \frac{1}{\sqrt{n}} \sum_{i=1}^{n} \frac{\theta}{\varpi_{e}^{2}+\theta \sigma_{\mu}^{2}}\left(\mu_{i}+\iota_{T}^{\prime} A^{-1} \nu_{i}\right) \\
\Rightarrow & \frac{1}{\sqrt{n}} \sum_{i=1}^{n} \frac{1}{\varpi_{e}^{2}+\sigma_{\mu}^{2}}\left(\mu_{i}+\nu_{i 1}^{\prime}\right) \\
\Rightarrow & \frac{1}{\varpi_{e}^{2}+\sigma_{\mu}^{2}} N(0,1)
\end{aligned}
$$

and also because $\frac{1}{n} \mathbf{x}^{\prime} \Phi^{-1} \iota_{n T} \stackrel{p}{\rightarrow} 0$ and $\frac{1}{n} \iota_{N T}^{\prime} \Phi^{-1} \iota_{n T} \stackrel{p}{\rightarrow} \frac{1}{\sigma_{\mu}^{2}}$, which are proved in part (a). Hence, we have

$$
\begin{aligned}
& \frac{1}{\sqrt{n T}} G_{2}-\sqrt{n T} \frac{1}{\varpi_{e}^{2}} \lim \frac{1}{n} \sum_{i=1}^{n} \frac{1}{T} \sum_{t=1}^{T} E\left[\left(x_{i t}-x_{i t-1}\right) e_{i t}\right] \\
= & \frac{1}{\sqrt{n T}} \mathbf{x}^{\prime} \Phi^{-1} \mathbf{u}-\sqrt{n T} \frac{1}{\varpi_{e}^{2}} \lim \frac{1}{n} \sum_{i=1}^{n} \frac{1}{T} \sum_{t=1}^{T} E\left[\left(x_{i t}-\rho x_{i t-1}\right) e_{i t}\right] \\
& -\frac{\mathbf{x}^{\prime} \Phi^{-1} \iota_{n T}}{n}\left(\frac{\boldsymbol{\iota}_{n T}^{\prime} \Phi^{-1} \boldsymbol{\iota}_{n T}}{n}\right)^{-1} \frac{\boldsymbol{\iota}_{n T}^{\prime} \Phi^{-1} \mathbf{u}}{\sqrt{n T}} \\
\Rightarrow & \frac{1}{\varpi_{e}^{2}} N\left(0, \frac{2 \psi_{00}}{1+\lambda}\right) .
\end{aligned}
$$


Note that

$$
\frac{1}{n T^{2}} G_{1}=\frac{1}{n T^{2}} \mathbf{x}^{\prime} \Phi^{-1} \mathbf{x}-\frac{1}{T} \frac{\mathbf{x}^{\prime} \Phi^{-1} \boldsymbol{\iota}_{n T}}{n \sqrt{T}}\left(\frac{\boldsymbol{\iota}_{n T}^{\prime} \Phi^{-1} \boldsymbol{\iota}_{n T}}{n}\right)^{-1} \frac{\boldsymbol{\iota}_{n T}^{\prime} \Phi^{-1} \mathbf{x}}{n \sqrt{T}}
$$

First consider that

$$
\frac{1}{n T^{2}} \mathbf{x}^{\prime} \Phi^{-1} \mathbf{x}=\frac{1}{\varpi_{e}^{2}} \frac{1}{n} \sum_{i=1}^{n}\left(\frac{1}{T^{2}} \mathbf{x}_{i}^{\prime} \mathbf{A}^{-1} \mathbf{x}_{i}-\frac{T \sigma_{\mu}^{2}}{\varpi_{e}^{2}+\theta \sigma_{\mu}^{2}} \frac{\mathbf{x}_{i}^{\prime} \mathbf{A}^{-1} \iota_{T}}{T \sqrt{T}} \frac{\boldsymbol{\iota}_{T}^{\prime} \mathbf{A}^{-1} \mathbf{x}_{i}}{T \sqrt{T}}\right)
$$

For a fixed $n$, because $\theta=O\left((1-\rho)^{2} T\right)$ when $|\rho|<1$ and

$$
\begin{aligned}
& \frac{1}{T^{2}} \mathbf{x}_{i}^{\prime} \mathbf{A}^{-1} \mathbf{x}_{i} \\
= & \frac{1}{T^{2}} \sum_{t=1}^{T}\left(x_{i t}-\rho x_{i t-1}\right)^{2} \\
= & \frac{1}{T^{2}} \sum_{t=1}^{T}\left((1-\rho) x_{i t-1}+\varepsilon_{i t}\right)^{2} \\
= & (1-\rho)^{2} \frac{1}{T^{2}} \sum_{t=1}^{T} x_{i t-1}^{2}+\frac{1}{T}\left[(1-\rho) \frac{1}{T} \sum_{t=1}^{T} 2 x_{i t-1} \varepsilon_{i t}+\frac{1}{T} \sum_{t=1}^{T} \varepsilon_{i t}^{2}\right] \\
\Rightarrow & (1-\rho)^{2} \varpi_{\varepsilon}^{2} \int W_{i}^{2}+\frac{1}{T} o_{p}(1),
\end{aligned}
$$

and

$$
\begin{aligned}
& \frac{1}{T \sqrt{T}} \mathbf{x}_{i}^{\prime} \mathbf{A}^{-1} \iota_{T} \\
= & (1-\rho) \frac{1}{T \sqrt{T}} \sum_{t=1}^{T}\left(x_{i t}-\rho x_{i t-1}\right) \\
= & (1-\rho) \frac{1}{T \sqrt{T}} \sum_{t=1}^{T}\left((1-\rho) x_{i t-1}+\varepsilon_{i t}\right) \\
= & (1-\rho)^{2} \frac{1}{T \sqrt{T}} \sum_{t=1}^{T} x_{i t-1}+\frac{1}{\sqrt{T}}\left[(1-\rho) \frac{1}{T} \sum_{t=1}^{T} \varepsilon_{i t}\right] \\
\Rightarrow & (1-\rho)^{2} \varpi_{\varepsilon} \int W_{i}+\frac{1}{\sqrt{T}} o_{p}(1),
\end{aligned}
$$


as $T \rightarrow \infty$. Hence

$$
\begin{aligned}
\frac{1}{n T^{2}} \mathbf{x}^{\prime} \Phi^{-1} \mathbf{x}= & \frac{1}{\varpi_{e}^{2}} \frac{1}{n} \sum_{i=1}^{n}\left(\frac{1}{T^{2}} \mathbf{x}_{i}^{\prime} \mathbf{A}^{-1} \mathbf{x}_{i}-\frac{T \sigma_{\mu}^{2}}{\varpi_{e}^{2}+\theta \sigma_{\mu}^{2}} \frac{\mathbf{x}_{i}^{\prime} \mathbf{A}^{-1} \iota_{T}}{T \sqrt{T}} \frac{\iota_{T}^{\prime} \mathbf{A}^{-1} \mathbf{x}_{i}}{T \sqrt{T}}\right) \\
& \stackrel{p}{\rightarrow} \frac{1}{\varpi_{e}^{2}} E\left[(1-\rho)^{2} \varpi_{\varepsilon}^{2} \int W_{i}^{2}-(1-\rho)^{2} \varpi_{\varepsilon}^{2}\left(\int W_{i}\right)^{2}\right] \\
= & \frac{(1-\rho)^{2} \varpi_{\varepsilon}^{2}}{6 \varpi_{e}^{2}}
\end{aligned}
$$

as $n \rightarrow \infty$.

And because

$$
\begin{aligned}
\frac{1}{n \sqrt{T}} \mathbf{x}^{\prime} \Phi^{-1} \iota_{n T}= & \frac{1}{n} \sum_{i=1}^{n} \frac{T}{\varpi_{e}^{2}+\theta \sigma_{\mu}^{2}}\left(\frac{\mathbf{x}_{i}^{\prime} \mathbf{A}^{-1} \iota_{T}}{T \sqrt{T}}\right) \\
\Rightarrow & \frac{1}{n} \sum_{i=1}^{n}\left[\frac{1}{\sigma_{\mu}^{2}}(1-\rho)^{2} \varpi_{\varepsilon} \int W_{i}\right] \\
& \stackrel{p}{\rightarrow} 0
\end{aligned}
$$

because $\theta=O(T)$ and $\frac{1}{T \sqrt{T}} \mathbf{x}_{i}^{\prime} \mathbf{A}^{-1} \iota_{T} \Rightarrow(1-\rho)^{2} \varpi_{\varepsilon} \int W_{i}+o_{p}(1)$, which is proved in (a). And because

$$
\frac{1}{n} \iota_{n T}^{\prime} \Phi^{-1} \iota_{n T}=\frac{1}{n} \frac{n \theta}{\varpi_{e}^{2}+\theta \sigma_{\mu}^{2}} \stackrel{p}{\rightarrow} \frac{1}{\sigma_{\mu}^{2}}
$$

Hence, we have

$$
\begin{aligned}
\frac{1}{n T^{2}} G_{1}= & \frac{1}{n T^{2}} \mathbf{x}^{\prime} \Phi^{-1} \mathbf{x}-\frac{1}{T} \frac{\mathbf{x}^{\prime} \Phi^{-1} \iota_{n T}}{n \sqrt{T}}\left(\frac{\iota_{n T}^{\prime} \Phi^{-1} \iota_{n T}}{n}\right)^{-1} \frac{\boldsymbol{\iota}_{n T}^{\prime} \Phi^{-1} \mathbf{x}}{n \sqrt{T}} \\
& \stackrel{p}{\rightarrow} \frac{(1-\rho)^{2} \varpi_{\varepsilon}^{2}}{6 \varpi_{e}^{2}}
\end{aligned}
$$

Note that

$$
\frac{1}{n T} G_{2}=\frac{1}{n T} \mathbf{x}^{\prime} \Phi^{-1} \mathbf{u}-\frac{\mathbf{x}^{\prime} \Phi^{-1} \boldsymbol{\iota}_{n T}}{n \sqrt{T}}\left(\frac{\boldsymbol{\iota}_{n T}^{\prime} \Phi^{-1} \iota_{n T}}{n}\right)^{-1} \frac{\boldsymbol{\iota}_{n T}^{\prime} \Phi^{-1} \mathbf{u}}{n \sqrt{T}}
$$

First consider that

$$
\begin{aligned}
& \frac{1}{n T} \mathbf{x}^{\prime} \Phi^{-1} \mathbf{u} \\
= & \frac{1}{\varpi_{e}^{2}} \frac{1}{n} \sum_{i=1}^{n}\left[\frac{1}{\sqrt{T}}\left(\frac{\varpi_{e}^{2} \mu_{i} T}{\varpi_{e}^{2}+\theta \sigma_{\mu}^{2}} \frac{\mathbf{x}_{i}^{\prime} \mathbf{A}^{-1} \iota_{T}}{T \sqrt{T}}\right)+\left(\frac{\mathbf{x}_{i}^{\prime} \mathbf{A}^{-1} \boldsymbol{\nu}_{i}}{T}-\frac{\sigma_{\mu}^{2} T}{\varpi_{e}^{2}+\theta \sigma_{\mu}^{2}} \frac{\mathbf{x}_{i}^{\prime} \mathbf{A}^{-1} \iota_{T}}{T \sqrt{T}} \frac{\boldsymbol{\iota}_{T}^{\prime} \mathbf{A}^{-1} \boldsymbol{\nu}_{i}}{\sqrt{T}}\right)\right]
\end{aligned}
$$


For a fixed $n$, because $\frac{1}{T \sqrt{T}} \mathbf{x}_{i}^{\prime} \mathbf{A}^{-1} \iota_{T} \Rightarrow(1-\rho)^{2} \varpi_{\varepsilon} \int W_{i}+o_{p}(1)$, which is proved in (a) and

$$
\begin{aligned}
& \frac{1}{T} \mathbf{x}_{i}^{\prime} \mathbf{A}^{-1} \boldsymbol{\nu}_{i} \\
= & \frac{1}{T} \sum_{t=1}^{T}\left(x_{i t}-\rho x_{i t-1}\right) e_{i t} \\
= & \frac{1}{T} \sum_{t=1}^{T}\left((1-\rho) x_{i t-1}+\varepsilon_{i t}\right) e_{i t} \\
= & (1-\rho) \frac{1}{T} \sum_{t=1}^{T}\left(x_{i t-1} e_{i t}\right)+\frac{1}{T} \sum_{t=1}^{T}\left(\varepsilon_{i t} e_{i t}\right) \\
\Rightarrow & (1-\rho)\left[\varpi_{\varepsilon} \varpi_{e . \varepsilon}^{1 / 2}\left(\int W_{i} d V_{i}\right)+\varpi_{\varepsilon e}\left(\int W_{i} d W_{i}\right)+\gamma_{\varepsilon e}\right]+\sigma_{\varepsilon e},
\end{aligned}
$$

and

$$
\frac{1}{\sqrt{T}} \boldsymbol{\iota}_{T}^{\prime} \mathbf{A}^{-1} \boldsymbol{\nu}_{i}=(1-\rho) \frac{1}{\sqrt{T}} \sum_{t=1}^{T} e_{i t} \Rightarrow(1-\rho) \varpi_{e} V_{i}(1),
$$

as $T \rightarrow \infty$. Hence,

$$
\begin{aligned}
& \frac{1}{n T} \mathbf{x}^{\prime} \Phi^{-1} \mathbf{u} \\
= & \frac{1}{\varpi_{e}^{2}} \frac{1}{n} \sum_{i=1}^{n}\left[\frac{1}{\sqrt{T}}\left(\frac{\varpi_{e}^{2} \mu_{i} T}{\varpi_{e}^{2}+\theta \sigma_{\mu}^{2}} \frac{\mathbf{x}_{i}^{\prime} \mathbf{A}^{-1} \boldsymbol{\iota}_{T}}{T \sqrt{T}}\right)+\left(\frac{\mathbf{x}_{i}^{\prime} \mathbf{A}^{-1} \boldsymbol{\nu}_{i}}{T}-\frac{\sigma_{\mu}^{2} T}{\varpi_{e}^{2}+\theta \sigma_{\mu}^{2}} \frac{\mathbf{x}_{i}^{\prime} \mathbf{A}^{-1} \boldsymbol{\iota}_{T}}{T \sqrt{T}} \frac{\boldsymbol{\iota}_{T}^{\prime} \mathbf{A}^{-1} \boldsymbol{\nu}_{i}}{\sqrt{T}}\right)\right] \\
\Rightarrow & \frac{1}{\varpi_{e}^{2}} \frac{1}{n} \sum_{i=1}^{n}\left\{(1-\rho)\left[\varpi_{\varepsilon} \varpi_{e . \varepsilon}^{1 / 2}\left(\int W_{i} d V_{i}\right)+\varpi_{\varepsilon e}\left(\int W_{i} d W_{i}\right)+\gamma_{\varepsilon e}\right]+\sigma_{\varepsilon e}\right. \\
& \left.-\frac{1}{(1-\rho)^{2}}(1-\rho)^{3}\left[\varpi_{\varepsilon} \varpi_{e . \varepsilon}^{1 / 2}\left(\int W_{i}\right) V_{i}(1)+\varpi_{\varepsilon e}\left(\int W_{i}\right) W_{i}(1)\right]\right\}+o_{p}(1) \\
= & \frac{1}{\varpi_{e}^{2}} \frac{1}{n} \sum_{i=1}^{n}\left\{(1-\rho)\left[\varpi_{\varepsilon} \varpi_{e . \varepsilon}^{1 / 2}\left(\int \tilde{W}_{i} d V_{i}\right)+\varpi_{\varepsilon e}\left(\iint_{i} \tilde{W} d W_{i}\right)+\gamma_{\varepsilon e}\right]+\sigma_{\varepsilon e}\right\} \\
& \frac{p}{\rightarrow} \frac{1}{\varpi_{e}^{2}}\left[(1-\rho)\left[-\frac{1}{2} \varpi_{\varepsilon e}+\gamma_{\varepsilon e}\right]+\sigma_{\varepsilon e}\right]
\end{aligned}
$$

as $n \rightarrow \infty$. 
And because

$$
\begin{aligned}
\frac{1}{n \sqrt{T}} \iota_{n T}^{\prime} \Phi^{-1} \mathbf{u} & =\frac{1}{n} \sum_{i=1}^{n} \frac{1}{\sqrt{T}} \frac{\theta}{\varpi_{e}^{2}+\theta \sigma_{\mu}^{2}}\left(\mu_{i}+\boldsymbol{\iota}_{T}^{\prime} \mathbf{A}^{-1} \boldsymbol{\nu}_{i}\right) \\
& =\frac{1}{\sqrt{T}} \frac{1}{n} \sum_{i=1}^{n}\left[\left(\frac{\theta}{\varpi_{e}^{2}+\theta \sigma_{\mu}^{2}}\right) \mu_{i}\right]+\frac{1}{n} \sum_{i=1}^{n}\left[\frac{\theta}{\varpi_{e}^{2}+\theta \sigma_{\mu}^{2}}\left(\frac{\boldsymbol{\iota}_{T}^{\prime} \mathbf{A}^{-1} \boldsymbol{\nu}_{i}}{\sqrt{T}}\right)\right] \\
& \Rightarrow o_{p}(1)+\frac{1}{n} \sum_{i=1}^{n}\left[\frac{(1-\rho) \varpi_{e}}{\sigma_{\mu}^{2}} V_{i}(1)\right] \\
& \stackrel{p}{\rightarrow} 0
\end{aligned}
$$

and also because $\frac{1}{n \sqrt{T}} \mathbf{x}^{\prime} \Phi^{-1} \boldsymbol{\iota}_{n T} \stackrel{p}{\rightarrow} 0$ and $\frac{1}{n} \iota_{N T}^{\prime} \Phi^{-1} \boldsymbol{\iota}_{n T} \stackrel{p}{\rightarrow} \frac{1}{\sigma_{\mu}^{2}}$, which are proved in part (a). Hence, we have

$$
\begin{aligned}
\frac{1}{n T} G_{2}= & \frac{1}{n T} \mathbf{x}^{\prime} \Phi^{-1} \mathbf{u}-\frac{\mathbf{x}^{\prime} \Phi^{-1} \iota_{n T}}{n \sqrt{T}}\left(\frac{\boldsymbol{\iota}_{n T}^{\prime} \Phi^{-1} \iota_{n T}}{n}\right)^{-1} \frac{\boldsymbol{\iota}_{n T}^{\prime} \Phi^{-1} \mathbf{u}}{n \sqrt{T}} \\
& \stackrel{p}{\rightarrow} \frac{1}{\varpi_{e}^{2}}\left[(1-\rho)\left[-\frac{1}{2} \varpi_{\varepsilon e}+\gamma_{\varepsilon e}\right]+\sigma_{\varepsilon e}\right]
\end{aligned}
$$

Note that

$$
\frac{1}{\sqrt{n} T} G_{2}-\frac{1}{\varpi_{e}^{2}} \frac{1}{\sqrt{n}} \sum_{i=1}^{n}(1-\rho)\left[\left(\frac{1}{T} \sum_{t=1}^{T}\left(x_{i t}-\bar{x}_{i}\right) \varepsilon_{i t}\right) \frac{\varpi_{\varepsilon e}}{\varpi_{\varepsilon}^{2}}+\gamma_{\varepsilon e}+\frac{\sigma_{\varepsilon e}}{1-\rho}\right]
$$

First consider that

$$
\begin{aligned}
& \frac{1}{\sqrt{n} T} \mathbf{x}^{\prime} \Phi^{-1} \mathbf{u} \\
= & \frac{1}{\varpi_{e}^{2}} \frac{1}{\sqrt{n}} \sum_{i=1}^{n}\left[\frac{1}{\sqrt{T}}\left(\frac{\varpi_{e}^{2} \mu_{i} T}{\varpi_{e}^{2}+\theta \sigma_{\mu}^{2}} \frac{\mathbf{x}_{i}^{\prime} \mathbf{A}^{-1} \iota_{T}}{T \sqrt{T}}\right)+\left(\frac{\mathbf{x}_{i}^{\prime} \mathbf{A}^{-1} \boldsymbol{\nu}_{i}}{T}-\frac{\sigma_{\mu}^{2} T}{\varpi_{e}^{2}+\theta \sigma_{\mu}^{2}} \frac{1}{T^{2}} \mathbf{x}_{i}^{\prime} \mathbf{A}^{-1} \iota_{T} \boldsymbol{\iota}_{T}^{\prime} \mathbf{A}^{-1} \boldsymbol{\nu}_{i}\right)\right]
\end{aligned}
$$

For a fixed $n$, because $\frac{1}{T \sqrt{T}} \mathbf{x}_{i}^{\prime} \mathbf{A}^{-1} \iota_{T} \Rightarrow(1-\rho)^{2} \varpi_{\varepsilon} \int W_{i}+o_{p}(1)$, which is proved 
in (a) and

$$
\begin{aligned}
& \frac{1}{T^{2}} \mathbf{x}_{i}^{\prime} \mathbf{A}^{-1} \iota_{T} \iota_{T}^{\prime} \mathbf{A}^{-1} \boldsymbol{\nu}_{i} \\
= & \frac{1}{T^{2}}\left[(1-\rho) \sum_{t=1}^{T}\left(x_{i t}-\rho x_{i t-1}\right)\right]\left[(1-\rho) \sum_{t=1}^{T} e_{i t}\right] \\
= & (1-\rho)^{2} \frac{1}{T^{2}}\left[\sum_{t=1}^{T}\left((1-\rho) x_{i t-1}+\varepsilon_{i t}\right)\right]\left[\sum_{t=1}^{T} e_{i t}\right] \\
= & (1-\rho)^{3}\left[\frac{1}{T \sqrt{T}} \sum_{t=1}^{T} x_{i t-1}\right]\left[\frac{1}{\sqrt{T}} \sum_{t=1}^{T} e_{i t}\right]+(1-\rho)^{2} \frac{1}{\sqrt{T}}\left[\frac{1}{\sqrt{T}} \sum_{t=1}^{T} \varepsilon_{i t}\right]\left[\frac{1}{T} \sum_{t=1}^{T} e_{i t}\right] \\
\Rightarrow & (1-\rho)^{3}\left[\varpi_{\varepsilon} \varpi_{e . \varepsilon}^{1 / 2}\left(\int W_{i}\right) V_{i}(1)+\varpi_{\varepsilon e}\left(\int W_{i}\right) W_{i}(1)\right]+\frac{1}{\sqrt{T}} o_{p}(1)
\end{aligned}
$$

as $T \rightarrow \infty$. Hence,

$$
\begin{aligned}
& \frac{1}{\sqrt{n} T} \mathbf{x}^{\prime} \Phi^{-1} \mathbf{u}-\frac{1}{\varpi_{e}^{2}} \frac{1}{\sqrt{n}} \sum_{i=1}^{n}(1-\rho)\left[\left(\frac{1}{T} \sum_{t=1}^{T}\left(x_{i t}-\bar{x}_{i}\right) \varepsilon_{i t}\right) \frac{\varpi_{\varepsilon e}}{\varpi_{\varepsilon}^{2}}+\gamma_{\varepsilon e}+\frac{\sigma_{\varepsilon e}}{1-\rho}\right] \\
\Rightarrow & \frac{1}{\varpi_{e}^{2}} \frac{1}{\sqrt{n}} \sum_{i=1}^{n}\left[(1-\rho)\left[\varpi_{\varepsilon} \varpi_{e . \varepsilon}^{1 / 2}\left(\int_{i} \tilde{W} d V_{i}\right)+\varpi_{\varepsilon e}\left(\int_{i} \tilde{W} d W_{i}\right)\right]+\sigma_{\varepsilon e}\right] \\
& -\frac{1}{\varpi_{e}^{2}} \frac{1}{\sqrt{n}} \sum_{i=1}^{n}(1-\rho)\left[\varpi_{\varepsilon e}\left(\int_{i} \tilde{W} d W_{i}\right)+\gamma_{\varepsilon e}+\frac{\sigma_{\varepsilon e}}{1-\rho}\right] \\
= & \frac{1-\rho}{\varpi_{e}^{2}} \frac{1}{\sqrt{n}} \sum_{i=1}^{n}\left[\varpi_{\varepsilon} \varpi_{e . \varepsilon}^{1 / 2}\left(\int_{i} \tilde{W} d V_{i}\right)\right] \\
\Rightarrow & N\left(0, \frac{(1-\rho)^{2} \varpi_{\varepsilon}^{2} \varpi_{e . \varepsilon}}{6 \varpi_{e}^{4}}\right)
\end{aligned}
$$

as $n \rightarrow \infty$.

And because

$$
\begin{aligned}
\frac{1}{\sqrt{n T}} \boldsymbol{\iota}_{n T}^{\prime} \Phi^{-1} \mathbf{u} & =\frac{1}{\sqrt{n}} \sum_{i=1}^{n} \frac{1}{\sqrt{T}} \frac{\theta}{\varpi_{e}^{2}+\theta \sigma_{\mu}^{2}}\left(\mu_{i}+\boldsymbol{\iota}_{T}^{\prime} \mathbf{A}^{-1} \boldsymbol{\nu}_{i}\right) \\
& =\frac{1}{\sqrt{T}} \frac{1}{\sqrt{n}} \sum_{i=1}^{n} \frac{\theta}{\varpi_{e}^{2}+\theta \sigma_{\mu}^{2}} \mu_{i}+\frac{1}{\sqrt{n}} \sum_{i=1}^{n} \frac{\theta}{\varpi_{e}^{2}+\theta \sigma_{\mu}^{2}} \frac{\boldsymbol{\iota}_{T}^{\prime} \mathbf{A}^{-1} \boldsymbol{\nu}_{i}}{\sqrt{T}} \\
& \Rightarrow o_{p}(1)+\frac{1}{\sqrt{n}} \sum_{i=1}^{n}\left[\frac{(1-\rho) \varpi_{e}}{\sigma_{\mu}^{2}} V_{i}(1)\right] \\
& \Rightarrow \frac{(1-\rho) \varpi_{e}}{\sigma_{\mu}^{2}} N(0,1)
\end{aligned}
$$


and also because $\frac{1}{n} \mathbf{x}^{\prime} \Phi^{-1} \iota_{n T} \stackrel{p}{\rightarrow} 0$ and $\frac{1}{n} \iota_{N T}^{\prime} \Phi^{-1} \iota_{n T} \stackrel{p}{\rightarrow} \frac{1}{\sigma_{\mu}^{2}}$, which are proved in part (a). Hence, we have

$$
\begin{aligned}
& \frac{1}{\sqrt{n} T} G_{2}-\frac{1}{\varpi_{e}^{2}} \frac{1}{\sqrt{n}} \sum_{i=1}^{n}(1-\rho)\left[\left(\frac{1}{T} \sum_{t=1}^{T}\left(x_{i t}-\bar{x}_{i}\right) \varepsilon_{i t}\right) \frac{\varpi_{\varepsilon e}}{\varpi_{\varepsilon}^{2}}+\gamma_{\varepsilon e}+\frac{\sigma_{\varepsilon e}}{1-\rho}\right] \\
= & \frac{1}{\sqrt{n} T} \mathbf{x}^{\prime} \Phi^{-1} \mathbf{u}-\frac{1}{\varpi_{e}^{2}} \frac{1}{\sqrt{n}} \sum_{i=1}^{n}(1-\rho)\left[\left(\frac{1}{T} \sum_{t=1}^{T}\left(x_{i t}-\bar{x}_{i}\right) \varepsilon_{i t}\right) \frac{\varpi_{\varepsilon e}}{\varpi_{\varepsilon}^{2}}+\gamma_{\varepsilon e}+\frac{\sigma_{\varepsilon e}}{1-\rho}\right] \\
& -\frac{\mathbf{x}^{\prime} \Phi^{-1} \boldsymbol{\iota}_{n T}}{n \sqrt{T}}\left(\frac{\boldsymbol{\iota}_{n T}^{\prime} \Phi^{-1} \iota_{n T}}{n}\right)^{-1} \frac{\boldsymbol{\iota}_{n T}^{\prime} \Phi^{-1} \mathbf{u}}{\sqrt{n T}} \\
\Rightarrow & N\left(0, \frac{(1-\rho)^{2} \varpi_{\varepsilon}^{2} \varpi_{e . \varepsilon}}{6 \varpi_{e}^{4}}\right) .
\end{aligned}
$$

Note that

$$
\frac{1}{n T} G_{1}=\frac{1}{n T} \mathbf{x}^{\prime} \Phi^{-1} \mathbf{x}-\frac{1}{T} \frac{\mathbf{x}^{\prime} \Phi^{-1} \iota_{n T}}{n}\left(\frac{\boldsymbol{\iota}_{n T}^{\prime} \Phi^{-1} \iota_{n T}}{n}\right)^{-1} \frac{\boldsymbol{\iota}_{n T}^{\prime} \Phi^{-1} \mathbf{x}}{n}
$$

First consider that

$$
\begin{aligned}
& \frac{1}{n T} \mathbf{x}^{\prime} \Phi^{-1} \mathbf{x} \\
= & \frac{1}{\sigma_{e}^{2}} \frac{1}{n} \sum_{i=1}^{n}\left(\frac{1}{T} \mathbf{x}_{i}^{\prime} \mathbf{A}^{-1} \mathbf{x}_{i}-\frac{\sigma_{\mu}^{2}}{\sigma_{e}^{2}+\theta \sigma_{\mu}^{2}} \frac{\mathbf{x}_{i}^{\prime} \mathbf{A}^{-1} \iota_{T}}{\sqrt{T}} \frac{\boldsymbol{\iota}_{T}^{\prime} \mathbf{A}^{-1} \mathbf{x}_{i}}{\sqrt{T}}\right)
\end{aligned}
$$

For a fixed $n$, because

$$
\begin{aligned}
& \frac{1}{T} \mathbf{x}_{i}^{\prime} \mathbf{A}^{-1} \mathbf{x}_{i} \\
= & \frac{1}{T} \sum_{t=1}^{T}\left(x_{i t}-x_{i t-1}\right)^{2} \\
= & \frac{1}{T} \sum_{t=1}^{T} \varepsilon_{i t}^{2} \\
\Rightarrow & \sigma_{\varepsilon}^{2},
\end{aligned}
$$

and

$$
\frac{1}{\sqrt{T}} \mathbf{x}_{i}^{\prime} \mathbf{A}^{-1} \iota_{T}=\frac{1}{\sqrt{T}} x_{i 1} \stackrel{p}{\rightarrow} 0
$$


as $T \rightarrow \infty$. Hence,

$$
\begin{aligned}
& \frac{1}{n T} \mathbf{x}^{\prime} \Phi^{-1} \mathbf{x} \\
= & \frac{1}{\sigma_{e}^{2}} \frac{1}{n} \sum_{i=1}^{n}\left(\frac{1}{T} \mathbf{x}_{i}^{\prime} \mathbf{A}^{-1} \mathbf{x}_{i}-\frac{\sigma_{\mu}^{2}}{\sigma_{e}^{2}+\theta \sigma_{\mu}^{2}} \frac{\mathbf{x}_{i}^{\prime} \mathbf{A}^{-1} \iota_{T}}{\sqrt{T}} \frac{\boldsymbol{\iota}_{T}^{\prime} \mathbf{A}^{-1} \mathbf{x}_{i}}{\sqrt{T}}\right) \\
& \stackrel{p}{\rightarrow} \frac{\sigma_{\varepsilon}^{2}}{\varpi_{e}^{2}} .
\end{aligned}
$$

as $n \rightarrow \infty$.

Also because

$$
\begin{aligned}
\frac{1}{n} \mathbf{x}^{\prime} \Phi^{-1} \iota_{n T}= & \frac{1}{n} \sum_{i=1}^{n} \frac{1}{\varpi_{e}^{2}+\theta \sigma_{\mu}^{2}}\left(\mathbf{x}_{i}^{\prime} \mathbf{A}^{-1} \iota_{T}\right) \\
\Rightarrow & \frac{1}{n} \sum_{i=1}^{n} \frac{1}{\varpi_{e}^{2}+\sigma_{\mu}^{2}} x_{i 1} \\
& \stackrel{p}{\rightarrow} 0
\end{aligned}
$$

because $\theta=1$. And

$$
\frac{1}{n} \iota_{n T}^{\prime} \Phi^{-1} \iota_{n T}=\frac{1}{n} \frac{n \theta}{\varpi_{e}^{2}+\theta \sigma_{\mu}^{2}} \stackrel{p}{\rightarrow} \frac{1}{\sigma_{\mu}^{2}}
$$

Hence, we have

$$
\begin{aligned}
\frac{1}{n T} G_{1}= & \frac{1}{n T} \mathbf{x}^{\prime} \Phi^{-1} \mathbf{x}-\frac{1}{T} \frac{\mathbf{x}^{\prime} \Phi^{-1} \boldsymbol{\iota}_{n T}}{n}\left(\frac{\boldsymbol{\iota}_{n T}^{\prime} \Phi^{-1} \iota_{n T}}{n}\right)^{-1} \frac{\boldsymbol{\iota}_{n T}^{\prime} \Phi^{-1} \mathbf{x}}{n} \\
& \stackrel{p}{\rightarrow} \frac{\sigma_{\varepsilon}^{2}}{\varpi_{e}^{2}}
\end{aligned}
$$

Note that

$$
\frac{1}{n T} G_{2}=\frac{1}{n T} \mathbf{x}^{\prime} \Phi^{-1} \mathbf{u}-\frac{1}{T} \frac{\mathbf{x}^{\prime} \Phi^{-1} \boldsymbol{\iota}_{n T}}{n}\left(\frac{\boldsymbol{\iota}_{n T}^{\prime} \Phi^{-1} \boldsymbol{\iota}_{n T}}{n}\right)^{-1} \frac{\boldsymbol{\iota}_{n T}^{\prime} \Phi^{-1} \mathbf{u}}{n}
$$

First consider that

$$
\begin{aligned}
& \frac{1}{n T} \mathbf{x}^{\prime} \Phi^{-1} \mathbf{u} \\
= & \frac{1}{\varpi_{e}^{2}} \frac{1}{n} \sum_{i=1}^{n}\left[\frac{1}{\sqrt{T}} \frac{\varpi_{e}^{2} \mu_{i}}{\varpi_{e}^{2}+\theta \sigma_{\mu}^{2}} \frac{\mathbf{x}_{i}^{\prime} \mathbf{A}^{-1} \iota_{T}}{\sqrt{T}}+\frac{1}{T} \mathbf{x}_{i}^{\prime} \mathbf{A}^{-1} \boldsymbol{\nu}_{i}-\frac{1}{\sqrt{T}} \frac{\sigma_{\mu}^{2}}{\varpi_{e}^{2}+\theta \sigma_{\mu}^{2}} \frac{\mathbf{x}_{i}^{\prime} \mathbf{A}^{-1} \iota_{T} \boldsymbol{\iota}_{T}^{\prime} \mathbf{A}^{-1} \boldsymbol{\nu}_{i}}{\sqrt{T}}\right]
\end{aligned}
$$

For a fixed $n$, because $\frac{1}{\sqrt{T}} \mathbf{x}_{i}^{\prime} \mathbf{A}^{-1} \iota_{T}=\frac{1}{\sqrt{T}} x_{i 1} \stackrel{p}{\rightarrow} 0$, which is proved in part (a) and

$$
\frac{1}{T} \mathbf{x}_{i}^{\prime} \mathbf{A}^{-1} \boldsymbol{\nu}_{i}=\frac{1}{T} \sum_{t=1}^{T} \varepsilon_{i t} e_{i t} \stackrel{p}{\rightarrow} \sigma_{\varepsilon e}
$$


and

$$
\frac{1}{\sqrt{T}} \mathbf{x}_{i}^{\prime} \mathbf{A}^{-1} \iota_{T} \iota_{T}^{\prime} \mathbf{A}^{-1} \boldsymbol{\nu}_{i}=\frac{1}{\sqrt{T}} x_{i 1} \nu_{i 1}^{\prime} \stackrel{p}{\rightarrow} 0,
$$

as $T \rightarrow \infty$. Hence,

$$
\begin{aligned}
& \frac{1}{n T} \mathbf{x}^{\prime} \Phi^{-1} \mathbf{u} \\
= & \frac{1}{\varpi_{e}^{2}} \frac{1}{n} \sum_{i=1}^{n}\left[\frac{1}{\sqrt{T}} \frac{\varpi_{e}^{2} \mu_{i}}{\varpi_{e}^{2}+\theta \sigma_{\mu}^{2}} \frac{\mathbf{x}_{i}^{\prime} \mathbf{A}^{-1} \iota_{T}}{\sqrt{T}}+\frac{1}{T} \mathbf{x}_{i}^{\prime} \mathbf{A}^{-1} \boldsymbol{\nu}_{i}-\frac{1}{\sqrt{T}} \frac{\sigma_{\mu}^{2}}{\varpi_{e}^{2}+\theta \sigma_{\mu}^{2}} \frac{\mathbf{x}_{i}^{\prime} \mathbf{A}^{-1} \iota_{T} \boldsymbol{\iota}_{T}^{\prime} \mathbf{A}^{-1} \boldsymbol{\nu}_{i}}{\sqrt{T}}\right] \\
& \stackrel{p}{\rightarrow} \frac{\sigma_{\varepsilon e}}{\varpi_{e}^{2}}
\end{aligned}
$$

as $n \rightarrow \infty$.

And because

$$
\begin{aligned}
& \frac{1}{n} \iota_{n T}^{\prime} \Phi^{-1} \mathbf{u} \\
= & \frac{1}{n} \sum_{i=1}^{n} \frac{\theta}{\varpi_{e}^{2}+\theta \sigma_{\mu}^{2}}\left(\mu_{i}+\boldsymbol{\iota}_{T}^{\prime} \mathbf{A}^{-1} \boldsymbol{\nu}_{i}\right) \\
= & \frac{1}{n} \sum_{i=1}^{n} \frac{1}{\varpi_{e}^{2}+\sigma_{\mu}^{2}}\left(\mu_{i}+\nu_{i 1}^{\prime}\right) \\
& \stackrel{p}{\rightarrow} 0
\end{aligned}
$$

and also because $\frac{1}{n} \mathbf{x}^{\prime} \Phi^{-1} \iota_{n T} \stackrel{p}{\rightarrow} 0$ and $\frac{1}{n} \iota_{N T}^{\prime} \Phi^{-1} \iota_{n T} \stackrel{p}{\rightarrow} \frac{1}{\sigma_{\mu}^{2}}$, which are proved in part (a). Hence, we have

$$
\begin{aligned}
\frac{1}{n T} G_{2}= & \frac{1}{n T} \mathbf{x}^{\prime} \Phi^{-1} \mathbf{u}-\frac{1}{T} \frac{\mathbf{x}^{\prime} \Phi^{-1} \boldsymbol{\iota}_{n T}}{n}\left(\frac{\boldsymbol{\iota}_{n T}^{\prime} \Phi^{-1} \iota_{n T}}{n}\right)^{-1} \frac{\boldsymbol{\iota}_{n T}^{\prime} \Phi^{-1} \mathbf{u}}{n} \\
& \stackrel{p}{\rightarrow} \frac{\sigma_{\varepsilon e}}{\varpi_{e}^{2}}
\end{aligned}
$$

Note that

$$
\frac{1}{\sqrt{n T}} G_{2}=\frac{1}{\sqrt{n T}} \mathbf{x}^{\prime} \Phi^{-1} \mathbf{u}-\frac{1}{\sqrt{T}} \frac{\mathbf{x}^{\prime} \Phi^{-1} \boldsymbol{\iota}_{n T}}{n}\left(\frac{\boldsymbol{\iota}_{n T}^{\prime} \Phi^{-1} \iota_{n T}}{n}\right)^{-1} \frac{\boldsymbol{\iota}_{n T}^{\prime} \Phi^{-1} \mathbf{u}}{\sqrt{n}}
$$

First consider that

$$
\begin{aligned}
& \sqrt{n T}\left(\frac{1}{n T} \mathbf{x}^{\prime} \Phi^{-1} \mathbf{u}-\frac{\sigma_{\varepsilon e}}{\varpi_{e}^{2}}\right) \\
= & \frac{1}{\varpi_{e}^{2}} \frac{1}{\sqrt{n}} \sum_{i=1}^{n}\left[\frac{\varpi_{e}^{2} \mu_{i} T}{\varpi_{e}^{2}+\theta \sigma_{\mu}^{2}} \frac{\mathbf{x}_{i}^{\prime} \mathbf{A}^{-1} \iota_{T}}{\sqrt{T}}+\frac{1}{\sqrt{T}} \mathbf{x}_{i}^{\prime} \mathbf{A}^{-1} \boldsymbol{\nu}_{i}-\frac{\sigma_{\mu}^{2}}{\varpi_{e}^{2}+\theta \sigma_{\mu}^{2}} \frac{1}{\sqrt{T}} \mathbf{x}_{i}^{\prime} \mathbf{A}^{-1} \iota_{T} \boldsymbol{\iota}_{T}^{\prime} \mathbf{A}^{-1} \boldsymbol{\nu}_{i}\right]-\sqrt{n T} \frac{\sigma_{\varepsilon e}}{\varpi_{e}^{2}}
\end{aligned}
$$


For a fixed $n$, because $\frac{1}{\sqrt{T}} \mathbf{x}_{i}^{\prime} \mathbf{A}^{-1} \iota_{T}=\frac{1}{\sqrt{T}} x_{i 1} \stackrel{p}{\rightarrow} 0$, which is proved in part (a) and

$$
\begin{aligned}
& \sqrt{T}\left(\frac{1}{T} \mathbf{x}_{i}^{\prime} \mathbf{A}^{-1} \boldsymbol{\nu}_{i}-\sigma_{\varepsilon e}\right) \\
= & \frac{1}{\sqrt{T}} \sum_{t=1}^{T}\left(x_{i t}-x_{i t-1}\right) e_{i t}+o(1)-\sqrt{T} \sigma_{\varepsilon e} \\
= & \frac{1}{\sqrt{T}} \sum_{t=1}^{T} \varepsilon_{i t} e_{i t}+o(1)-\sqrt{T} \sigma_{\varepsilon e} \\
\Rightarrow & N\left(0, \varpi_{e}^{2} \varpi_{\varepsilon}^{2}\right),
\end{aligned}
$$

and $\frac{1}{\sqrt{T}} \mathbf{x}_{i}^{\prime} \mathbf{A}^{-1} \iota_{T} \boldsymbol{\iota}_{T}^{\prime} \mathbf{A}^{-1} \boldsymbol{\nu}_{i}=\frac{1}{\sqrt{T}} x_{i 1} \nu_{i 1}^{\prime} \stackrel{p}{\rightarrow} 0$, which is proved in part (a), as $T \rightarrow \infty$. Hence,

$$
\begin{aligned}
& \sqrt{n T}\left(\frac{1}{n T} \mathbf{x}^{\prime} \Phi^{-1} \mathbf{u}-\frac{\sigma_{\varepsilon e}}{\varpi_{e}^{2}}\right) \\
= & \frac{1}{\varpi_{e}^{2}} \frac{1}{\sqrt{n}} \sum_{i=1}^{n}\left[\frac{\varpi_{e}^{2} \mu_{i} T}{\varpi_{e}^{2}+\theta \sigma_{\mu}^{2}} \frac{\mathbf{x}_{i}^{\prime} \mathbf{A}^{-1} \iota_{T}}{\sqrt{T}}+\frac{1}{\sqrt{T}} \mathbf{x}_{i}^{\prime} \mathbf{A}^{-1} \boldsymbol{\nu}_{i}-\frac{\sigma_{\mu}^{2}}{\varpi_{e}^{2}+\theta \sigma_{\mu}^{2}} \frac{1}{\sqrt{T}} \mathbf{x}_{i}^{\prime} \mathbf{A}^{-1} \iota_{T} \boldsymbol{\iota}_{T}^{\prime} \mathbf{A}^{-1} \boldsymbol{\nu}_{i}\right]-\sqrt{n T} \frac{\sigma_{\varepsilon e}}{\varpi_{e}^{2}} \\
\Rightarrow & \frac{1}{\varpi_{e}^{2}} \frac{1}{\sqrt{n}} \sum_{i=1}^{n}\left[o_{p}(1)+N\left(0, \varpi_{e}^{2} \varpi_{\varepsilon}^{2}\right)-o_{p}(1)\right] \\
\Rightarrow & \frac{1}{\varpi_{e}^{2}} N\left(0, \varpi_{e}^{2} \varpi_{\varepsilon}^{2}\right)
\end{aligned}
$$

as $n \rightarrow \infty$.

Also because

$$
\begin{aligned}
& \frac{1}{\sqrt{n}} \boldsymbol{\iota}_{n T}^{\prime} \Phi^{-1} \mathbf{u} \\
= & \frac{1}{\sqrt{n}} \sum_{i=1}^{n} \frac{\theta}{\varpi_{e}^{2}+\theta \sigma_{\mu}^{2}}\left(\mu_{i}+\boldsymbol{\iota}_{T}^{\prime} \mathbf{A}^{-1} \boldsymbol{\nu}_{i}\right) \\
\Rightarrow & \frac{1}{\sqrt{n}} \sum_{i=1}^{n} \frac{1}{\varpi_{e}^{2}+\sigma_{\mu}^{2}}\left(\mu_{i}+\nu_{i 1}^{\prime}\right) \\
\Rightarrow & \frac{\varpi_{e}+\sigma_{\mu}}{\sigma_{\mu}^{2}} N(0,1)
\end{aligned}
$$

and also because $\frac{1}{n} \mathbf{x}^{\prime} \Phi^{-1} \boldsymbol{\iota}_{n T} \stackrel{p}{\rightarrow} 0$ and $\frac{1}{n} \iota_{N T}^{\prime} \Phi^{-1} \boldsymbol{\iota}_{n T} \stackrel{p}{\rightarrow} \frac{1}{\sigma_{\mu}^{2}}$, which are proved in 
part (a). Hence, we have

$$
\begin{aligned}
& \frac{1}{\sqrt{n T}} G_{2}-\sqrt{n T} \frac{\sigma_{\varepsilon e}}{\varpi_{e}^{2}} \\
= & {\left[\frac{1}{\sqrt{n T}} \mathbf{x}^{\prime} \Phi^{-1} \mathbf{u}-\sqrt{n T} \frac{\sigma_{\varepsilon e}}{\varpi_{e}^{2}}\right] } \\
& -\frac{1}{\sqrt{T}} \frac{\mathbf{x}^{\prime} \Phi^{-1} \iota_{n T}}{n}\left(\frac{\boldsymbol{\iota}_{n T}^{\prime} \Phi^{-1} \iota_{n T}}{n}\right)^{-1} \frac{\boldsymbol{\iota}_{n T}^{\prime} \Phi^{-1} \mathbf{u}}{\sqrt{n}} \\
\Rightarrow & \frac{1}{\varpi_{e}^{2}} N\left(0, \varpi_{e}^{2} \varpi_{\varepsilon}^{2}\right)
\end{aligned}
$$

\section{Proof of Theorem 4:}

Proof. By $\widehat{\beta}_{G L S}-\beta=G_{1}^{-1} G_{2}=\left[\mathbf{x}^{\prime} \Phi^{-1} \mathbf{x}-\mathbf{x}^{\prime} \Phi^{-1} \iota_{n T}\left(\iota_{N T}^{\prime} \Phi^{-1} \iota_{n T}\right)^{-1} \iota_{N T}^{\prime} \Phi^{-1} \mathbf{x}\right]^{-1}$ $\left[\mathbf{x}^{\prime} \Phi^{-1} \mathbf{u}-\mathbf{x}^{\prime} \Phi^{-1} \iota_{n T}\left(\iota_{N T}^{\prime} \Phi^{-1} \iota_{n T}\right)^{-1} \boldsymbol{\iota}_{N T}^{\prime} \Phi^{-1} \mathbf{u}\right]$, the proof of Theorem 4 is straightforward with above lemmas. 